NIST Measurement Services:

AT'L INST OF STAND \& TECH R.TC.

$A 11105 \quad 651034$

NIST

REFERENCE PUBLICATIONS

Spectroradıornetric Detector Measurements:

Part III-Infrared Detectors

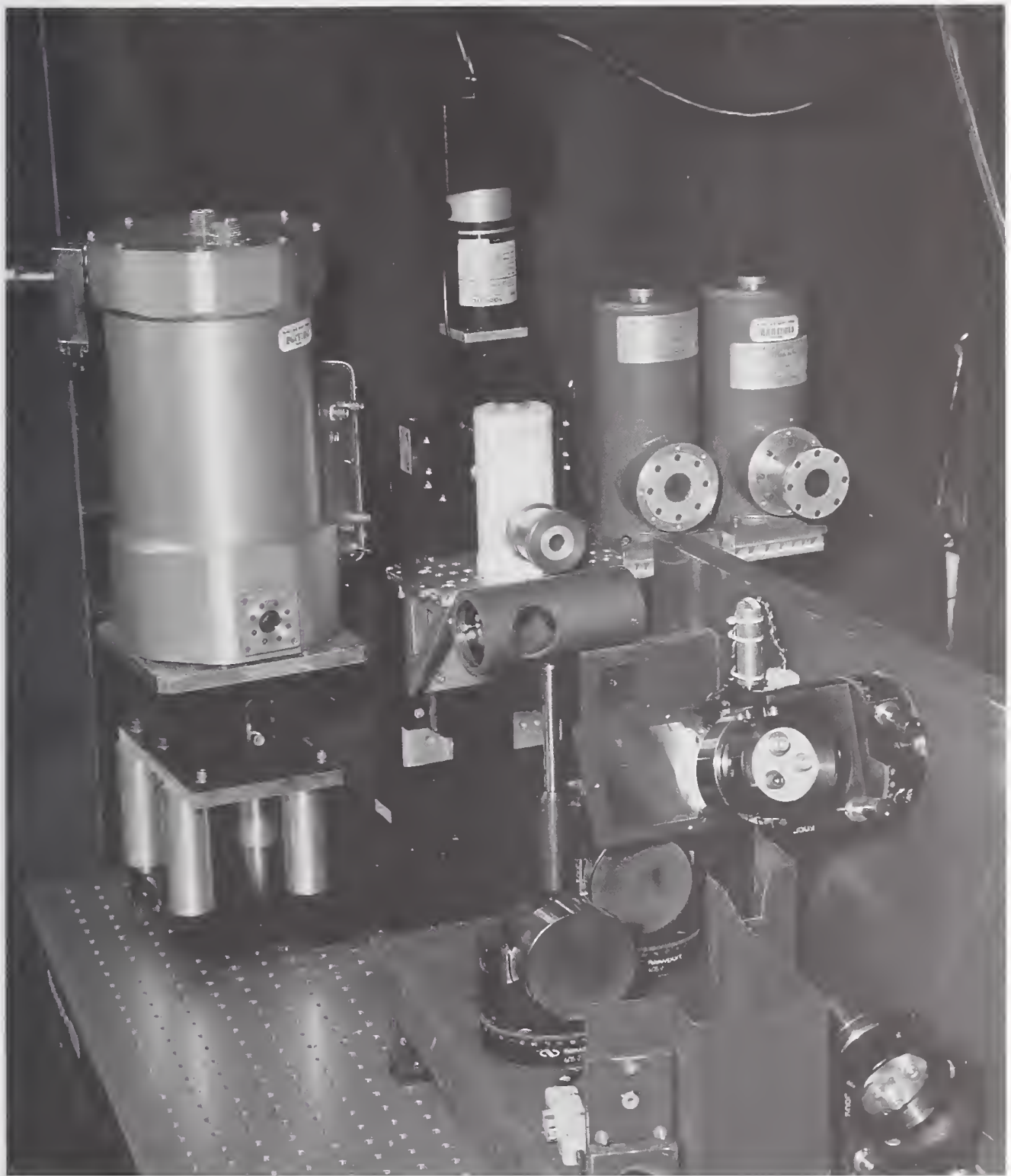

NIST

Special

Publication

$250-42$

Alan L. Migdall and George P. Eppeldauer

U.S. Department of Commerce

Technology Administration

National Institute of Standards and Technology

0(

56

$.250-42$

98 
he National Institute of Standards and Technology was established in 1988 by Congress to "assist industry in

the development of technology ... needed to improve product quality, to modernize manufacturing processes, to ensure product reliability ... and to facilitate rapid commercialization . . . of products based on new scientific discoveries."

NIST, originally founded as the National Bureau of Standards in 1901, works to strengthen U.S. industry's competitiveness; advance science and engineering; and improve public health, safety, and the environment. One of the agency's basic functions is to develop, maintain, and retain custody of the national standards of measurement, and provide the means and methods for comparing standards used in science, engineering, manufacturing, commerce, industry, and education with the standards adopted or recognized by the Federal Government.

As an agency of the U.S. Commerce Department's Technology Administration, NIST conducts basic and applied research in the physical sciences and engineering, and develops measurement techniques, test methods, standards, and related services. The Institute does generic and precompetitive work on new and advanced technologies. NIST's research facilities are located at Gaithersburg, MD 20899, and at Boulder, CO 80303. Major technical operating units and their principal activities are listed below. For more information contact the Publications and Program Inquiries Desk, 301-975-3058.

\section{Office of the Director}

- National Quality Program

- International and Academic Affairs

\section{Technology Services}

- Standards Services

- Technology Partnerships

- Measurement Services

- Technology Innovation

- Information Services

\section{Advanced Technology Program}

- Economic Assessment

- Information Technology and Applications

- Chemical and Biomedical Technology

- Materials and Manufacturing Technology

- Electronics and Photonics Technology

\section{Manufacturing Extension Partnership Program \\ - Regional Programs \\ - National Programs \\ - Program Development}

\section{Electronics and Electrical Engineering Laboratory \\ - Microelectronics \\ - Law Enforcement Standards \\ - Electricity \\ - Semiconductor Electronics \\ - Electromagnetic Fields ${ }^{1}$ \\ - Electromagnetic Technology ${ }^{1}$ \\ - Optoelectronics ${ }^{1}$}

\section{Chemical Science and Technology Laboratory \\ - Biotechnology \\ - Physical and Chemical Properties ${ }^{2}$ \\ - Analytical Chemistry \\ - Process Measurements \\ - Surface and Microanalysis Science}

Physics Laboratory

- Electron and Optical Physics

- Atomic Physics

- Optical Technology

- Ionizing Radiation

- Time and Frequency ${ }^{1}$

- Quantum Physics ${ }^{1}$

\section{Materials Science and Engineering} Laboratory

- Intelligent Processing of Materials

- Ceramics

- Materials Reliability ${ }^{1}$

- Polymers

- Metallurgy

- NIST Center for Neutron Research

\section{Manufacturing Engineering} Laboratory

- Precision Engineering

- Automated Production Technology

- Intelligent Systems

- Fabrication Technology

- Manufacturing Systems Integration

\section{Building and Fire Research} Laboratory

- Structures

- Building Materials

- Building Environment

- Fire Safety Engineering

- Fire Science

Information Technology Laboratory

- Mathematical and Computational Sciences ${ }^{2}$

- Advanced Network Technologies

- Computer Security

- Information Access and User Interfaces

- High Performance Systems and Services

- Distributed Computing and Information Services

- Software Diagnostics and Conformance Testing 


\section{NIST MEASUREMENT SERVICES:}

Spectroradiometric Detector Measurements:

Part III-Infrared Detectors

Alan L. Migdall and George P. Eppeldauer

Optical Technology Division

Physics Laboratory

National Institute of Standards and Technology

Gaithersburg, MD 20899-8400

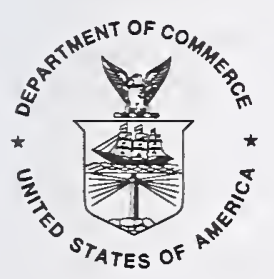

U.S. DEPARTMENT OF COMMERCE

William M. Daley, Secretary

Technology Administration

Gary R. Bachula, Acting Under Secretary for Technology

National Institute of Standards and Technology

Raymond G. Kammer, Director 
National Institute of Standards and Technology Special Publication 250-42 Natl. Inst. Stand. Technol. spec. Publ. 250-42, 59 pages (Dec. 1998) CODEN: NSPUE2

\section{U.S. GOVERNMENT PRINTING OFFICE}

WASHINGTON: 1998 


\section{Preface}

The calibration and related measurement services of the National Institute of Standards and Technology (NIST) are intended to assist the makers and users of precision measuring instruments in achieving the highest possible levels of accuracy, quality, and productivity. NIST offers over 300 different calibrations, special tests, and measurement assurance services. These services allow customers to directly link their measurement systems to measurement systems and standards maintained by NIST. These services are offered to the public and private organizations alike. They are described in NIST Special Publication (SP) 250, NIST Calibration Services Users Guide.

The Users Guide is supplemented by a number of Special Publications (designated as the "SP 250 Series") that provide detailed descriptions of the important features of specific NIST calibration services. These documents provide a description of the: (1) specifications for the services; (2) design philosophy and theory; (3) NIST measurement system; (4) NIST operational procedures; (5) assessment of the measurement uncertainty including random and systematic errors and an error budget; and (6) internal quality control procedures used by NIST. These documents will present more detail than can be given in NIST calibration reports, or than is generally allowed in articles in scientific journals. In the past, NIST has published such information in a variety of ways. This series will make this type of information more readily available to the user.

This document, SP 250-42 (1998), NIST Measurement Services: Spectroradiometric Detector Measurements: Part III--Infrared Detectors, is an extension of SP 250-41 (1998). It covers the calibration of IR photodetector absolute spectral responsivity from $1.8 \mu \mathrm{m}$ to $20 \mu \mathrm{m}$ (Service ID number 39090 S in SP 250, NIST Calibration Services Users Guide). Inquiries concerning the technical content of this document or the specifications for these services should be directed to one of the authors or to one of the technical contacts.

NIST welcomes suggestions on how publications such as this might be made more useful. Suggestions are also welcome concerning the need for new calibration services, special tests, and measurement assurance programs.

Peter L. M. Heydemann (Acting)

Director

Measurement Services
Katharine B. Gebbie

Director

Physics Laboratory 


\section{Abstract and Key Words}

The National Institute of Standards and Technology (NIST) supplies calibrations of IR photodetector's spectral radiant power responsivity from $1.8 \mu \mathrm{m}$ to $20 \mu \mathrm{m}$. The spectral responsivity of a detector under test is determined by comparison to an absolute detector standard. That absolute detector standard is a cryogenic composite Si bolometer. The spectral radiant power responsivity of the bolometer is based on detector measurements traceable to the NIST High Accuracy Cryogenic Radiometer (HACR) and on material transmittance and reflectance measurements. Silicon and Germanium photodetectors and pyroelectric detectors are used to transfer radiant power responsivity from the HACR to the bolometer over a range of wavelengths. Reflectance and transmittance measurements of the bolometer absorber and window are used to determine the continuous relative spectral responsivity of the bolometer. This relative responsivity determination combined with the absolute responsivity measurements allows a continuous absolute spectral responsivity of the bolometer to be realized. The comparison between the detector under test and the bolometer is performed using a monochromator-based facility. The detector scale relative expanded uncertainty $(k=2)$ is typically $1.6 \%$ throughout the spectral range. The uncertainty of a responsivity calibration of a specfic detector will generally be the uncertainties associated with that detector summed in quadrature with the $1.6 \%$ of the detector scale uncertainty. A description is given of the procedures, equipment, and techniques used to perform these calibrations. Detailed estimates and procedures for determining uncertainties of the reported values are also presented.

Key Words: absolute power responsivity, spectral radiant power responsivity; bolometer; calibration; cryogenic radiometer; detector; infrared; infrared detectors; measurement; optical power; photodetector; pyroelectric detector; quality system; scale; silicon photodiode; germanium photodiode, indium antimonide photodiode. 


\section{TABLE OF CONTENTS}

1. INTRODUCTION

2. NIST SPECTRORADIOMETRIC DETECTOR MEASUREMENT SERVICE

$\begin{array}{ll}\text { 2.1 Description of Measurement Services } & 3\end{array}$

2.2 Measurement Limitations 3

2.3 How To Order Special Tests 4

2.4 Technical Contacts 4

3. MEASUREMENT THEORY 5

3.1 Substitution Method 5

3.1.1 General Substitution Method 5

3.1.2 Detector Substitution 6

\begin{tabular}{ll}
3.2 Measurement Equation & 6 \\
\hline &
\end{tabular}

3.2.1 Approximations $\quad 7$

4. EQUIPMENT DESCRIPTION 8

4.1 Infrared Comparator Description $\quad 8$

4.1.1 Comparator Facility Description Overview $\quad 8$

4.1.2 Description of Sources 9

4.1.2.1 Ar Maxi-arc 9

4.1.2.2 Quartz Lamp 10

$\begin{array}{ll}\text { 4.1.2.3 Safety considerations } & 10\end{array}$

$\begin{array}{ll}\text { 4.1.3 Monochromator } & 10\end{array}$

4.1.4 Optical Relay System and Associated Hardware $\quad 11$

4.1.4.1 Translation Stages - Detector Positioning 11

4.1.4.2 Detector Alignment 11

4.1.4.3 Alignment Lasers 11

4.1.4.4 Enclosure 11

4.1.5 Transfer and Working Standards $\quad 12$

4.1.5.1 Cryogenic Bolometer Standard $\quad 12$

4.1.5.2 InSb Working Standards 13

4.2 Electronics 13

4.2.1 Electronics - Signal Measurement 13

$\begin{array}{ll}\text { 4.2.2 Electronics - Auxiliary Equipment } & 14\end{array}$ 
$\begin{array}{ll}5.1 \text { Alignment } & 15\end{array}$

$\begin{array}{ll}5.2 \text { Sources } & 15\end{array}$

$\begin{array}{ll}\text { 5.2.1 Argon Maxi-arc } & 15\end{array}$

5.2.2 Quartz-Halogen Tungsten Filament Lamp 15

$\begin{array}{ll}5.3 \text { Monochromator } & 15\end{array}$

5.3.1 Relay Optics 15

5.3.2 Spatial Apparatus Function 17

$\begin{array}{ll}\text { 5.3.3 Beam Uniformity } & 17\end{array}$

$\begin{array}{ll}\text { 5.3.4 Wavelength Calibration } & 17\end{array}$

$\begin{array}{ll}\text { 5.3.5 Spectral Characterization } & 19\end{array}$

$\begin{array}{ll}\text { 5.3.6 Temporal Characterization } & 21\end{array}$

$\begin{array}{ll}\text { 5.3.7 Polarization Characterization } & 21\end{array}$

5.3.8 Monitor Detectors 21

5.4 Electronics $\quad 21$

5.4.1 Lock-in Output Gain 21

5.4.2 Lock-in Gain Range Calibration $\quad 22$

5.4.3 Electronic System Linearity Check 22

5.5 Transfer and Working Standards

5.5.1 Bolometer Standard 22

5.5.1.1 Responsivity vs. Time - Cryogenic Effects: cooldown, hold time, and refill 22

5.5.1.2 Responsivity vs. Frequency (including time dependence) 22

5.5.1.3 Responsivity vs. Temperature 24

5.5.1.4 Responsivity vs. Bias Voltage 25

5.5.1.5 Responsivity Nonlinearity 25

5.5.1.6 Dynamic Range 26

$\begin{array}{ll}\text { 5.5.1.7 Spatial Responsivity } & 27\end{array}$

$\begin{array}{ll}\text { 5.5.1.8 Window Properties } & 27\end{array}$

5.5.1.9 Bolometer Gain Range Calibration 28

5.5.1.10 Overall Bolometer Responsivity Correction Equation 28

5.5.2 InSb Working Standards 29

$\begin{array}{ll}\text { 5.5.2.1 Responsivity vs. Time } & 29\end{array}$

$\begin{array}{ll}\text { 5.5.2.2 Responsivity vs. Frequency } & 30\end{array}$

$\begin{array}{ll}\text { 5.5.2.3 Responsivity Nonlinearity } & 30\end{array}$

5.5.2.4 Dynamic Range - Noise Floor 31

5.5.2.5 Spatial/Angular Responsivity $\quad 32$ 
6.1 Bolometer Responsivity Scale 33

6.1.1 Relative Spectral Responsivity 33

6.1.1.1 Bolometer Spectral Absorptance 33

6.1.1.2 Bolometer Window Spectral Transmittance 34

6.1.1.3 Relative Spectral Responsivity 36

6.1.2 Absolute Responsivity Tie Points 37

\begin{tabular}{ll} 
6.1.2.1 IR: Pyroelectric Detector & 37 \\
\hline
\end{tabular}

6.1.2.2 NIR: Ge Detector 39

6.1.2.3 Vis: Si Detectors $\quad 42$

6.1.3 Overall Bolometer Absolute Spectral Calibration and Scale Uncertainty 42

6.2 InSb Working Standard Spectral Responsivity 45

7. OPERATIONAL PROCEDURES AND COMPUTER CONTROL 46

7.1.1 Data Taking Program 46

7.1.2 Bolometer Preparation 46

7.1.3 Detector-under-test Preparation 46

7.1.4 Bolometer - DUT Comparison Procedure (Absolute and Relative Responsivity Measurements)

7.1.5 Spatial Uniformity $\quad 47$

$\begin{array}{ll}\text { 7.1.6 InSb Flashing } & 47\end{array}$

$\begin{array}{ll}\text { 8. FUTURE WORK } & 47\end{array}$

9. ACKNOWLEDGMENTS 47

$\begin{array}{ll}\text { 10. REFERENCES } & 48\end{array}$ 


\section{LIST OF FIGURES}

Figure 3.1 Block diagram of photodetector substitution method.

Figure 4.1 IR SCF overall layout. M indicates offaxis parobolic mirror and MUX indicates a multiplexer. 9

Figure 4.2 The block diagram of the bolometer electronics. 12

Figure 4.3 InSb dewar mount with cold apertures and entrance window. 13

Figure 4.4 The bolometer amplifier shown in the figure is integral with the bolometer dewar. The amplifier for the test detector may or may not have a dedicated amplifier.

Figure 5.1 The effect on shutter blade temperature due to heat from the drive solenoid. The upper curve shows the output signal of the IR SCF when the shutter blades are closed. The lower curve shows the output signal when the shutter is open, and the system sees only the ambient temperature of the room. The monochromator was set to $4.5 \mu \mathrm{m}$.

Figure 5.2 IR SCF spatial apparatus function measured by scanning a $0.25 \mathrm{~mm}$ slit across the monochromator output beam. The signal is shown relative to the detector background level seen as constant across the scan range. 17

Figure 5.3 Prism calibration curve. The position (in encoder-driver steps) of peak prism transmittance versus wavelength. The spectral ranges of the 3 gratings are also indicated.

Figure 5.4 Spectral rejection is shown for a) the $75 \mathrm{~g} / \mathrm{mm}$ grating and b) the $150 \mathrm{~g} / \mathrm{mm}$ grating using two different lasers as sources. The baseline points in b) belong to the $10.6 \mu \mathrm{m}$ curve.

Figure 5.5 Change of $3 \mathrm{~dB}$ roll-off frequency with time. The line indicates the linear fit to the data.

Figure 5.6 Bolometer nonlinearity correction is shown versus output signal level on gain range $\mathrm{x} 20$ for various voltage drops across the bolometer.

Figure 5.7 Bolometer spatial responsivity. A $1 \mathrm{~mm}$ beam is scanned across the bolometer. The points above the double line use the expanded scale on the right to better view the variations of the plateau region.

Figure 5.8 Effect of Bolometer Correction Procedure.

Figure 5.9 Test of InSb stability vs. time at $3.8 \mu \mathrm{m}$.

Figure $5.10 \mathrm{InSb}$ working standard radiometer responsivity vs. frequency normalized to unity at dc.

Figure 5.11 Ratios of InSb to Ge responsivity (for identical radiant power inputs) divided by a constant to yield a mean value near unity.

Figure 5.12 Noise and background current vs. monochromator wavelength.

Figure 5.13 a) InSb horizontal spatial responsivity variation, right side shows expanded scale. b) InSb angular responsivity variation.

Figure 6.1 The solid curve shows the bolometer gold black reflectance measured using a FTIR spectrometer. The reflectance was also measured with laser sources as indicated by the two open squares at $0.633 \mu \mathrm{m}$ and $10.6 \mu \mathrm{m}$.

Figure 6.2 a) Spectral transmittance of a single $\mathrm{KBr}$ window as measured by IR SCF. The mean error bar is $\approx 0.1 \%$ for the region below $16 \mu \mathrm{m}$. b) Spectral transmittance of two $\mathrm{KBr}$ windows and their relative differences. 35

Figure 6.3 The transmittance of a single $\mathrm{KBr}$ window as measured by the Vis/NIR SCF. The lower curve is the standard deviation of the transmittance measurements.

Figure 6.4 Relative spectral responsivity of the bolometer with uncertainties typical of the Vis/NIR and IR spectral regions shown.

Figure 6.5 The radiant power responsivity of the pyroelectric detector used for scale transfer from the HACR to the bolometer.

Figure 6.6 Bolometer spectral radiant power responsivity as transferred using the pyroelectric detector. 39

Figure 6.7 Bolometer responsivity calibration by comparison to a) Si and b) Ge transfer standard detectors. Only the error bar caps are shown for clarity except for the Ge comparison point made using the $1.31 \mu \mathrm{m}$ laser as a source instead of the IR SCF.

Figure 6.8 The radiant power responsivity of the bolometer in the spectral regions tied to the $\mathrm{Si}$ and $\mathrm{Ge}$ transfer standard detectors are indicated by the solid points below $1.8 \mu \mathrm{m}$. For clarity, only the error bars caps are given for these points. The responsivity measurement made using the laser at $1.31 \mu \mathrm{m}$ is indicated by the box and complete error bar. The bolometer responsivity tied to the pyroelectric transfer detector is indicated by the points between $6 \mu \mathrm{m}$ and $11 \mu \mathrm{m}$ with complete error bars. The solid curve is the $\mathrm{T} \cdot(1-\mathrm{R})$ relative responsivity scaled by a factor to give the best overall fit. The two uncertainty error bars for the $T \cdot(1-R)$ curve shown at $1 \mu \mathrm{m}$ and $12 \mu \mathrm{m}$ are due to the uncertainty of the relative spectral data and the spatial responsivity uncertainty of the bolometer. 
Figure 6.9 Ratio of the absolute bolometer radiant power responsivity to the scaled relative responsivity. The error bars indicate the total uncertainty of the ratios, only the error bar caps are shown for clarity. The solid line is the fit to a line.

Figure 6.10 Individual data sets used to determine the scale factor of the relative responsivity curve. The solid points indicate all uncertainty components were assumed statistical. For the open points only the statistical uncertainty components were used in the weights for the fits. The solid and dashed lines indicate the fit to the solid and open points respectively. All data sets are shown in a), while the noisy $1.5 \mu \mathrm{m}$ to $1.7 \mu \mathrm{m}$ data set was removed from $b$ ).

\section{LIST OF TABLES}

Table 5.1 Summary of monochromator spectral widths observed using laser sources. Entries followed by (/2) indicate measurements made using $2 \mathrm{~mm}$ entrance and exit apertures. All other entries were made with $1 \mathrm{~mm}$ apertures.

Table 5.2 Lock-in Amplifier Correction Factors

Table 5.3 Bolometer ac amplifier gain calibration referenced to gain $x 100$

Table 6.1 Bolometer spectral radiant power responsivity uncertainty budget as transferred from the pyroelectric detector. The last column indicates whether the uncertainty is constant over the spectral range or varies with wavelength.

Table 6.2 Bolometer spectral radiant power responsivity uncertainty budget as transferred from the Ge detector. The last column indicates whether the uncertainty is constant over the spectral range or varies with wavelength. 40

Table 6.3 Bolometer spectral radiant power responsivity uncertainty budget as transferred from the Si detector. The last column indicates whether the uncertainty is constant over the spectral range or varies with wavelength. 42 



\section{Introduction}

This document describes a new NIST Measurement Service for providing special tests of infrared (IR) detectors sent to NIST. The main focus of this service is to provide measurements of absolute spectral responsivity (ASR) in the spectral region from $1.8 \mu \mathrm{m}$ to $20 \mu \mathrm{m}$, although other characteristics such as spatial responsivity uniformity can also be measured. This document describes the design philosophy, theory, measurement system, operation, and transfer standards and how the ASR scale is realized and the associated uncertainties.

Many radiometric applications require the determination of the spectral radiant power responsivity function of IR detectors. The spectral power responsivity is the ratio of the detector's output (in amperes or volts) to the spectral radiant flux (in watts) incident on the detector. The spectral power responsivity is also referred to simply as the spectral responsivity. Accurate measurement of spectral power responsivity of photodetectors in the visible spectral region has been a service provided by the Optical Technology Division at NIST for over 20 years. NIST has recently moved to expand the spectral range of the absolute spectral power responsivity measurements provided to its customers, in this case extending the wavelength coverage to $20 \mu \mathrm{m}$.

The High Accuracy Cryogenic Radiometer (HACR) is the U.S. primary standard for detector radiant power calibrations. The scale of spectral power responsivity is transferred by silicon and germanium photodiodes and pyroelectric detectors to an IR cryogenic composite Si bolometer used with the NIST Infrared Spectral Comparator Facility (hereafter referred to as the IR SCF or simply the comparator) where the special test measurement service is performed. This document will briefly review the $\mathrm{HACR}, \mathrm{Si}, \mathrm{Ge}$, and pyroelectric detectors, and the calibration of the bolometer.

The purpose of this document is to introduce the new HACR-based spectral power responsivity scale in the $1.8 \mu \mathrm{m}$ to $20 \mu \mathrm{m}$ spectral range and the measurement methods for testing IR detectors as a calibration service. For this document, the absolute spectral power responsivity scale in the $1.8 \mu \mathrm{m}$ to $20 \mu \mathrm{m}$ spectral range will be referred to as the "IR" detector scale or just the detector scale.

\section{Background}

While NIST has been providing detector responsivity calibrations in the visible spectral region for over two decades, the IR region has been more problematic. In the past 5 years, calibration capability has been extended from the visible out to $1.8 \mu \mathrm{m}$ [PAR96]. Responding to growing interest and developing technology, NIST has moved to extend detector measurement capability further into the IR, out to $20 \mu \mathrm{m}$. While visible and near infrared (NIR) detector calibrations can rely on readily available detectors with excellent radiometric characteristics, the region beyond $1.8 \mu \mathrm{m}$ suffers from the relative lack of such radiometric detectors. Detectors in the IR may have any number of characteristics that can make radiometric calibrations problematic. First, small detector areas can make the required underfilling difficult, while larger areas can increase the background noise and reduce sensitivity. This lower sensitivity is a particular problem in the IR, where the most common thermal sources provide weak outputs, relative to visible sources. 
Spatial responsivity uniformity over a given area can also be quite poor; variations of hundreds of percent have been seen. Spectral responsivity variation is a problem in the IR, in part because that region of the spectrum covers such a wide range. The usual way this is dealt with is by using either a number of higher sensitivity but narrow spectral range detectors or a much lower sensitivity detector with a wide but flat spectral responsivity range [NPW93]. All of these factors have combined to make IR calibrations by national standards laboratories either limited or nonexistent. This situation moved NIST to begin a program to provide coverage of this important range. 


\section{NIST Spectroradiometric Detector Measurement Service}

\subsection{Description of Measurement Services}

The present capabilities for IR photodetector spectral power responsivity measurements cover the range from $1.8 \mu \mathrm{m}$ to $20 \mu \mathrm{m}$. The detector scale relative expanded uncertainty $(k=2)$ is typically $1.6 \%$ throughout this spectral range.[TAK94] The uncertainty of a responsivity calibration of a specific detector will generally be the uncertainties associated with that detector summed in quadrature with the $1.6 \%$ of the detector scale uncertainty.

Note: This document follows, to the extent possible, the "ISO Guide to the Expression of Uncertainty in Measurement" (International Organization for Standardization, Geneva, Switzerland, 1993.) Since 1994, the NIST policy has been to conform to the Guide in reporting its activities, using an expanded uncertainty coverage factor (as defined in the Guide) of $k=2$. See Ref. [TAK94] for a detailed explanation of the NIST policy. In keeping with this policy, we have presented the final results using the expanded uncertainty $(k=2)$, although component uncertainties are given throughout the document are combined standard uncertainties.

\section{Special Tests of IR Detectors (39090S)}

Special tests of radiometric detectors may be performed in the $1.8 \mu \mathrm{m}$ to $20 \mu \mathrm{m}$ spectral region at power levels of about $2 \mu \mathrm{W}$ or less. Examples of detector characteristics that can be determined in a special test include absolute spectral responsivity, relative spectral responsivity, and spatial responsivity uniformity. Since special tests of this type are unique, details of the tests should be discussed with one of the technical contacts before submitting a formal request.

\subsection{Measurement Limitations}

Because of the beam size of the comparator, the detector's active area must be greater than $2 \mathrm{~mm}$ in diameter and have an acceptance $f / \#$ larger than $f / 4\left(\approx 14^{\circ}\right)$. The detector sensitivity and signalto-noise ratio must be compatible with the $\leq 2 \mu \mathrm{W}$ comparator radiant power levels. In general, photodiode shunt resistances must be at least several kohms to meet these sensitivity and signalto-noise requirements. The frequency response $3 \mathrm{~dB}$ rolloff point must be higher than $\approx 50 \mathrm{~Hz}$. To allow for meaningful calibrations, the detector spatial responsivity nonuniformity should be less than $5 \%$.

Physical size and weight are limited by the translation stages used in the IR SCF. Detector packages are limited in size to approximately $50 \mathrm{~cm}$ high by $17.5 \mathrm{~cm}$ in diameter and $15 \mathrm{~kg}$ in weight.

Special tests with conditions greatly differing from those listed above may take several months to schedule. The detector signal (either voltage or current) must be provided via a BNC connector. Computer communication to a customer's electronic package is not possible at this time. 


\subsection{How To Order Special Tests}

1) Reference the Service ID Number on the purchase order.

Details of service ID number 39090 S should be discussed with one of the technical contacts listed in sec. 2.3 prior to submitting a formal request.

2) The purchase order should be sent to:

\section{Calibration Program}

National Institute of Standards and Technology

100 Bureau Dr. STOP 2330

Gaithersburg, MD 20899-2330

Phone number:

FAX Number:

(301) $975-2002$

E-mail:

(301) 869-3548

Internet URL: calibrations@nist.gov

http://s.nist.gov/ts/htdocs/230/233/233.htm

3) The purchase order must include the following:
A) Bill to address.
B) Ship to address (for the test detector(s) return delivery).
C) Method of return shipment (the costs below do not include shipping costs.) If nothing is stated, NIST will return by common carrier, collect, and uninsured.
D) User's name, address (for test report), and phone number.
E) Service ID number 39090S.

4) The cost for special tests is based on the actual labor and material costs involved and customers are responsible for all shipping costs.

\subsection{Technical Contacts}

All test detector(s) should be shipped to the following address:

\section{George Eppeldauer \\ National Institute of Standards and Technology \\ 100 Bureau Dr. STOP 8441 \\ Gaithersburg, MD 20899-8441}

For technical information or questions contact:

$\begin{array}{ll}\text { George Eppeldauer } & (301) 975-2338 \\ \text { Thomas Larason } & (301) 975-2334 \\ \text { or fax } & (301) 869-5700\end{array}$

email: george.eppeldauer@nist.gov email: thomas.larason@nist.gov 
Technical information can also be found at the following Internet URL:

http://physics.nist.gov/Divisions/Div844/facilities/phdet/phdet.html

\section{Measurement Theory}

The general measurement method, the measurement equation, the realization of the NIST detector spectral responsivity scale, and the transfer of this scale to customer detectors are only briefly discussed here. For more complete details of this theory, see SP 250-41 by Larason et al. [LBP98]. The Infrared Spectral Comparator Facility (IR SCF) is entirely based on the substitution method to transfer radiant power responsivity of a standard to an unknown detector [CIE84, BUD83].

\subsection{Substitution Method}

The substitution method transfers the responsivity of a standard detector (output divided by input) to an unknown (test) detector. This method has a number of advantages and some limitations in the transfer of a detector responsivity scale. These characteristics will be discussed specifically for the IR SCF.

\subsubsection{General Substitution Method}

The output (signal) $Y$ from a detector (or system) with a linear responsivity in $\mathrm{U}$ units, typically volts or amperes, can be written in general,

$$
Y_{x}=S_{x} \cdot X_{x}[\mathrm{U}]
$$

where $Y_{\mathrm{x}}$ is the signal from the detector, $S_{\mathrm{x}}$ is the responsivity of the detector, and $X_{\mathrm{x}}$ is the input to the detector. The signal from a standard detector with a known responsivity $S_{\mathrm{s}}$ is given by,

$$
Y_{s}=S_{s} \cdot X_{s}[\mathrm{U}]
$$

Dividing eq (1) by eq (2) we have,

$$
\frac{Y_{x}}{Y_{s}}=\frac{S_{x} \cdot X_{x}}{S_{s} \cdot X_{s}}
$$

If the input $X$ to each system is assumed to be the constant (over the time of the two measurements), solving for the detector responsivity $S_{\mathrm{x}}$, gives:

$$
S_{x}=\frac{Y_{x}}{Y_{s}} \cdot S_{s}\left[\mathrm{U} \cdot \text { input units }{ }^{-1}\right] .
$$

Thus, with the substitution method the standard detector responsivity $S_{\mathrm{s}}$ is scaled by the ratio of the outputs. The transfer is accomplished in essence by the standard detector measuring the input $X$ to the test detector. 


\subsubsection{Detector Substitution}

Detector responsivity measurements by substitution can be made using eq (4) and the following equipment: a broadband source, monochromator, focusing optics, and standard detector. (See fig. 3.1.) The radiant flux (power) $\Phi$ is the output flux from the source that enters the monochromator. The spectral radiant flux $\Phi_{\lambda}$ is the output flux from the monochromator and $\tau$ is the transmission of any optics between the monochromator and the detector. The spectral radiant flux received by the detectors is $\Phi_{\mathrm{D}, \lambda}=\tau \cdot \Phi_{\lambda}$.

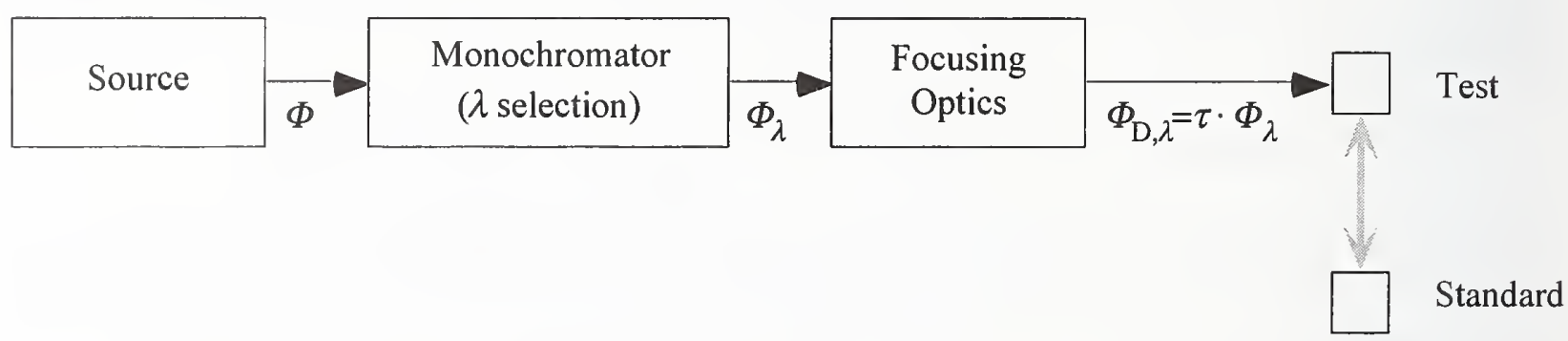

Figure 3.1 Block diagram of photodetector substitution method.

But using eq (4) assumes that the source is stable over the comparison time. This is typically true to better than a few percent for the sources used by the IR SCF. Source fluctuations during the comparison should be statistical allowing their effect to be reduced by averaging repeated measurements.

\subsection{Measurement Equation}

Developing the measurement equation is fundamental to understanding the measurement process and the assumptions or approximations that are made. The measurement equation is also fundamental in the analysis of the uncertainty of the measurement process. In essence a detector comparator is similar to a spectroradiometer, except that it measures the spectral responsivity of different detectors instead of the spectral power distribution of different sources. Thus, the measurement equation developed for a general spectroradiometer can be adapted to our situation of detector spectral responsivity measurements.

Equation (4) for the test detector spectral responsivity $S_{\mathrm{x}}$ can be made more explicit by including the zero subtraction $\left(V_{X}^{0}, V_{S}^{0}\right)$ and detector gain terms $\left(G_{X}, G_{S}\right)$ for the test and standard detectors as follows

$$
S_{X}=\frac{V_{X}-V_{X}^{0}}{V_{S}-V_{S}^{0}} \cdot \frac{G_{S}}{G_{X}} \cdot S_{S}\left[\mathrm{U} \cdot \mathrm{W}^{-1}\right] .
$$

This is the working form of our measurement equation. 


\subsubsection{Approximations}

The assumptions made in the development of the measurement equation are summarized as follows. The development of the measurement equation, which followed the procedure described in Chapter 5 of the Self-Study Manual on Optical Radiation Measurements [KON78], does not include parameters for time, polarization, angle, environmental parameters (such as ambient temperature or humidity), corrections for diffraction effects (departure from geometrical ray optics), and nonlinear responsivity. These assumptions are discussed below, and the resulting uncertainties are analyzed in Section 5.

A key requirement for detector-based radiometry is that detector responsivities are stable over time. In general the light from any monochromator system can be polarized, thus the effects of polarization on the responsivity of the detectors needs to be evaluated. Generally this is not a problem, because most detectors are measured at normal incidence to the optical axis and detector surfaces are usually isotropic. For detectors with polarization dependent responsivity, the polarization of the IR SCF output beam must be taken into account. The effect of the converging beam angle on the reflectance (and transmittance) of the detector surface (and window) is small compared to the variance of repeated measurements and is typically neglected. This is not the case when filters are used with the detectors (e.g., photometers), especially interference filters whose transmittance is a strong function of the angle of incidence. Also, the detector area must be larger than the optical beam so that all of the optical radiation is collected by the detector (i.e., the detectors are underfilled). This also requires that the detector Field-ofView (FOV) be larger than the optical beam from the comparator system.

Humidity affects the measurement by changing the transmittance $\tau$ of the system. This is usually minimized by purging the system with dry gas. Humidity may also effect the detector itself (or its window). The typical windowed detector is not effected by water absorption (into the detector or window). Both the humidity and temperature variations are small over the measurement time; and, therefore, their effects on the responsivity are neglected over most of the spectral region. When it is a concern, it can be treated as an additional uncertainty term.

Diffraction effects can be estimated from [HEC87],

$$
\theta^{d}=2.44 \cdot \frac{\lambda}{d}[\mathrm{rad}],
$$

where $\theta^{d}$ is the diffraction angle (for the first Airy disk), $\lambda$ is the longest wavelength of interest, and $d$ is the diameter of the aperture at the monochromator exit slit (the smallest aperture in the system). For the IR SCF, with $d \approx 1 \mathrm{~mm}$ we get, $\theta^{d}=5 \mathrm{mr}$ and $50 \mathrm{mr}$ respectively at the $1.8 \mu \mathrm{m}$ and $20 \mu \mathrm{m}$ ends of the IR SCF spectral range. These are 50 to 5 times larger than the diffraction limit, so at the shorter wavelength end of the range the effect can be neglected and at the longest wavelength end a slight correction may be required. Likewise coherence effects are negligible [SHU83] and are ignored in the analysis of this comparator system.

The responsivity was assumed uniform throughout the active area of the detector. In practice this can be approximated by reducing the area (both mechanically with apertures and optically 
via imaging optics). This limits the amount of flux (power) that can be delivered to the detector and can limit some of the measurement possibilities. Deviations from this approximation can be considered as an uncertainty term.

Another assumption about the IR SCF is that the dispersion of the monochromator is the same for all wavelengths and that the slit-scattering function $z\left(\lambda_{0^{-}} \lambda\right)$ does not change with the monochromator wavelength setting $\lambda_{0}$ [KOS79]. This allows $z\left(\lambda_{0}-\lambda\right)$ to be measured only once for an instrument and used over the entire wavelength range. (This assumption is tested in sec. 5.3.5.) Related to this assumption is that optical aberrations, scattering, and diffraction are the same whether the $\lambda$ of a monochromatic source is varied over the monochromator bandpass $\delta \lambda$ or whether the monochromator setting $\lambda_{0}$ is scanned over a monochromatic source of wavelength $\lambda$. Also $\mathrm{z}\left(\lambda_{0}-\lambda\right)$ was assumed approximately symmetrical with respect to $\lambda_{0}$ which is the case for the monochromators described in this publication.

Detector linearity is assumed in the measurement equation eq (5). In practice, detector nonlinearity is tested and kept at small levels to either eliminate any effect or allow for very small corrections with correspondingly small correction uncertainties.

The spectrally scattered light is generally considered small. This is checked by testing the effect of the addition of cutoff filters on the final determined detector responsivity. The size of this variation indicates the level of spectral stray light in the system.

\section{Equipment Description}

\subsection{Infrared Comparator Description}

\subsubsection{Comparator Facility Description Overview}

The IR spectral comparator facility is a monochromator based system that can measure the absolute spectral responsivity of detectors in the wavelength region from $1.8 \mu \mathrm{m}$ to $20 \mu \mathrm{m}$. The facility essentially consists of three components, an IR source, the monochromator and a transfer standard detector. A cryogenic silicon-sapphire composite bolometer serves as the transfer standard for the IR SCF. The spectral comparator uses the direct substitution method. The detector under test and the working standard are exchanged in the monochromator output beam using automated translation stages to position the detectors for measurement. The test detectors as well as the working standard are fixed or kinematically mounted to sturdy optical mounts. The detector positions are reproduced accurately during the measurement sequence. Most often an argon arc is used as the light source for the IR SCF, although a $100 \mathrm{~W}$ quartz-halogen lamp can be used if required. The sources, spectrometer, detectors, and the optical paths can be purged with dry gas if needed. A diagram of the IR SCF is shown in Figure 4.1. 


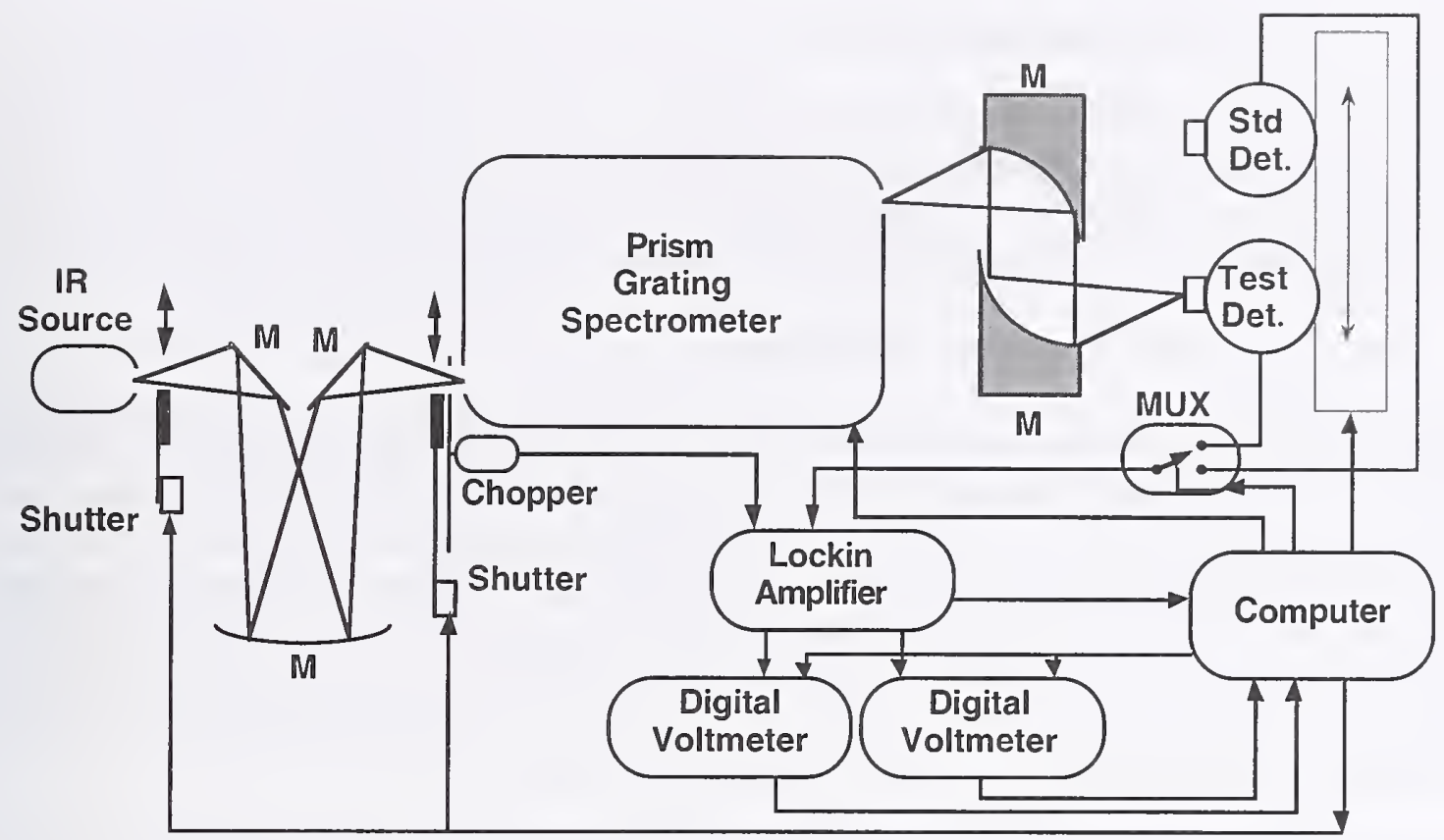

Figure 4.1 IR SCF overall layout. $M$ indicates a mirror and MUX indicates a multiplexer.

\subsubsection{Description of Sources}

\subsubsection{Ar Maxi-arc}

The IR SCF generally uses a NIST designed argon maxi-arc as its source. The argon maxi-arc was originally developed as a secondary spectral radiance standard in the UV and it has been well characterized for that spectral region [BRO77, KBO88]. It was adapted for use in the IR SCF because it is a high temperature and good stability source. A detailed discussion of this source can be found elsewhere and is just briefly described here [BRM95]. The discharge is run between two electrodes in a $4.7 \mathrm{~mm}$ diameter by $50 \mathrm{~mm}$ long channel purged with Ar gas. The channel is made from seven stacked insulated plates bored with a single hole in the center of each. Gas ports allow Ar gas to be introduced into the center of the discharge and exhausted at ambient pressure out both ends of the channel and through side ports in some of the channel defining plates. The discharge was current stabilized at $90.00 \mathrm{~A} \pm 0.01 \mathrm{~A}$ with the dc voltage $\approx 90 \mathrm{~V}$. Chilled water at $6 \mathrm{C}$ removes most of the $8 \mathrm{~kW}$ of heat generated by the discharge. The discharge region, which is viewed end on, radiates into a cone somewhat greater than $f / 6$, limited by the channel and discharge region aspect ratios. The arc source produces light from the UV to beyond $20 \mu \mathrm{m}$. Below about $4 \mu \mathrm{m}$ some line structure is seen in the arc spectrum. Beyond $4 \mu \mathrm{m}$ the spectrum becomes smoother and approaches the spectrum of a $10000 \mathrm{~K}$ blackbody. 


\subsubsection{Quartz Lamp}

A Gilway* part number L7390, $100 \mathrm{~W}$ current controlled quartz-halogen lamp can also be used as an IR source for the monochromator. This lamp is typically used for the near infrared end of the spectral range where maximum spectral power is not always required. In addition, the spectrum of this source is much smoother than the maxi-arc in the near infrared region.

\subsubsection{Safety considerations}

Quartz-halogen lamps, lasers, and the argon arc are each potential eye hazards. UV filtering safety glasses must be worn whenever the argon arc source output is visible. Even with safety glasses on, argon arc is never viewed directly. Precautions are taken when striking the arc, such as not shorting the input to ground and not coming in direct contact with the leads by making sure that insulation exists between the operator and the bare contacts. Also, attention is paid to the water lines, making sure the water is flowing whenever the arc is on and that no water leaks exist. Only nonconducting plumbing is connected to the arc. An exhaust hood is used to remove ozone produced by the arc.

Shields and laser curtains mounted on the sides of the optical table containing the entire IR SCF optical system inhibit direct eye contact with the sources. The lasers used for alignment are Laser Safety Class II. In addition, lasers at $3.39 \mu \mathrm{m}$ (Class IIIb) and $10.6 \mu \mathrm{m}$ (Class IV) are used for wavelength calibration of the monochromator. Protective eyeware is worn when working with sources that are potential eye hazards.

\subsubsection{Monochromator}

Spectral selection for the IR SCF is achieved with an IR prism-grating monochromator. The prism portion of the monochromator provides bandwidth limiting and order sorting for the grating portion. The wavelength is varied by rotating the grating and prism simultaneously under computer control. A single KRS-5 prism is used to cover the entire IR spectral region, while two gratings, $150 \mathrm{~g} / \mathrm{mm}$ and $75 \mathrm{~g} / \mathrm{mm}$, (with dispersions of $\approx 30 \mathrm{~nm} / \mathrm{mm} \approx 60 \mathrm{~nm} / \mathrm{mm}$ respectively) are used to cover the IR spectral region. The crossover point in throughput efficiency between these two gratings is about $7 \mu \mathrm{m}$. A set of filter wheel mounted long wavepass filters can be placed at the output of the monochromator to test and if necessary improve second order rejection. These filters have cut-on wavelengths of $2.0 \mu \mathrm{m}, 3.75 \mu \mathrm{m}, 6.5 \mu \mathrm{m}$, and $11.7 \mu \mathrm{m}$ and blocking of $0.1 \%$. In addition to the two IR gratings, a $600 \mathrm{~g} / \mathrm{mm}$ grating is also available to allow extension of the tunable region into the near IR and visible.

The design specifications for the monochromator grating are as follows: focal length is $198 \mathrm{~mm}$, spectral resolution is $1 \%$, the acceptance angle is $f / 4$, and the wavelength accuracy is $\pm 5 \mathrm{~nm}$.

\footnotetext{
* Certain trade names and company products are mentioned in the text or identified in an illustration in order to adequately specify the experimental procedure and equipment used. In no case does such identification imply recommendation or endorsement by the National Institute of Standards and Technology, nor does it imply that the products are necessarily the best available for the purpose.
} 
The monochromator has entrance and exit apertures from $0.4 \mathrm{~mm}$ to $2.0 \mathrm{~mm}$ to set the beam spot size. The beam is imaged at $f / 4$ onto the detector.

\subsubsection{Optical Relay System and Associated Hardware}

A schematic diagram of the optics for the IR SCF is shown in Figure 4.1. The optical source is imaged 1 to 1 onto the entrance aperture using a $23 \mathrm{~cm}$ focal length spherical mirror. Two flat mirrors are used to steer the object and image beams. A chopper is located within $1 \mathrm{~cm}$ of the entrance aperture. Two Uniblitz shutters are operated in tandem to minimize heating of the back side of the shutter seen by the entrance aperture. In the typical configuration for measurements, the monochromator has a circular $1.0 \mathrm{~mm}$ aperture, although other apertures can be substituted. The exit of the monochromator is imaged 1 to 1 onto a detector using a pair of bare aluminum, diamond-turned, off-axis paraboloids with an effective focal length of $77 \mathrm{~mm}$. The $f$ /\# of the beam incident on the detectors is set by an iris (not shown in Figure 4.1) mounted on the paraboloid mirror assembly.

\subsubsection{Translation Stages - Detector Positioning}

The transfer or working standard and the test detector are positioned horizontally and vertically using computer controlled stages. The maximum travel of the vertical stage is $15 \mathrm{~cm}$ with an accuracy of $4 \mu \mathrm{m}$ per $25 \mathrm{~mm}$ and an overall repeatability of $4 \mu \mathrm{m}$. The maximum travel of the horizontal stage is $40 \mathrm{~cm}$ with a step size of $2.5 \mu \mathrm{m}$, an accuracy of $2 \mu \mathrm{m}$ per $25 \mathrm{~mm}$ and an ovrall repeatability of $2 \mu \mathrm{m}$.

\subsubsection{Detector Alignment}

For initial detector alignment a tiny incandescent bulb is placed within the monochromator just behind to the exit aperture. The visible image of the exit slit can then be visually superimposed on the detector active area. After this alignment the bulb is removed from the optical path. The final alignment is performed while observing the detector output signal.

\subsubsection{Alignment Lasers}

A $5 \mathrm{~mW} \mathrm{HeNe} \mathrm{laser} \mathrm{is} \mathrm{used} \mathrm{to} \mathrm{align} \mathrm{the} \mathrm{optical} \mathrm{path} \mathrm{of} \mathrm{the} \mathrm{comparator.} \mathrm{The} \mathrm{laser} \mathrm{is} \mathrm{arranged} \mathrm{to}$ illuminate the entrance aperture and then to hit the first mirror in the spectrometer on center. This defines the input optical axis. $\mathrm{A} \mathrm{CO}_{2}$ laser at $10.6 \mu \mathrm{m}$ and an IR HeNe at $3.39 \mu \mathrm{m}$ are also available for alignment use with the IR SCF.

\subsubsection{Enclosure}

The spectrometer has a light darkening box designed to reduce the background signal caused by stray room light. This box is continually purged with dry $\mathrm{CO}_{2}$ free air to reduce atmospheric IR absorption. In addition, an enclosure consisting of black curtains surrounds the entire optical table on which the IR SCF is mounted, forming a four sided box with a top. This curtained enclosure houses the test artifacts, the transfer and working standards, the relay optics, and the translation stages. 


\subsubsection{Transfer and Working Standards}

\subsubsection{Cryogenic Bolometer Standard}

The primary transfer standard for the IR SCF is a composite cryogenic bolometer operated near $4 \mathrm{~K}$. The bolometer consists of a sapphire disk with an absorptive coating on one side and temperature sensor on the other side. Incident power absorbed by the coating is detected as a rise in temperature. The absorber is gold black optimized to have low spectral reflectance throughout the IR region. The temperature sensor is a doped silicon chip with a temperature dependent resistance. The $5 \mathrm{~mm}$ diameter bolometer disk is located behind a $3.5 \mathrm{~mm}$ cold aperture and baffling to provide an $f / 4$ acceptance angle. The aperture and baffling are cooled to near $4 \mathrm{~K}$ [EMC93]. The window for the bolometer dewar is made of $\mathrm{KBr}$ and has a wedge angle of $3^{\circ}$. This angle was chosen so as to prevent the primary and secondary transmitted beams from simultaneously falling within the $3.5 \mathrm{~mm}$ bolometer aperture.

The IR SCF control system and bolometer circuit are described here briefly as they are described elsewhere in detail [EMG95](see Figure 4.4). Incident radiation is detected as a change in bolometer resistance. The resistance is monitored by biasing the resistor with a dc current of 1.4 $\mu \mathrm{A}$. Two outputs are recorded, the amplified ac signal indicative of the incident chopped IR radiation and the dc voltage which depends on the quiescent thermal load. The bolometer's dc voltage drop is used to correct for responsivity changes caused by changes in total thermal loading or changes in the liquid He boiling point. To avoid adding noise to the circuit, the bolometer's dc voltage drop is not measured directly. Instead, $\mathrm{a} \approx 70 \mathrm{~K}$ unity gain junction field effect transistor (JFET) impedance transformer provides isolation. The dc voltage drop across the bolometer is found by measuring the change in voltage of one lead of the bolometer (seen via the JFET) as the bias current is turned on and off under computer control. (By measuring this difference, the constant voltage drop of the JFET drops out.) The ac signal from the bolometer is amplified by a room temperature circuit integral with the bolometer dewar with nominal voltage gain values of 20,100,200 and 1000. In addition to these outputs, the bolometer bias voltage is also recorded.

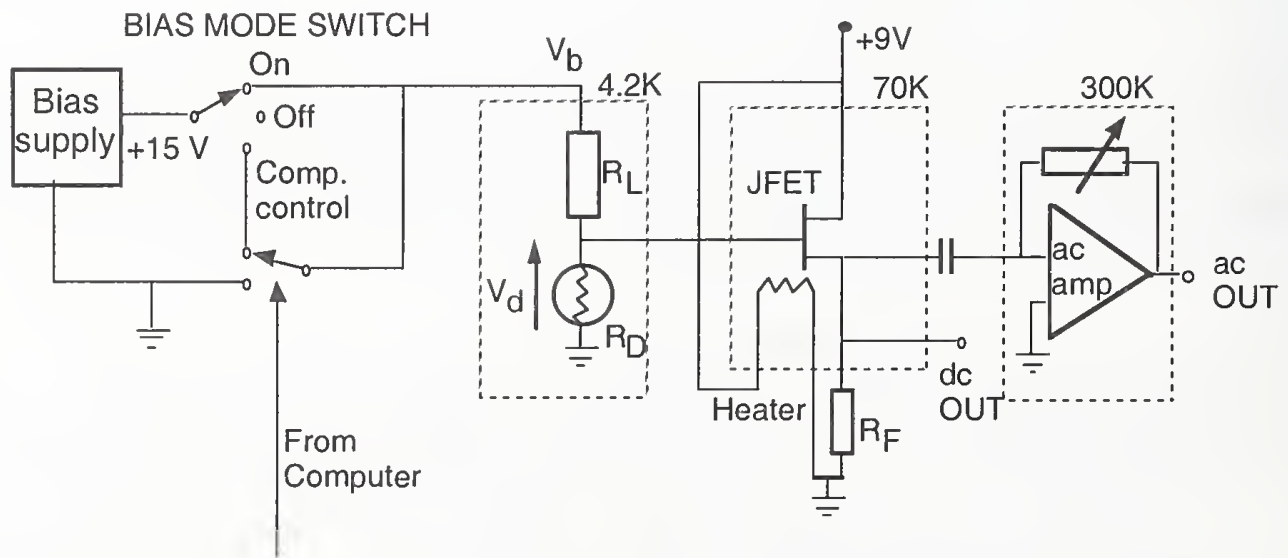

Figure 4.2 The block diagram of the bolometer electronics. 


\subsubsection{InSb Working Standards}

Photovoltaic InSb detectors are used as working standards for the $2 \mu \mathrm{m}$ to $5.4 \mu \mathrm{m}$ wavelength region. The diameter of the active area is $4 \mathrm{~mm}$ with a $3.5 \mathrm{~mm}$ precision aperture mounted within $1 \mathrm{~mm}$ of the detector surface. The detectors are cooled in liquid $\mathrm{N}_{2}$ dewars which have holders for optional cold filters. The shunt resistance at $77 \mathrm{~K}$ is $1 \mathrm{M} \Omega$ and the junction capacitance is $6 \mathrm{nF}$. The front geometry of the InSb detector-dewar is shown in Figure 4.3. A second aperture is located in front of the precision aperture at a distance of $\approx 50 \mathrm{~mm}$. The two cold apertures limit the field of view to $17^{\circ}$, yielding an overall viewing angle of $\approx 26^{\circ}$ as shown in the figure. The precision aperture can be either underfilled or overfilled by the $f / 4$ output beam of the monochromator. Radiant power responsivity calibrations require the precision aperture to be underfilled. A $3^{\circ}$ horizontally wedged window is located at the front of the dewar snout allowing the detectors to be used for the measurement of both coherent and incoherent radiation. The current measuring preamplifier is attached to the outside of the dewar. The low noise preamp is integral with the detector dewar. It has manually selectable gains of $10^{4}$ to $10^{7} \mathrm{~V} / \mathrm{A}$ with a $3 \mathrm{~dB}$ point greater than $400 \mathrm{~Hz}$.

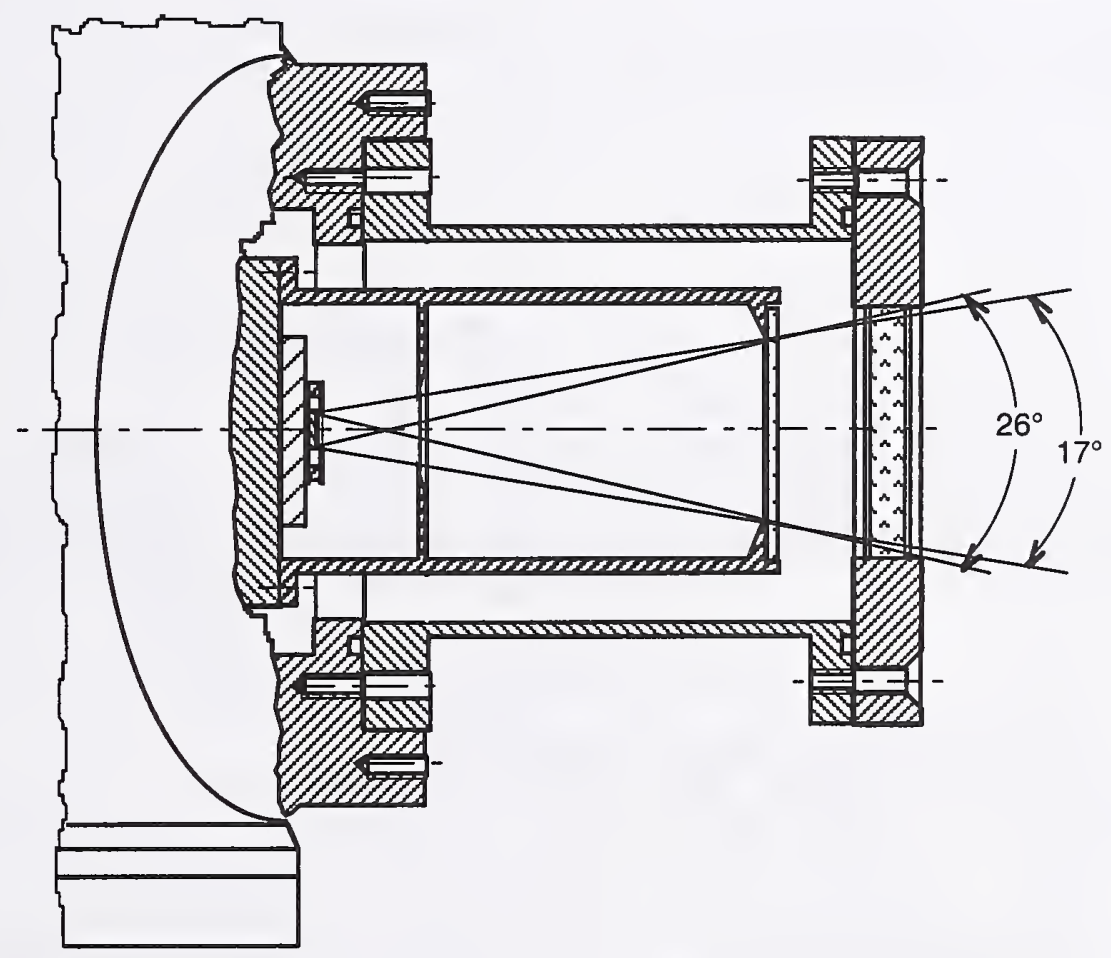

Figure 4.3 InSb dewar mount with cold apertures and entrance window.

\subsection{Electronics}

\subsubsection{Electronics - Signal Measurement}

As mentioned in sec. 4.1.5.1., computer controlled relays turn the bolometer bias current on and off. (An overview of the measurement data flow and control is shown in Figure 4.4.) The bolometer dc output voltage is recorded for the bias on and off states via a multiplexing DMM. 
Due to long dc time constants, best results are obtained by waiting $50 \mathrm{~s}$ between bias current switching and recording of the dc voltage. This dc measurement is not performed for every optical measurement because of the long time required for the dc measurement sequence. Typically it is performed only once every 15 ac optical measurements.

The ac bolometer output is sent via a multiplexer to a dual phase sine wave lock-in amplifier. (This is a PC card lock-in mounted in a 386 computer and controlled through the serial port.) The lock-in amplifier has five gain ranges from $0.1 \mathrm{mV}$ to $1 \mathrm{~V}$ full scale in steps of 10 . In addition, the lock-in output has a 10x nominal gain. The inphase and quadrature analog lock-in outputs (labeled $\mathrm{i}$ and $\mathrm{q}$ in Figure 4.4) are recorded by two simultaneously triggered DMMs which serve as $\mathrm{A} / \mathrm{D}$ converters for the lock-in. These two channels are summed in quadrature digitally by the computer to extract the signal magnitude. Low pass filtering of the analog signals is achieved by the integrating time constants of both the lock-in outputs and the DMMs averaging times. In general the lock-in output time constant is set to $333 \mathrm{~ms}$, although it may range from $3 \mathrm{~ms}$ to $3.3 \mathrm{~s}$. The DMMs are always operated with an averaging time of 100 powerline cycles (i.e., $100 / 60 \mathrm{~s})$

The signal from a detector under test is sent to an appropriate amplifier followed by the same multiplexer and lock-in as that of the bolometer.

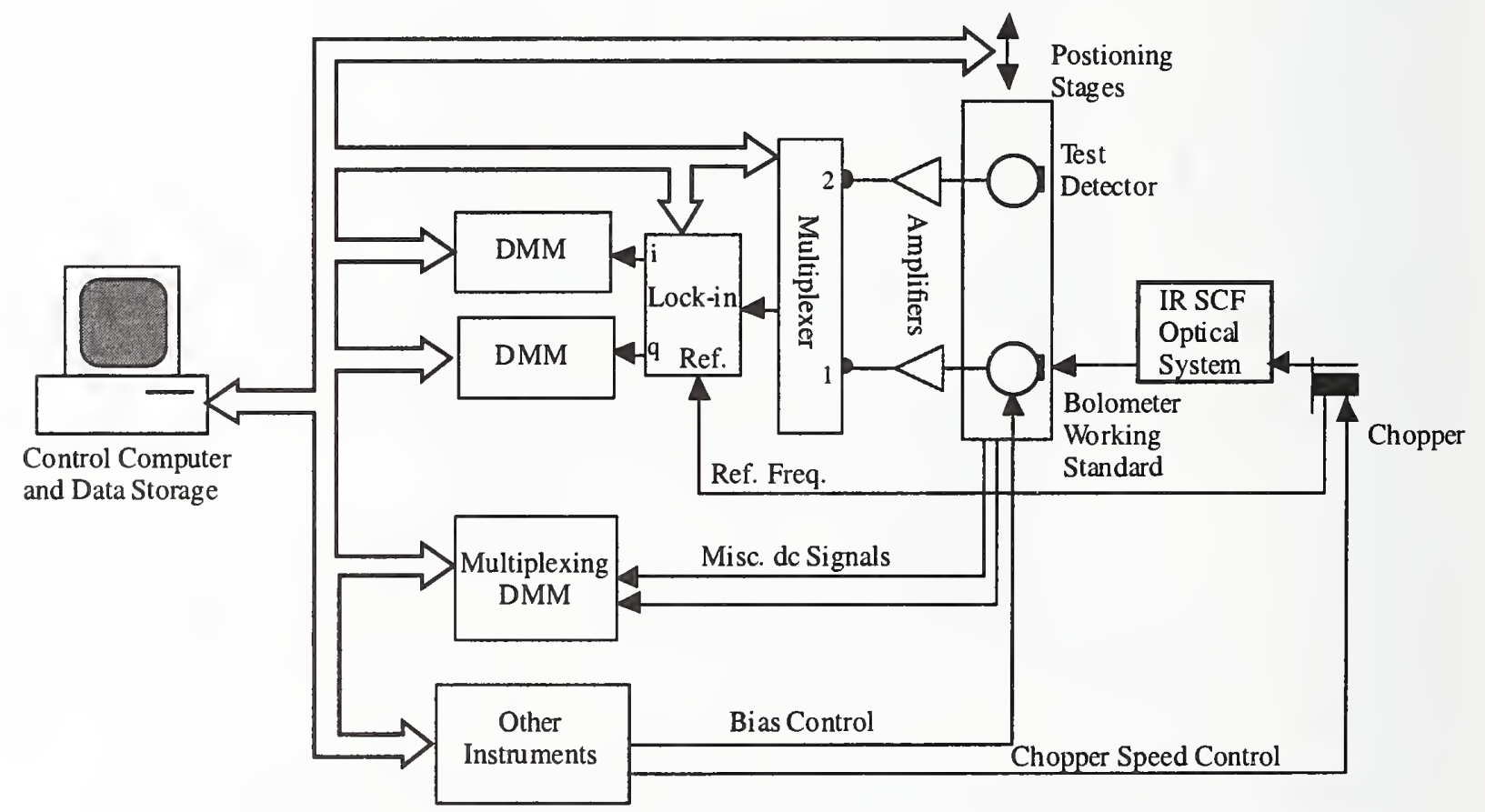

Figure 4.4 The bolometer amplifier shown in the figure is integral with the bolometer dewar. The amplifier for the test detector may or may not have a dedicated amplifier.

\subsubsection{Electronics - Auxiliary Equipment}

Other electronics used with the IR SCF include the chopper and shutter controllers. The chopper speed may be controlled by the computer through an analog voltage. Alternatively, if the voltage 
input line is not connected, the speed can be controlled manually by a knob on the front panel. The two shutters are operated in parallel by a single driver which is controlled by a digital I/O line from the computer.

\section{Equipment Characterizations}

\subsection{Alignment}

The source is initially aligned along the input optical axis as defined by the input alignment laser described in sec. 4.1.4.3. The source is moved along the optical axis to minimize the spot size at the input aperture. Final focus, horizontal, vertical and tilt adjustments are made by simply maximizing the signal at the exit aperture of the spectrometer as seen by a detector.

As mentioned in sec. 4.1.4.2. the detectors are initially aligned using the small incandescent bulb to illuminate the exit aperture. The detectors are positioned visually with the smallest spot centered on the detector. The detector tilt is important, especially for the bolometer which has an $f / 4$ baffle arrangement. To orient the bolometer correctly, the visible beam spot is centered on the wedged window bolometer while simultaneously centering the spot on the $3.5 \mathrm{~mm}$ bolometer aperture. To verify the alignment, the bolometer position is scanned horizontally and vertically. Proper orientation is indicated by a flat region typically over a region of $\approx 1 \mathrm{~mm}$ where the signal is constant to $\approx 1 \%$.

\subsection{Sources}

\subsubsection{Argon Maxi-arc}

The Ar maxi-arc has been characterized for spectral output and stability. The arc output was found to vary on the order $1 \% / \mathrm{h}$ over long periods with $\approx 1 \%$ noise seen over times of a few minutes. The output dependence versus ambient pressure and discharge current were measured at several wavelengths. For complete details, see Ref. BRM95.

\subsubsection{Quartz-Halogen Tungsten Filament Lamp}

Tungsten lamps similar to the one used by the IR SCF have been characterized elsewhere. Typical drifts for these type of lamps are $0.1 \%$ in $10 \mathrm{~h}$. For details, see Ref. OHN97.

\subsection{Monochromator}

\subsubsection{Relay Optics}

The off-axis paraboloids used to image the output aperture of the spectrometer onto the detector were characterized for scatter. The scatter measurement was made in the visible, providing an upper limit on the IR scatter, since it is expected that visible scatter would exceed any IR scatter. A diffusive scatter on the order of about $0.5 \%$ was found. This measurement was made with the pair of mirrors operating in the configuration in which they are actually used for detector comparisons. 
The input optics consisting of one spherical and two fold mirrors are just used to image as much of the source radiation into the input aperture as possible. Scatter effects at this point were not measured because this is unchopped radiation and thus would not affect the accuracy of the detector comparison.

The chopper was positioned as close to the input aperture as possible $(\approx 1 \mathrm{~cm})$. This reduced the diameter of the beam at the chopper relative to the chopper blade opening, which reduces rounding of the squarewave shape of the optical signal. This position also minimizes the amount of chopped background radiation. It is important that no chopped radiation reaches the detectors without passing through the monochromator as otherwise this would induce a spectral measurement error.

The two shutters, which operate in tandem, were placed along the optical path, with one shutter near the source and one near the spectrometer input aperture. This arrangement minimized the heat load on the shutter seen by the spectrometer, reducing the zero level. In addition, the shutter viewed by the monochromator can have slightly different equilibrium temperatures due to its operating mode and solenoid heating. When the shutter is open its solenoid is activated, warming the blades. There is no heating when the shutter is closed and the blade temperature approaches ambient. It is necessary to maintain the temperature balance of the blade during a measurement. A test was made to measure the signal and the zero levels using only the ambient room as a source. Figure 5.1 shows the change of the zero and the signal levels as the shutter goes from the equilibrium closed-mode temperature to the periodic opened-closed mode temperature. It took about an hour to get the temperature balance of the blade, which shifted the result by $\approx 7 \mathrm{pW}$, whereas the signal due to the room background was constant to within $0.5 \mathrm{pW}$. This effect should be considered when the signal is smaller than $1 \mathrm{nW}$.

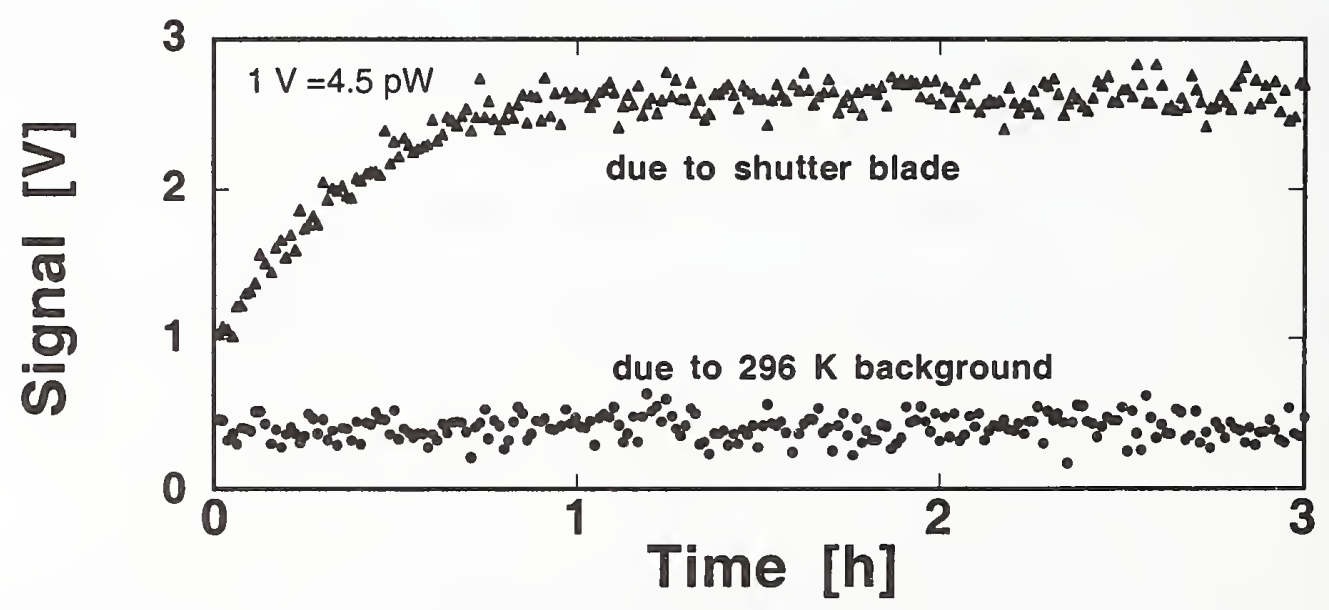

Figure 5.1 The effect on shutter blade temperature due to heat from the drive solenoid. The upper curve shows the output signal of the IR SCF when the shutter blades are closed. The lower curve shows the output signal when the shutter is open, and the system sees only the ambient temperature of the room. The monochromator was set to $4.5 \mu \mathrm{m}$. 


\subsubsection{Spatial Apparatus Function}

The spatial apparatus function was measured by scanning a Ge detector, covered by an aperture with a $200 \mu \mathrm{m}$ pinhole, across the nominal $1 \mathrm{~mm}$ image of the spectrometer output aperture. This measurement was done in the VIS/NIR region using the spectrometer with its $600 \mathrm{~g} / \mathrm{mm}$ grating. The results showed a $0.8 \mathrm{~mm}$ FWHM and a $1.3 \mathrm{~mm}$ full width at $1 \%$ of the maximum. The measurements covered over 3 decades of dynamic range.

The spatial apparatus function was also measured at $10.6 \mu \mathrm{m}$ by scanning a $\mathrm{HgCdTe}$ detector and a $0.25 \mathrm{~mm}$ slit across the image of the spectrometer output aperture. For this measurement a $2 \mathrm{~mm}$ exit aperture was used. A $\mathrm{ZnSe}$ lens was used to image the aperture onto the detector slit with a magnification of 1.18 , so a $2.36 \mathrm{~mm}$ image was expected. The results are shown in Figure 5.2 with the background level shown for comparison purposes. The measured full widths at the half, $10 \%, 1 \%$, and $0.1 \%$ maximums were $1.2 \mathrm{~mm}, 1.6 \mathrm{~mm}, 1.9 \mathrm{~mm}$, and $2.3 \mathrm{~mm}$, respectively.

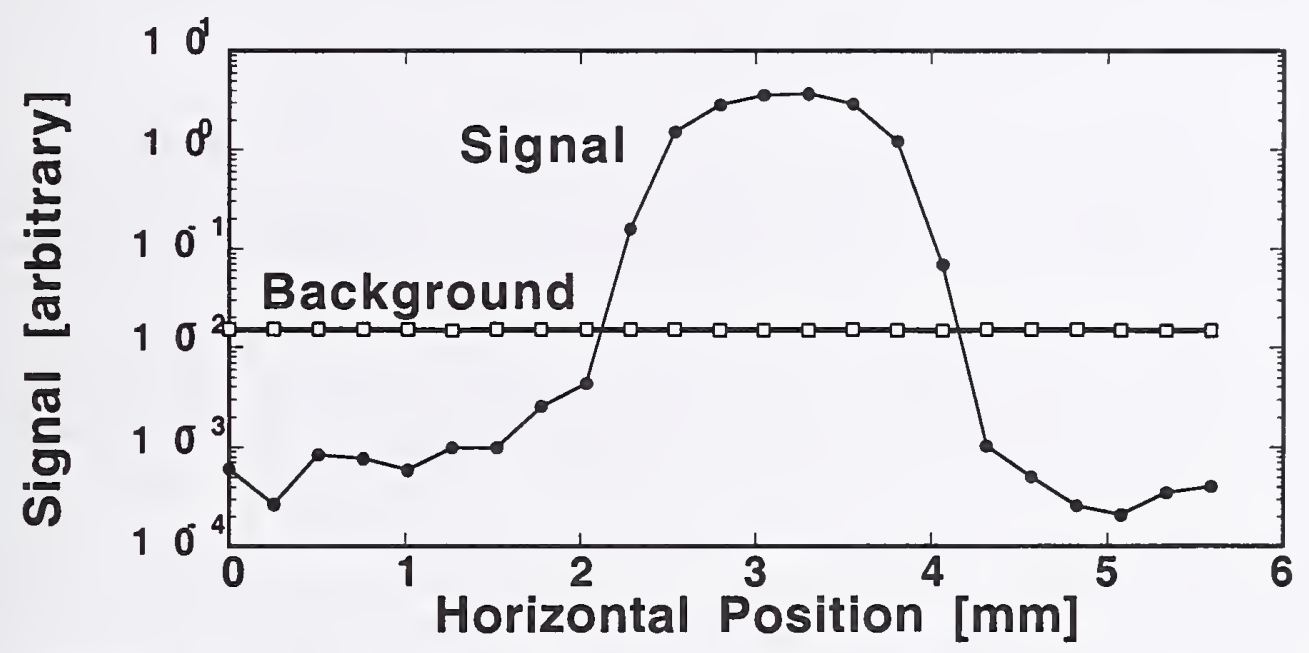

Figure 5.2 IR SCF spatial apparatus function measured by scanning a $0.25 \mathrm{~mm}$ slit across the monochromator output beam. The signal is shown relative to the detector background level seen as constant across the scan range.

\subsubsection{Beam Uniformity}

This facility is intended for operation with the test detector underfilled by the output beam of the spectrometer. In this configuration the beam uniformity is not specified, although it is expected to be a smooth function. Even if this were not the case, it is not likely to have a significant effect on the final spectral radiant power measurement results. Scans of detector uniformity using the IR SCF can be used to provide some information on the combination of beam and detector nonuniformity.

\subsubsection{Wavelength Calibration}

The first step in setting the wavelength calibration of the spectrometer uses the grating equation. This provides an estimate of the wavelength versus angle function for the gratings in our spectrometer. An empirical correction is made to this function by measuring the positions of two 
known wavelength laser lines. The small measured shifts are fit to a linear function which is added to the grating function. Generally the $3.39 \mu \mathrm{m}$ and $10.6 \mu \mathrm{m}$ lasers are used for this purpose. This correction takes into account slight machining or positioning inaccuracies and zero shifts of the angle drives. A check of this calibration using a single laser line is required anytime a grating is changed or when the grating controller loses track of its position after a memory reset (this is required due to occasional software errors in the controller). Any observed shift in grating driver step position can be subtracted from the output of the wavelength to grating angle conversion routine.

To check the linearity of the wavelength calibration, more spectral line markers are required. The transmittance of infrared transmission wavelength standard SRM 1921[GWH95], a polystyrene film, can provide many known spectral features. About 10 lines can be used as wavelength markers. The differences between the wavelengths given by the SRM certification and the wavelength peaks seen by the IR SCF are fitted versus the SRM wavelengths to a linear function. This fit result is used to correct the nominal wavelengths of the IR SCF. This procedure indicates spectral linearity to within $\pm 5 \mathrm{~nm}$.

Once the grating calibration is done, it is useful to verify the prism calibration. This is less critical than the grating calibration, since the prism bandpass is large compared to the grating bandpass. The prism calibration is done by fixing the grating at a wavelength and scanning the prism independently to find the location of its peak transmittance. After making this measurement at a number of grating positions, the prism calibration is found by fitting the peak position vs. wavelength. This calibration allows the prism peak to track the grating typically to within $10 \%$ of the prism bandpass, which maximizes the throughput of the spectrometer. The resulting calibration curve of wavelength versus prism encoder-driver step is shown in Figure 5.3. For reference, the useable ranges of the three gratings are also shown on the figure. The calibration routine allows for a shift in steps of the overall curve to be added to the output to correct for any errors in resetting the prism home position after memory reset of the driver control unit. In most cases (in the IR), this correction will not be needed because of the broad bandwidth of the prism relative to the slope of the step versus wavelength curve. The wavelength standard for the IR SCF is the $3.39 \mu \mathrm{m}$ HeNe laser which can calibrate the two IR gratings and the prism.

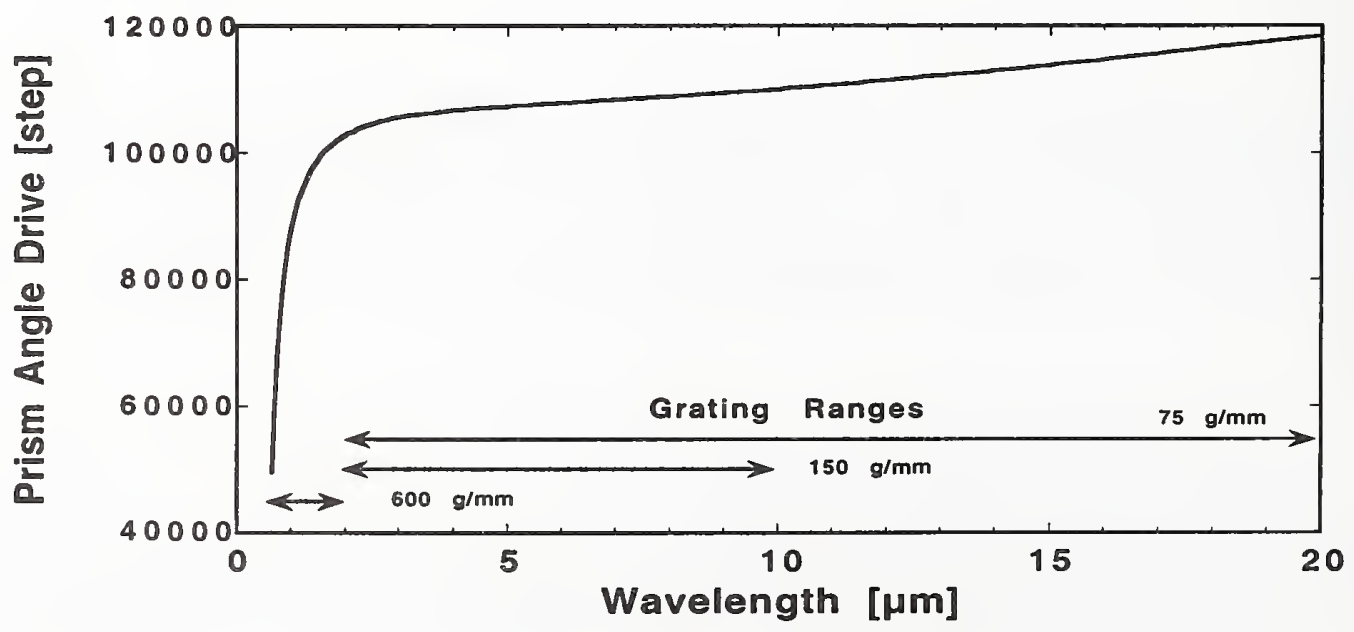


Figure 5.3 Prism calibration curve. The position (in encoder-driver steps) of peak prism transmittance versus wavelength. The spectral ranges of the 3 gratings are also indicated.

\subsubsection{Spectral Characterization}

The slit scatter function was measured for each of the gratings using $633 \mathrm{~nm}, 3.39 \mu \mathrm{m}$ and $10.6 \mu \mathrm{m}$ laser sources as appropriate. The profiles of the spectral line scans are given in terms of the full widths at $50 \%, 10 \%, 1 \%$, and $0.1 \%$ of the maximum value (see Table 5.1). Most results are presented for $1 \mathrm{~mm}$ entrance and exit apertures for the spectrometer, the normal configuration of the IR SCF spectrometer. Some of the spectral measurements were made with $2 \mathrm{~mm}$ entrance and exit apertures, so their values have been divided by 2 for comparison with the $1 \mathrm{~mm}$ aperture measurements and indicated by $(/ 2)$. The $600 \mathrm{~g} / \mathrm{mm}$ grating, which is not generally used for IR SCF measurements was measured using only a $633 \mathrm{~nm}$ laser input. The data presented in Table 5.1 may somewhat overestimate the true spectrometer linewidths, because both the $3.39 \mu \mathrm{m}$ and $10.6 \mu \mathrm{m}$ lasers may have been operating with some additional nearby weak lines also lasing. Some multiple peak structure was seen in the measured line wings. This affects the $1 \%$ and $0.1 \%$ widths more than the $50 \%$ and $10 \%$ full widths. This spectral width data can be useful in determining what responsivities would be observed at spectral resolutions other than that provided by the IR SCF. 
Table 5.1 Summary of monochromator spectral widths observed using laser sources. Entries followed by (/2) indicate measurements made using $2 \mathrm{~mm}$ entrance and exit apertures. All other entries were made with $1 \mathrm{~mm}$ apertures.

\begin{tabular}{|c|c|c|c|c|}
\hline & \multicolumn{3}{|c|}{ Wavelength [um] } & \\
\hline & 0.633 & 3.39 & 10.6 & \\
\hline Grating [g/mm] & \multicolumn{3}{|c|}{ Full Width [nm] at } & _\% Maximum \\
\hline 75 & & $\begin{array}{c}30(/ 2) \\
70(/ 2) \\
90(/ 2) \\
110(/ 2) \\
\end{array}$ & $\begin{array}{c}52(/ 2) \\
80(/ 2) \\
105(/ 2) \\
120(/ 2)\end{array}$ & $\begin{array}{c}50 \\
10 \\
1 \\
0.1 \\
\end{array}$ \\
\hline 150 & $\begin{array}{l}28 \\
47\end{array}$ & $\begin{array}{l}18(/ 2) \\
28(/ 2) \\
40(/ 2) \\
55(/ 2)\end{array}$ & $\begin{array}{l}20 \\
28 \\
55 \\
75 \\
\end{array}$ & $\begin{array}{c}50 \\
10 \\
1 \\
0.1 \\
\end{array}$ \\
\hline 600 & $\begin{array}{c}8 \\
17 \\
20\end{array}$ & & & $\begin{array}{c}50 \\
10 \\
1\end{array}$ \\
\hline
\end{tabular}

The demonstrated (see Figure 5.4) levels of rejection were 4.5 to 6.5 decades for the $75 \mathrm{~g} / \mathrm{mm}$ and $150 \mathrm{~g} / \mathrm{mm}$ gratings and $\approx 8$ decades for the $600 \mathrm{~g} / \mathrm{mm}$ grating. The lower levels of rejection in the IR are generally limited by thermal background noise or electrical system noise rather than by increased spectral scatter within the spectrometer.

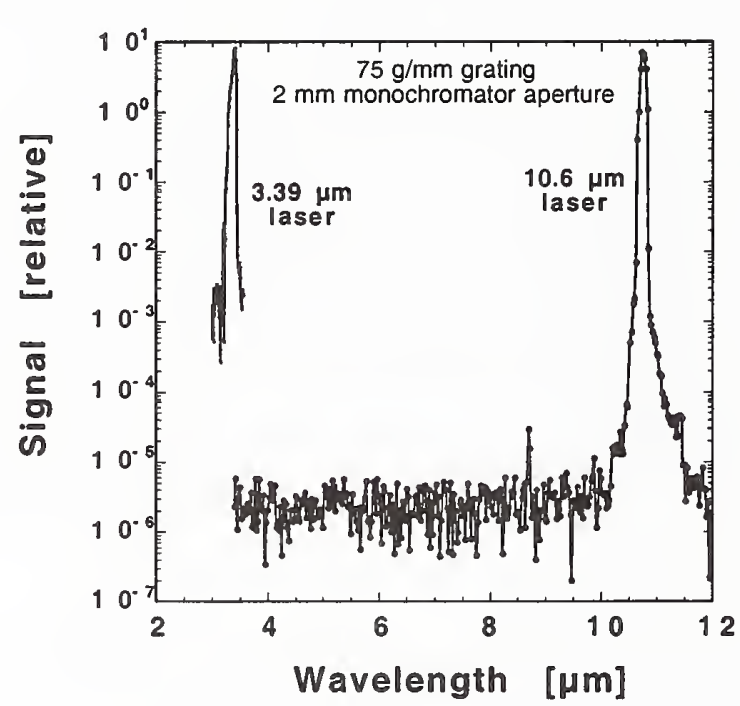

(a)

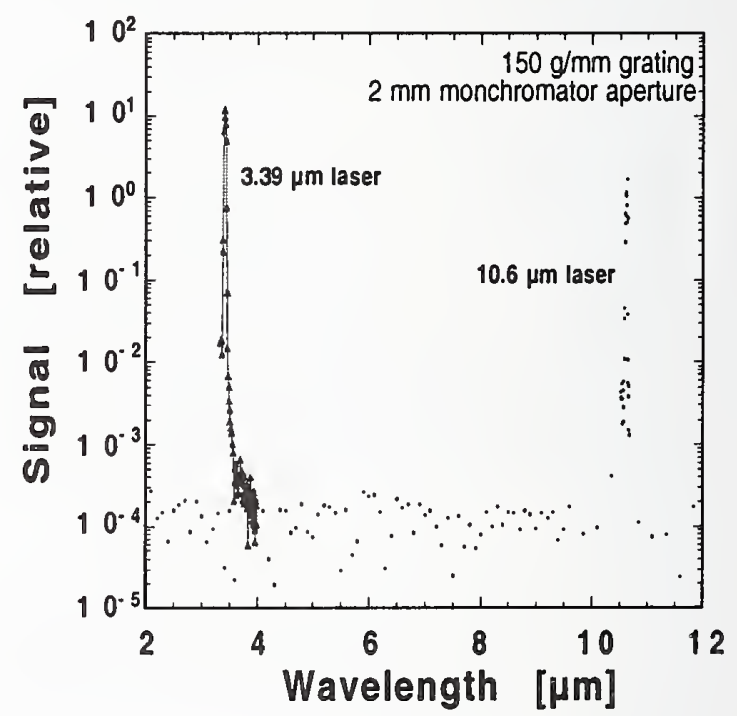

(b)

Figure 5.4 Spectral rejection is shown for a) the $75 \mathrm{~g} / \mathrm{mm}$ grating and b) the $150 \mathrm{~g} / \mathrm{mm}$ grating using two different lasers as sources. The baseline points in b) belong to the $10.6 \mu \mathrm{m}$ curve.

Second order rejection was tested for the $75 \mathrm{~g} / \mathrm{mm}$ grating using the $3.39 \mu \mathrm{m}$ laser as a source. Signal levels were compared for the spectrometer set to $3.39 \mu \mathrm{m}$ and to $6.8 \mu \mathrm{m}$. The second order $3.39 \mu \mathrm{m}$ signal seen at the $6.8 \mu \mathrm{m}$ setting was found to be reduced by a factor of 10 relative to first order $6.8 \mu \mathrm{m}$ signal. The $150 \mathrm{~g} / \mathrm{mm}$ grating exhibited $\approx 1.5$ decades rejection for the same setup. These results were extracted from spectral measurements of source radiance, detector 
efficiency, and spectrometer efficiency. These measurements only give the 2 nd order rejections for the specific wavelengths measured, other wavelengths will not necessarily have the same rejection levels. To quantify the size of this effect on detector spectral measurements, it is useful to compare measurements made with and without short wave cutoff filters. The size of any observed differences indicates the level of spectral impurities (i.e., out-of-band rejection).

\subsubsection{Temporal Characterization}

Temporal variations of the IR SCF output beam radiant power are attributed solely to source output variations [BRO77].

\subsubsection{Polarization Characterization}

The optical beam will have a significant degree of polarization. This results most obviously from the variation of the grating efficiency with polarization. The prism also contributes a polarization effect due to the large angle of incidence used. Because this variation has not been documented, when using polarization sensitive detectors, detector measurements should be made at two orthogonal detector orientations. For polarization independent detectors only a single orientation is required.

\subsubsection{Monitor Detectors}

The source amplitude is monitored by repeatedly swapping the standard detector and the detector under test into the spectrometer beam. Currently, a separate monitor detector which would allow for continuous normalization of source fluctuations is not used. The measurements depend on the short time source stability as described above. Future work will determine whether a monitor detector will be added.

\subsection{Electronics}

\subsubsection{Lock-in Output Gain}

The lock-in has a nominal output gain of 10 . That is, a $1 \mathrm{~V}$ rms input sinewave on the $1 \mathrm{~V}$ gain range produces a $10 \mathrm{~V}$ dc signal at the output of the lock-in. In addition, the ideal response of a sinewave lock-in amplifier to a squarewave input signal with an amplitude of unity is $\frac{4}{\pi \sqrt{2}}=0.90032$. The total nominal lock-in output gain for our squarewave optical signal is then $G_{\text {lockin }}=9.0032$. This gain was determined experimentally by using a dc optical signal and measuring the output of a detector/amplifier package directly with a dc DVM. The optical signal was then chopped and the lock-in output recorded. The ratio of the lock-in output to the dc measurement (a high accuracy measurement of the amplitude of the squarewave signal) is then a good measure of the actual overall lock-in gain. This measurement determined that the actual lock-in gain was $1.35 \% \pm 0.55 \%$ lower than the ideal value, so a correction factor of $C_{\text {lockin-output-gain }}=0.9865 \pm 0.0055$ must be used to multiply the lock-in output. 


\subsubsection{Lock-in Gain Range Calibration}

The lock-in gain ranges of $100 \mathrm{mV}, 10 \mathrm{mV}, 1 \mathrm{mV}$ and $0.1 \mathrm{mV}$ were calibrated electrically and referenced to the $1 \mathrm{~V}$ range. These factors $C_{\text {lockin-gain }}\left(G_{\text {lockin }}\right)$ given in Table 5.2 are multiplied by the measured voltage to give equivalent reading on the $1 \mathrm{~V}$ range.

Table 5.2 Lock-in Amplifier Correction Factors

\begin{tabular}{c|c|c}
$\begin{array}{c}\text { Lock-in } \\
\text { Gain Range }\end{array}$ & $\begin{array}{c}\text { Correction } \\
\text { factor } \\
C_{\text {lockin-gain }}\left(G_{\text {lockin }}\right)\end{array}$ & $\begin{array}{c}\text { Correction } \\
\text { factor } \\
\text { uncertainty }\end{array}$ \\
\hline $1 \mathrm{~V}$ & 1 & -- \\
$100 \mathrm{mV}$ & 0.99757 & 0.00008 \\
$10 \mathrm{mV}$ & 0.99736 & 0.00008 \\
$1 \mathrm{mV}$ & 0.99968 & 0.00022 \\
$0.1 \mathrm{mV}$ & 1.0032 & 0.0012
\end{tabular}

\subsubsection{Electronic System Linearity Check}

The linearity of the electronic portion of the measurement system was measured separately from the bolometer linearity. The results are discussed in reference EMC93.

\subsection{Transfer and Working Standards}

\subsubsection{Bolometer Standard}

\subsubsection{Responsivity vs. Time - Cryogenic Effects: cooldown, hold time, and refill}

Operation of the bolometer requires initially pumping out the dewar vacuum at room temperature to below $1 \times 10^{-4} \mathrm{~Pa}$ (as indicated by a gauge near the pumping station rather than near the dewar). The dewar's liquid $\mathrm{He}(\mathrm{LHe})$ and liquid $\mathrm{N}_{2}\left(\mathrm{LN}_{2}\right)$ reservoirs are then precooled with $\mathrm{LN}_{2}$ for about $1 \mathrm{~h}$. The $\mathrm{LN}_{2}$ is then removed from the LHe reservoir and replaced with LHe. A $6 \mathrm{~h}$ cooldown period is required for the bolometer to achieve stability to $\pm 0.25 \%$. The stability is achieved after a responsivity correction is applied (see following sections and Figure 5.8). This period of stability lasts $30 \mathrm{~h}$ before the LHe runs out. During this time, the $\mathrm{LN}_{2}$ reservoir must be refilled at $\approx 12 \mathrm{~h}$ intervals. The $30 \mathrm{~h}$ stable period may be extended if the LHe reservoir can be refilled before it is completely exhausted [EMG95].

\subsubsection{Responsivity vs. Frequency (including time dependence)}

For consistency, the bolometer responsivity is always given as a dc responsivity $R(\mathrm{dc})$. This value is determined by measuring the frequency response function, $R(f)$ and fitting it to a single time constant function: 


$$
R(f)=\frac{R(\mathrm{dc})}{\sqrt{1+\left(f / f_{3 \mathrm{~dB}}\right)^{2}}},
$$

where $f_{3 \mathrm{~dB}}$ is the $3 \mathrm{~dB}$ roll-off point. To convert from the observed ac signal to the dc equivalent level the correction factor

$$
C_{f_{\mathrm{3dB}}}(f)=\sqrt{1+\left(f / f_{3 \mathrm{~dB}}\right)^{2}}
$$

is required. The relative uncertainty of this correction is given by

$$
\frac{\Delta C_{f_{3 \mathrm{BB}}}(f)}{C_{f_{3 \mathrm{BB}}}(f)}=\frac{\sqrt{(\Delta f / f)^{2}+\left(\Delta f_{3 \mathrm{~dB}} / f_{3 \mathrm{~dB}}\right)^{2}}}{1+\left(f / f_{3 \mathrm{~dB}}\right)^{2}},
$$

where $\Delta f_{3 \mathrm{~dB}}$ and $\Delta f$ are the uncertainties of the bolometer $3 \mathrm{~dB}$ frequency point and the optical chopper frequency, respectively.

During the time the bolometer is operated at cryogenic temperature, $f_{3 \mathrm{~dB}}$ slowly shifts to lower frequency. This is due to residual gas within the dewar freezing out onto the bolometer. This increases the bolometer thermal mass and reduces the frequency of $f_{3 \mathrm{~dB}}$. This is a repeatable effect. When the bolometer is warmed to room temperature and repumped, $f_{3 \mathrm{~dB}}$ will return to its nominal starting value of around $85 \mathrm{~Hz}$. (The bolometer is generally continuously pumped when it is not in use at room temperature.) Rates of decrease of $f_{3 \mathrm{~dB}}$ have been observed in the range of 0.06 to $0.3 \mathrm{~Hz} / \mathrm{h}$. The variation of the decay rate probably reflects the quality of the initial pump down. Rates larger than the high end of this are indicative of a vacuum problem such as an air leak, which needs to be corrected before the bolometer can be used reliably. Measurement of $f_{3 \mathrm{~dB}}$ is required at least twice during the stable operation period of the bolometer to allow for proper correction of the bolometer responsivity. Figure 5.5 shows an example of the drift of $f_{3 \mathrm{~dB}}$ with time. The times of the initial cryogen fill and a He refill are also indicated. Liquid $\mathrm{N}_{2}$ is added twice per day with minimal effect on the bolometer (not shown in the figure). The scatter at the beginnings of each data set indicates a slight change in the bolometer equilibrium as the bolometer operation is switched between operating modes (see sec. 5.3.1). The low point at the end of the second cluster of points is due to the cryogen running out. 


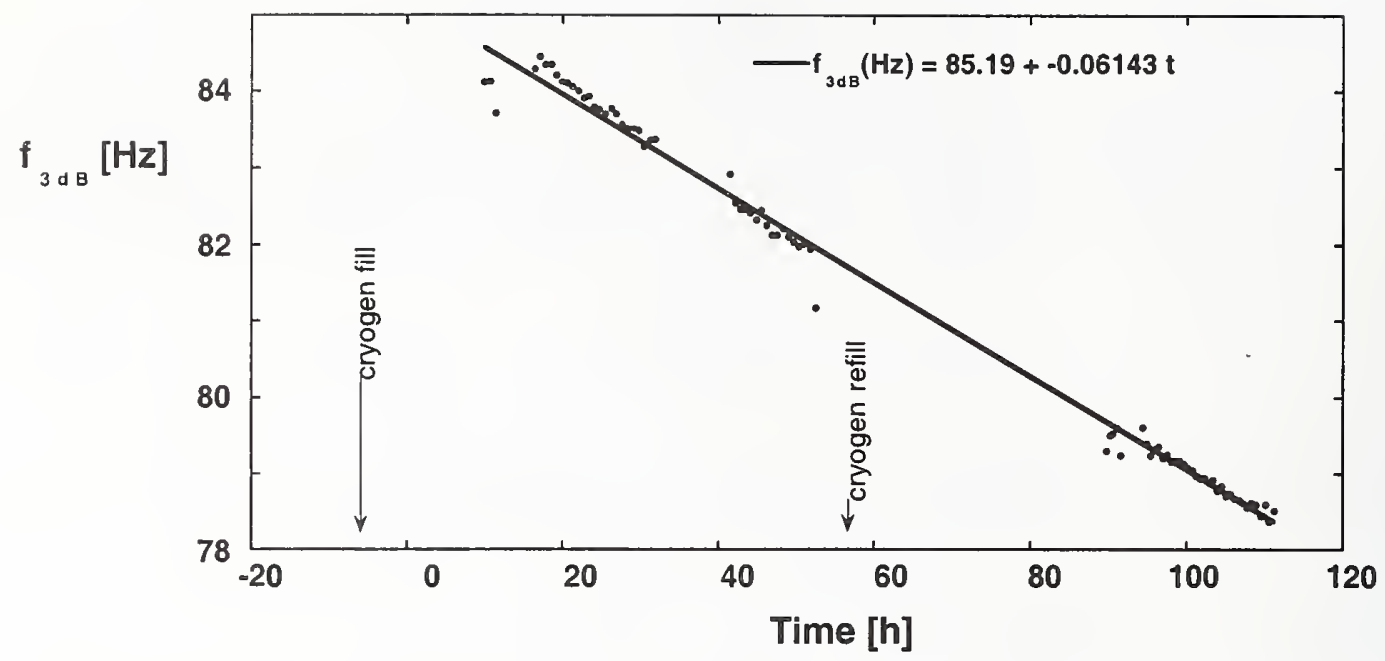

Figure 5.5 Change of $3 \mathrm{~dB}$ roll-off frequency with time. The line indicates the linear fit to the data.

\subsubsection{Responsivity vs. Temperature}

The responsivity of the bolometer depends on its quiescent temperature. Because a number of effects can cause bolometer temperature variations (such as IR background loading and even atmospheric pressure variations changing the LHe boiling point) it is important to monitor and correct for the bolometer temperature. It is this adjustment for temperature dependent responsivity variations that allow the bolometer to be used as a stable transfer standard. These temperature dependent changes are monitored by determining the voltage drop across the bolometer, $V_{\mathrm{d}} . V_{\mathrm{d}}$ is found by recording the voltage after the JFET impedance transformer (at the point labeled dc OUT of Figure 4.2) with the bolometer bias voltage on and off, rather than reading $V_{\mathrm{d}}$ directly at the bolometer which has a high impedance at operating temperature. The bias voltage is switched under computer control with a $50 \mathrm{~s}$ wait time after switching to allow the voltage to settle before reading with a DVM [EMG95]. The correction factor, $C_{V_{d}}$ for operating the bolometer with $V_{\mathrm{d}}$ at levels other than the nominal level used at calibration $\left(V_{\mathrm{d}}(\mathrm{cal})=0.75 \mathrm{~V}\right)$ is given by:

$$
C_{V_{\mathrm{d}}}=\frac{1}{1+\frac{M \cdot\left(V_{\mathrm{d}}-V_{\mathrm{d}}(\mathrm{cal})\right)}{V_{\text {out }}(\mathrm{cal})}},
$$

where $M(=7.7901 \pm 0.004)$ is the linear variation of output signal versus $V_{\mathrm{d}}$, and $V_{\text {out }}$ (cal) $(=4.3986 \pm 0.003 \mathrm{~V})$ is the output signal at calibration. The variation was determined by measuring the bolometer output voltage with constant optical input as the bolometer operating temperature was varied. The temperature was changed by varying the pressure in the $\mathrm{LHe}$ reservoir and thus the LHe boiling point. The uncertainty of this correction is given by 


$$
\frac{\Delta C_{V_{\mathrm{d}}}}{C_{V_{\mathrm{d}}}}=\frac{\sqrt{\left(\frac{\Delta M \cdot\left(V_{\mathrm{d}}-V_{\mathrm{d}}(\mathrm{cal})\right)}{V_{\text {out }}(\mathrm{cal})}\right)^{2}+\left(\frac{M \cdot \Delta V_{\mathrm{d}}}{V_{\text {out }}(\mathrm{cal})}\right)^{2}+\left(\frac{M \cdot\left(V_{\mathrm{d}}-V_{\mathrm{d}}(\mathrm{cal})\right) \Delta V_{\text {out }}(\mathrm{cal})}{\left(V_{\text {out }}(\mathrm{cal})\right)^{2}}\right)^{2}}}{1+\frac{M \cdot\left(V_{\mathrm{d}}-V_{\mathrm{d}}(\mathrm{cal})\right)}{V_{\text {out }}(\mathrm{cal})}},
$$

where $\Delta M, \Delta V_{\mathrm{d}}$, and $\Delta V_{\text {out }}$ (cal) are the uncertainties of $M, V_{\mathrm{d}}$ and $V_{\text {out }}(\mathrm{cal})$, respectively. For further discussion of this correction, see Eppeldauer, et al. [EMG95].

\subsubsection{Responsivity vs. Bias Voltage}

The bolometer responsivity is linearly dependent on the bias voltage $V_{\text {bias }}$. To remove this effect, the responsivity is corrected to a nominal value of $V_{\text {bias }}(\mathrm{cal})=15.1 \mathrm{~V}$. Because the bias supply is a stabilized precision circuit, variations of this value are typically very small, less than $2 \mathrm{mV}$ or $\approx 0.01 \%$. This correction factor for the bolometer responsivity is given as:

$$
C_{V_{\text {bias }}}=\frac{V_{\text {bias }}(\text { cal })}{V_{\text {bias }}(\text { actual })} \text {, }
$$

where $V_{\text {bias }}$ (actual) is the actual bias voltage during the time of measurement. Because the bias is stabilized to such relatively high precision the uncertainty of this correction can be neglected.

\subsubsection{Responsivity Nonlinearity}

The nonlinearity of the bolometer responsivity was found by measuring the transmittance of a nominally $10 \%$ transmitting neutral density filter as the input optical power was varied. Because the filter transmittance should not depend on optical power at these low levels $(<10 \mu \mathrm{W})$ any transmittance variation must result from nonlinearity of the system. To determine whether this nonlinearity was due to the bolometer or the rest of the measurement electronics, the nonlinearity of the entire measurement system was compared to that of the system with the bolometer excluded. This was done by substituting a Si diode, known to be highly linear, for the bolometer and measuring the nonlinearity again as described above. The component not attributable to the bolometer was found to be below $0.1 \%$ [EMC93] (Additionally, the bolometer amplifier nonlinearity was measured electronically and found also to be below $0.1 \%$.). The transmittance nonlinearity was fit to a quadratic form. Because the use of a $\approx 10 \%$ transmitting filter only measures the nonlinearity over a single decade, a small additional correction to the nonlinearity was made. This was done by taking the product of the nonlinearity at a level of $x$ and the nonlinearity at a level of $0.1 \mathrm{x}$. This "two decade" nonlinearity allows the signal to be corrected to within $0.1 \%$ of the true value. The form of the nonlinearity correction factor is $C_{\mathrm{NL}}=1+n_{1} V+n_{2} V^{2}$, where $n_{1}$ and $n_{2}$ are fit coefficients and $V$ is the voltage out of the bolometer amplifier at a gain of $\times 20$. (This correction is referenced to the $\times 20$ gain level rather than the $\mathrm{x} 100$ level used for the rest of the calibration because the nonlinearity correction is generally only significant on the $\mathrm{x} 20$ gain setting.) The nonlinearity fit parameters were found to depend on the bolometer temperature so they were in turn measured and fit with a linear function vs. $V_{\mathrm{d}}$. The resulting fit parameters are given as: 


$$
\begin{aligned}
& n_{1}=0.0231-0.01237 V_{d} \\
& n_{2}=0.000909-0.0005246 V_{d},
\end{aligned}
$$

with uncertainties of $\Delta n_{1}=0.001$ and $\Delta n_{2}=0.00005$. Although the nonlinearity also depends on $V_{\mathrm{d}}$, it ranges up to $\approx 10 \%$ at $\approx 10 \mu \mathrm{W}$. Values of $C_{\mathrm{NL}}$, as determined by these fit parameters versus $V$ are shown for several values of $V_{d}$ in Figure 5.6. (For reference, the $20 \%$ nonlinearity of the $V_{\mathrm{d}}=0.6 \mathrm{~V}$ curve, at a signal level of $10 \mathrm{~V}$, corresponds to $\approx 40 \mu \mathrm{W}$. The $10 \mathrm{~V}$ point on the $V_{\mathrm{d}}=1.6 \mathrm{~V}$ curve corresponds to $\approx 10 \mu \mathrm{W}$.) The uncertainty of this correction factor is given by

$$
\Delta C_{\mathrm{NL}}=\sqrt{\left(V \Delta n_{1}\right)^{2}+\left(V \Delta n_{1}\right)^{2}} .
$$

(Note: $V$ is bolometer output on the gain 20x range). In this case the relative and absolute uncertainties are nearly equal because the correction factor is always near one.)

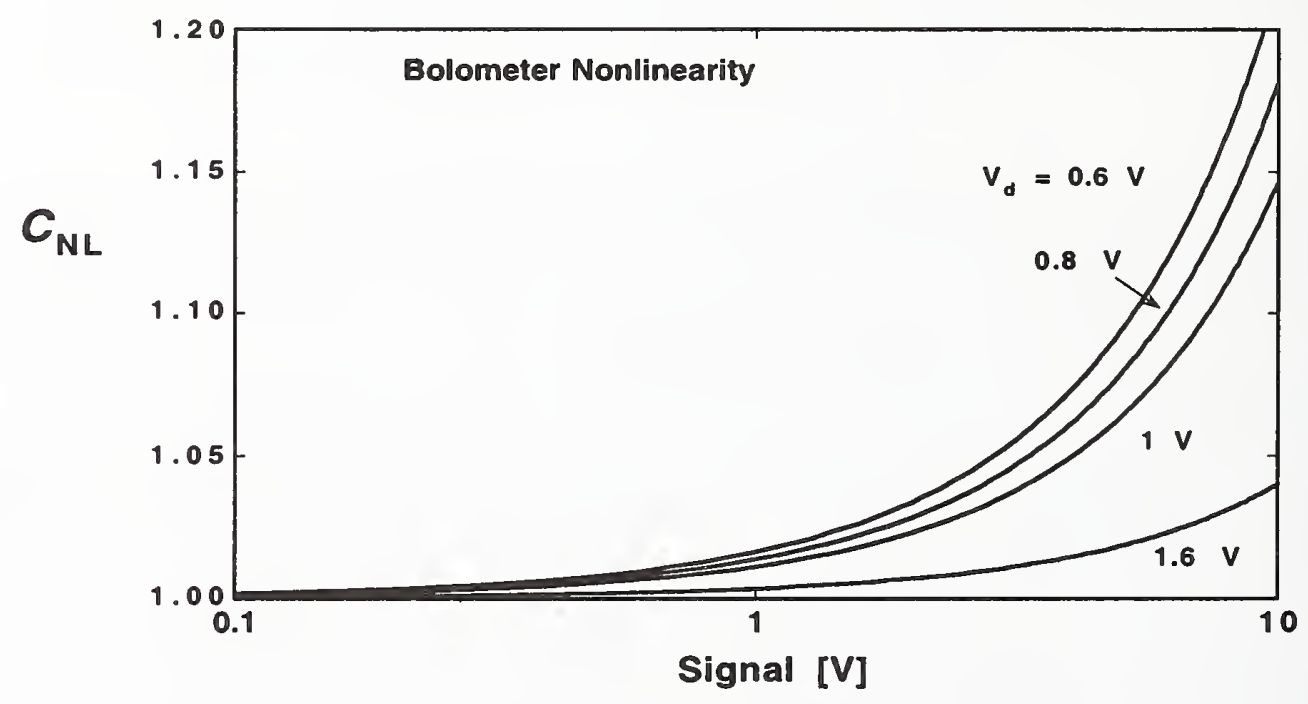

Figure 5.6 Bolometer nonlinearity correction is shown versus (lockin) output signal level on bolometer gain range $\mathrm{x} 20$ for various voltage drops across the bolometer.

\subsubsection{Dynamic Range}

The noise equivalent power of the bolometer was measured to be $20 \mathrm{pW}$ with a $0.3 \mathrm{~Hz}$ measurement bandwidth [EMC94]. This is equivalent to a noise density of $36 \mathrm{pW} / \sqrt{\mathrm{Hz}}$. This noise limit compared with the nonlinearity limit mentioned in the previous section of $\approx 10 \mu \mathrm{W}$ gives a dynamic range of $5 \times 10^{5}$. 


\subsubsection{Spatial Responsivity}

The spatial variation of the bolometer was measured by scanning the bolometer across the imaged output of the monochromator. An example of a spatial scan is shown in Figure 5.7, indicating a $2.3 \mathrm{~mm}$ region where the responsivity is constant to within $\pm 0.7 \%$. By proper alignment, it should be possible to use only the central region of the bolometer resulting in an uncertainty of only $0.3 \%$. The diameter of the optical beam is $\approx 1 \mathrm{~mm}$ as can be seen from the width of the transition region. The width of the flat responsivity region plus the width of the beam adds to $3.3 \mathrm{~mm}$ which is consistent with expectations, as the bolometer aperture is $3.5 \mathrm{~mm}$. For a detailed discussion of these spatial variations of responsivity see Eppeldauer et al. [EMC93].

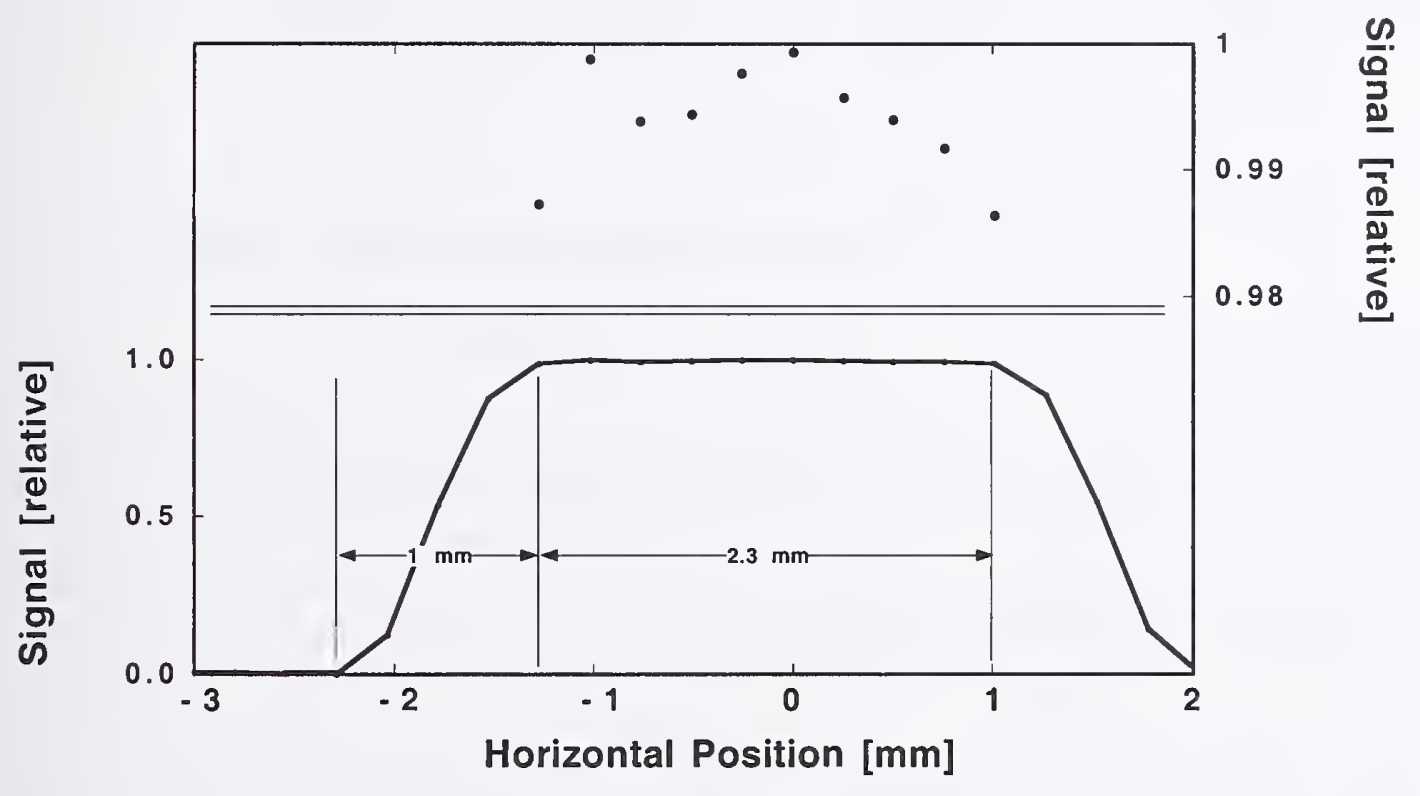

Figure 5.7 Bolometer spatial responsivity. A $1 \mathrm{~mm}$ beam is scanned across the bolometer. The points above the double line use the expanded scale on the right to better view the variations of the plateau region.

\subsubsection{Window Properties}

To maintain the optical quality of the $\mathrm{KBr}$ window, it must be kept dry. When the system is not in use, a cover containing desiccant is installed. This desiccant is checked every 2 weeks and replaced as necessary. When making measurements, a shroud purged with dry air is used. A supply of spare windows from the same manufacturer's lot is available to allow for replacement if accidental degradation of the surface should occur. It is possible to maintain the window quality over a period of months. 


\subsubsection{Bolometer Gain Range Calibration}

The gain calibration of the bolometer amplifier was measured and is shown in Table 5.3 referenced to gain 100 , the most commonly used bolometer gain.

Table 5.3 Bolometer ac amplifier gain calibration referenced to gain x100

\begin{tabular}{c|c|c|c} 
& $\begin{array}{c}\text { Bolometer } \\
\text { Amplifier } \\
\text { Gain Range }\end{array}$ & $\begin{array}{c}\text { Conversion } \\
\text { Factor to } \\
\text { Gain x100 }\end{array}$ & Uncertainty \\
\hline$G_{20 \rightarrow 100}$ & $\mathrm{x} 20$ & 4.96686 & 0.00184 \\
$G_{100 \rightarrow 100}$ & $\mathrm{x} 100$ & 1 & - \\
$G_{200 \rightarrow 100}$ & $\mathrm{x} 200$ & 0.49299 & 0.00014 \\
$G_{1000 \rightarrow 100}$ & $\mathrm{x} 1000$ & 0.09942 & 0.00002
\end{tabular}

\subsubsection{Overall Bolometer Responsivity Correction Equation}

The total correction equation for the bolometer output, corrected to gain $\mathrm{x} 100$, is:

$$
C_{\mathrm{Tot}}=C_{f_{\mathrm{sdB}}}(f) \cdot C_{V_{\mathrm{d}}} \cdot C_{V_{\text {bias }}} \cdot G_{x \rightarrow 100} \cdot C_{\mathrm{NL}}\left(V ; V_{\mathrm{d}}\right) \cdot C_{\text {lockin-output-gain }} \cdot C_{\text {lockin-gain }}\left(G_{\text {lockin }}\right) .
$$

The relative uncertainty of this total correction is given by:

$$
\frac{\Delta C_{\mathrm{Tot}}}{C_{\mathrm{Tot}}}=\sqrt{\left(\frac{\Delta C_{f_{\mathrm{saB}}}(f)}{C_{f_{3 \mathrm{~B}}}(f)}\right)^{2}+\left(\frac{\Delta C_{V_{\mathrm{d}}}}{C_{V_{\mathrm{d}}}}\right)^{2}+\left(\frac{\Delta G_{x \rightarrow 100}}{G_{x \rightarrow 100}}\right)^{2}+\left(\frac{\Delta C_{\mathrm{NL}}}{C_{\mathrm{NL}}}\right)^{2}+\left(\frac{\Delta C_{\text {lockin-output-gain }}}{C_{\text {lockin-output-gain }}}\right)^{2}+\left(\frac{\Delta C_{\text {lockin-gain }}}{C_{\text {lockin-gain }}}\right)^{2}}
$$

(A similar term due to $\Delta C_{V_{\text {bis }}}$ was not included because of its negligible size.) Figure 5.8 shows the effect of applying this total correction to the raw output signal of the bolometer, which had an optical input beam with constant power. After an initial $\approx 6 \mathrm{~h}$ cool down period, the corrected signal shows stability to $\approx \pm 0.25 \%$ for $30 \mathrm{hr}$, after which the bolometer cryogen (LHe) was used up. The peaks in the raw signal at $7 \mathrm{~h}, 24 \mathrm{~h}$, and $30 \mathrm{~h}$ are the result of $\mathrm{LN}_{2}$ refills. These variations are removed by the correction process. 


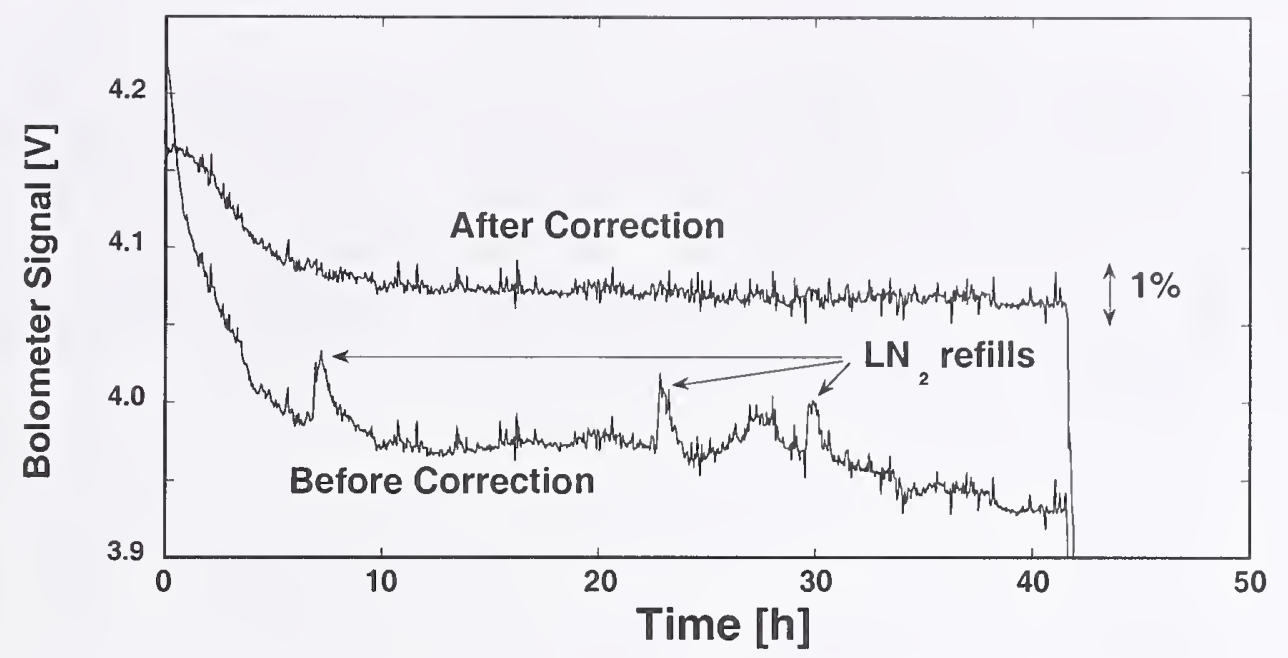

Figure 5.8 Effect of Bolometer Correction Procedure.

\subsubsection{InSb Working Standards}

\subsubsection{Responsivity vs. Time}

A $0.25 \%$ responsivity change was measured during the $15.5 \mathrm{~h}$ long hold time of the dewar (see Figure 5.9). This hold time depends on the dewar vacuum level. Immediately after repumping the dewar, hold times in excess of $24 \mathrm{~h}$ are possible. The $0.1 \%$ instability in the second half of Figure 5.9 was measured from 6 p.m. when the environment of the setup was undisturbed. The radiation source was the quartz-halogen lamp, operated at half of the nominal $100 \mathrm{~W}$ power. The low temperature of the source and the $2 \mu \mathrm{m}$ cut-on (order sorting) filter was required to maintain the stability to within $0.1 \%$. Radiation shorter than $\approx 2 \mu \mathrm{m}$ produces a responsivity fluctuation due to trapped charges accumulating within the detector. The variations are seen as sudden jumps in the output level. This effect is referred to as "flashing." Flashing can reach the few percent level if the visible to near infrared radiation is not eliminated from the detector.

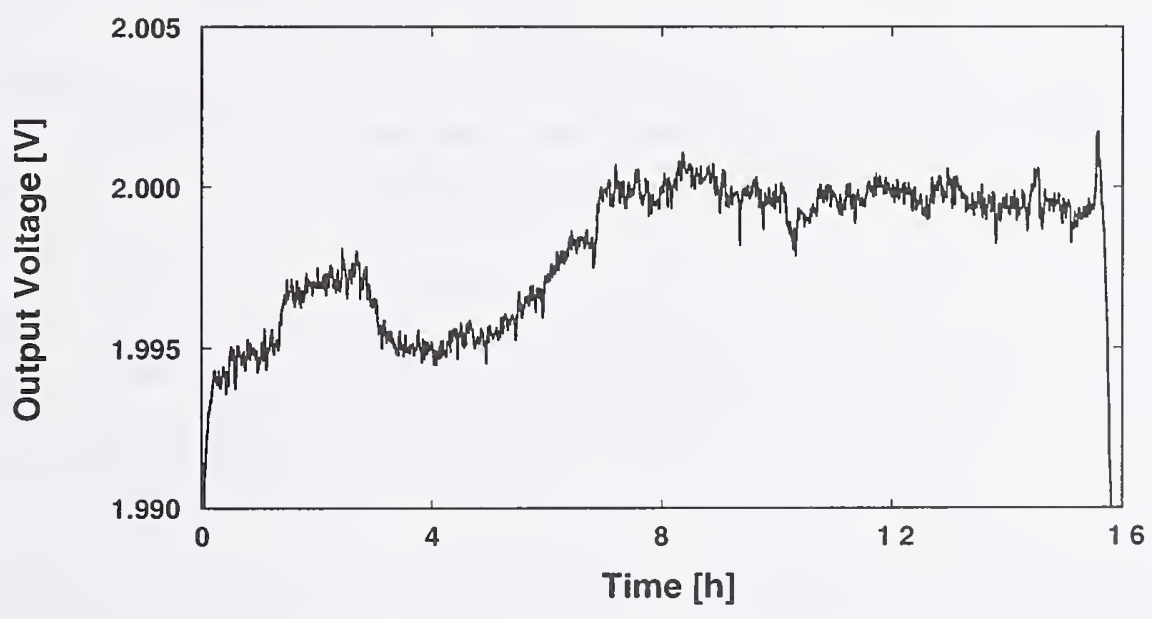

Figure 5.9 Test of InSb stability vs. time at $3.8 \mu \mathrm{m}$. 


\subsubsection{Responsivity vs. Frequency}

The current preamplifiers were optimized for a chopping frequency of $40 \mathrm{~Hz}$. Full and partial frequency compensations were used for the different gains. As a result, the $3 \mathrm{~dB}$ roll-off points of the different signal gains were tuned to $400 \mathrm{~Hz}$ or higher. At the same time, the voltage gain was kept equal to or less than $10 \mathrm{x}$ at $40 \mathrm{~Hz}$. Also, the loop gains at $40 \mathrm{~Hz}$ were higher than $10000 \mathrm{x}$ for all signal gains, resulting in high accuracy in the current-to-voltage conversion. The measured and normalized frequency dependent signal responsivities at gains $10^{4}, 10^{5}$, and $10^{6} \mathrm{~V} / \mathrm{A}$ are shown in Figure 5.10.

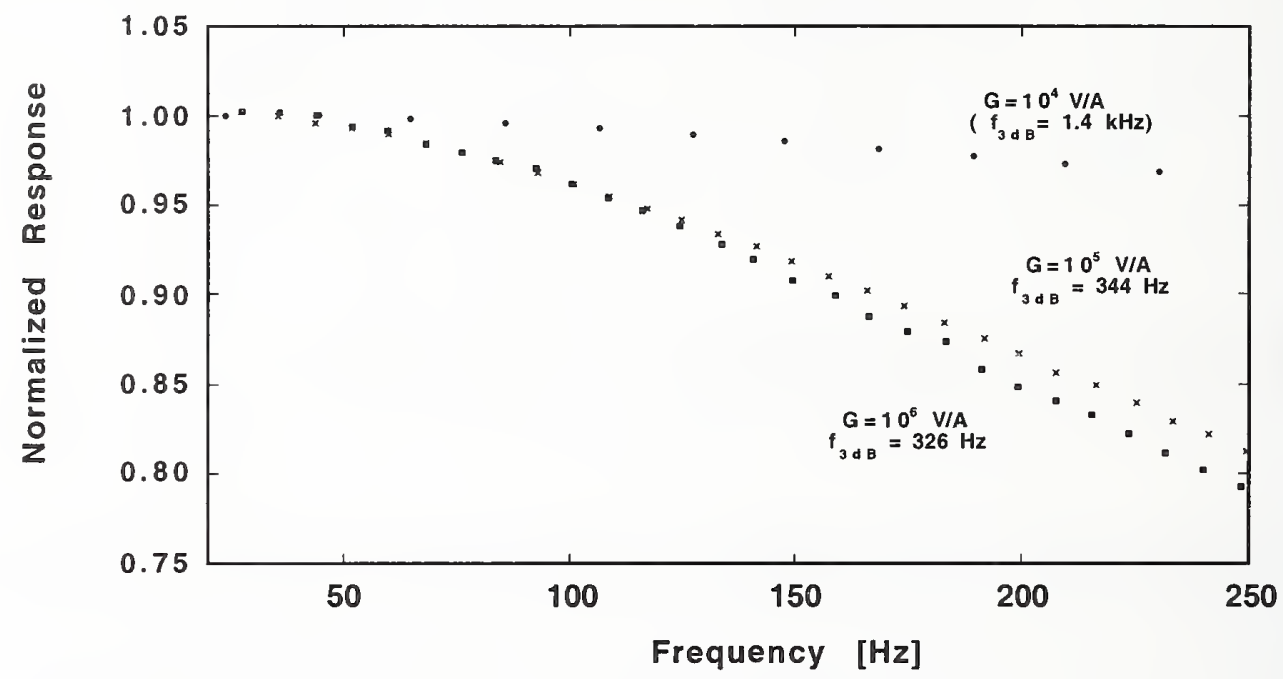

Figure 5.10 InSb working standard radiometer responsivity vs. frequency normalized to unity at dc.

\subsubsection{Responsivity Nonlinearity}

The nonlinearity of the InSb radiometer was tested by comparison to a calibrated transfer standard Ge detector over a range of input power levels. The measurements were made using the chopped beam of a laser diode at $1.3 \mu \mathrm{m}$ where the two detectors spectrally overlap. ND filters were inserted into the beam to vary the radiant power level as the two detectors were substituted for each other and the InSb to Ge responsivity ratios determined. 


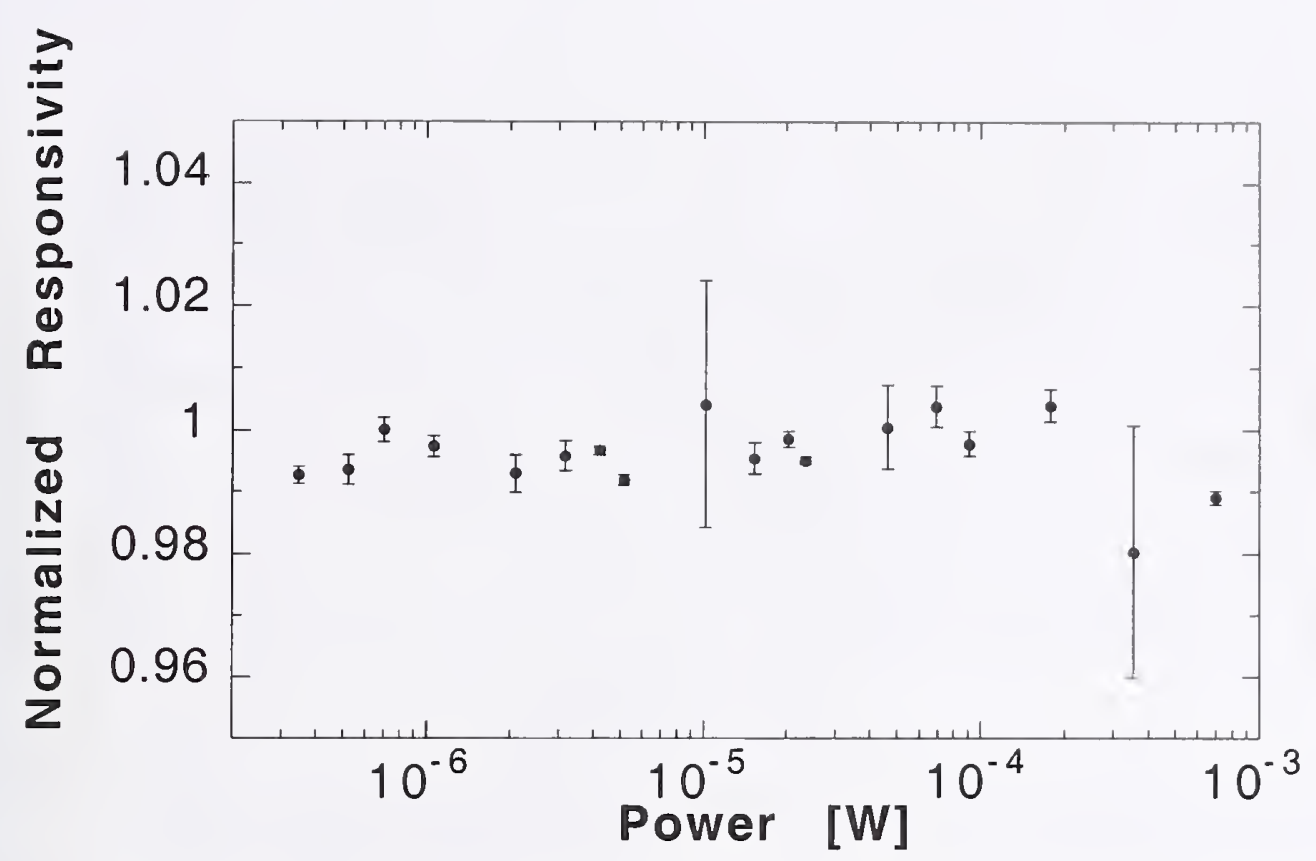

Figure 5.11 Ratios of InSb to Ge responsivity (for identical radiant power inputs) divided by a constant to yield a mean value near unity.

The responsivity ratios, which have all been divided by 0.338 to yield a mean value near unity (for ease of viewing relative deviations), are shown in Figure 5.11. While the current-to-voltage conversion uncertainties were about $0.1 \%$, the uncertainties of the lock-in amplifier gain calibrations were in the order of $0.5 \%$. The InSb flashing also contributed significantly to the uncertainty of these measurements. The relative standard uncertainties were calculated for each of the ratio measurements. The dynamic range of the linearity test was limited by the poor signal-to-noise ratio at low power levels. The $\mathrm{InSb}$ detector is linear to about $0.1 \mathrm{~mW}$. At $0.75 \mathrm{~mW}$ the responsivity decrease is roughly $1 \%$. The estimated overall relative standard uncertainty of these linearity tests is about $1 \%$. The measured responsivity decrease at the high power end is within the range expected for these detectors.

\subsubsection{Dynamic Range - Noise Floor}

The noise equivalent detector current was measured versus wavelength using the IR SCF monochromator. The background radiation was chopped at $43 \mathrm{~Hz}$ (i.e., the arc source was off) producing the detector mean current as shown in Figure 5.12. The background current noise increased by more than a factor of two, as the monochromator was tuned from $2 \mu \mathrm{m}$ to $5 \mu \mathrm{m}$. The detector current noise was $\approx 30$ times smaller than the mean background produced detector current at $5 \mu \mathrm{m}$. This detector current noise determines the limiting noise equivalent power (NEP) of the InSb radiometer. The NEP at the peak responsivity was $0.5 \mathrm{pW}$ in an electrical bandwidth of $0.3 \mathrm{~Hz}$. The ratio of the dc ambient background current to the current noise (both are detector currents) was $10^{7}$. 


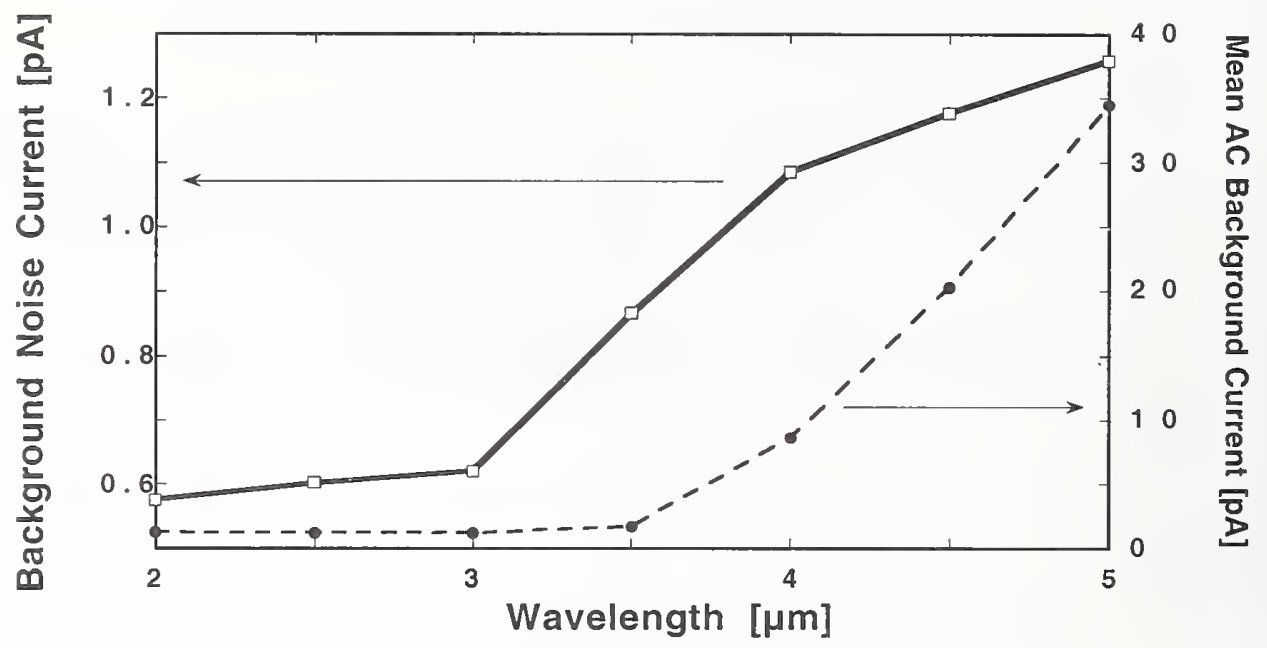

Figure 5.12 Noise and background current vs. monochromator wavelength.

The maximum detector current can be measured at a gain of $10^{4} \mathrm{~V} / \mathrm{A}$. A $0.1 \mathrm{~mA}$ maximum current on the $10 \mathrm{~V}$ full scale output voltage corresponds to a maximum measurable radiant power of $50 \mu \mathrm{W}$ at the InSb peak responsivity.

\subsubsection{Spatial/Angular Responsivity}

The spatial responsivity changes were measured along the horizontal and vertical diameters of the InSb detectors. Figure 5.13a shows the horizontal scan within the $3.5 \mathrm{~mm}$ diameter of the precision aperture. The measured peak change was about $1 \%$. Vertical scans were similar. The angular responsivity was tested by rotating the detector around its vertical axis. The axis of rotation was close to the center of the precision aperture. The measurement results shown in Figure $5.13 \mathrm{~b}$ were made using a point source $1.2 \mathrm{~m}$ from the detector. The measured field of view is $17^{\circ}$. The solid curve on the figure is the expected Lambertian cosine function. The maximum deviation of the measured angular responsivity from a cosine form is $\pm 1 \%$. 


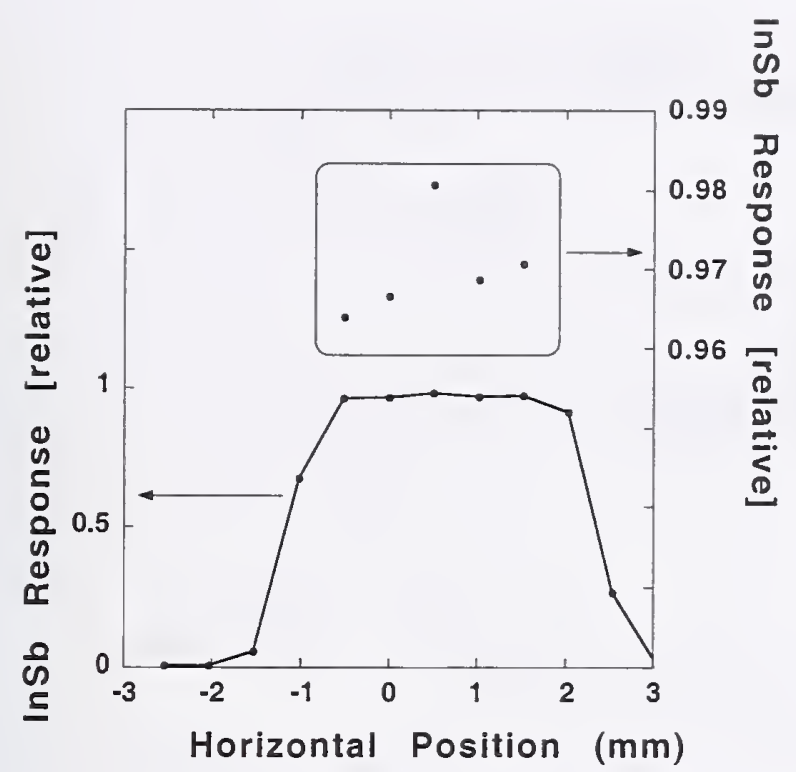

(a)

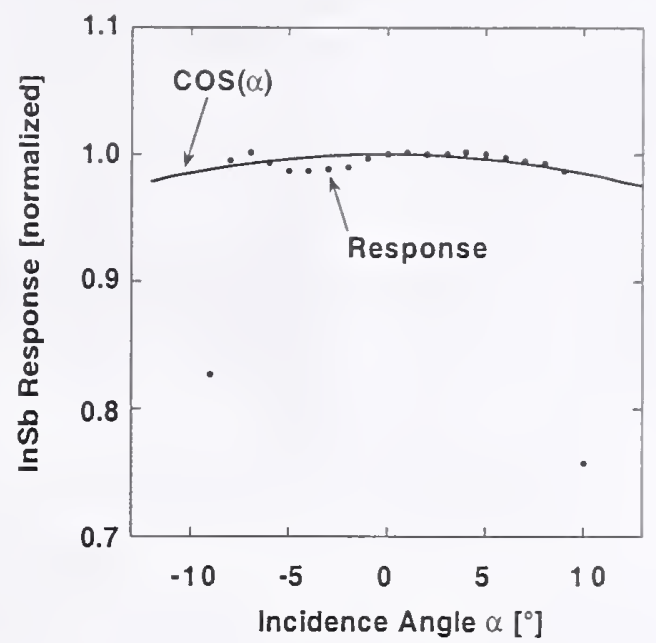

(b)

Figure 5.13 a) InSb horizontal spatial responsivity variation, right side shows expanded scale. b) InSb angular responsivity variation.

\section{Spectral Radiant Power Responsivity Scale Realization}

\subsection{Bolometer Responsivity Scale}

\subsubsection{Relative Spectral Responsivity}

Because the bolometer should respond only to absorbed radiation, its relative spectral responsivity is simply the product of the absorptance of the bolometer coating and the transmittance of the bolometer window.

\subsubsection{Bolometer Spectral Absorptance}

The spectral absorptance of the bolometer gold black coating was measured using a FTIR spectrometer system with hemispherical collection optics. This allowed the total reflectance (diffuse and specular) to be measured. Two additional measurements were made using laser sources. At $0.633 \mu \mathrm{m}$ and $10.6 \mu \mathrm{m}$, the reflectances were $1.2 \pm 0.3 \%$ and $0.5 \pm 0.3 \%$, respectively. The results are shown in Figure 6.1. A linear fit to the FTIR data from $2 \mu \mathrm{m}$ to $17 \mu \mathrm{m}$ yielded a minimal slope with a magnitude not much larger than its uncertainty. Because of this, the FTIR reflectance data was simply averaged yielding a wavelength independent $\mathrm{R}=$ $0.75 \%$. This is roughly consistent with the values and uncertainties of the two laser reflectance measurements. As the uncertainty of the absolute value of the FTIR measurements is difficult to ascertain, the uncertainty of the average (which is nearly the same as the average of the laser results) is assigned the value of the laser measurements, $0.3 \%$. Because the IR termination of the reflectance data beyond $17 \mu \mathrm{m}$ was due to low FTIR sensitivity rather than a variation of gold black, it is expected that an extrapolation of this data will be valid to at least $20 \mu \mathrm{m}$. 


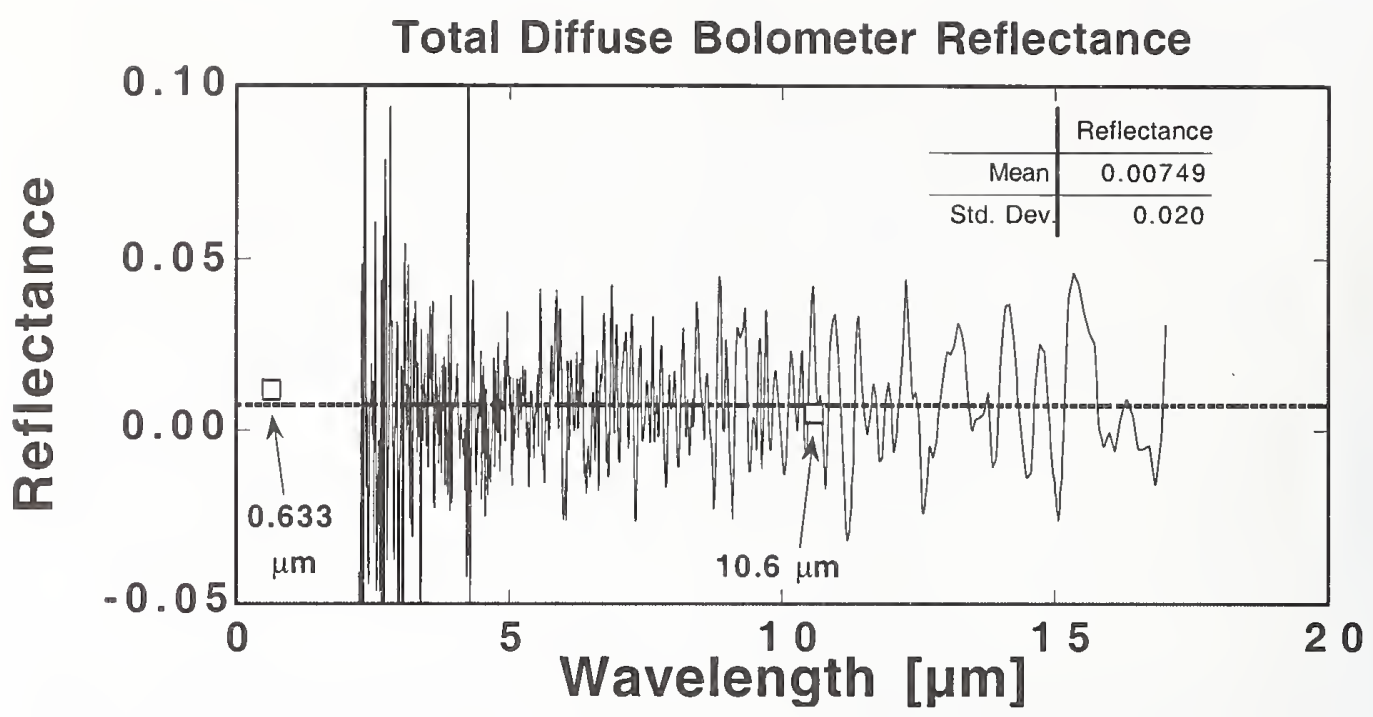

Figure 6.1 The solid curve shows the bolometer gold black reflectance measured using a FTIR spectrometer. The reflectance was also measured with laser sources as indicated by the two open squares at $0.633 \mu \mathrm{m}$ and $10.6 \mu \mathrm{m}$.

\subsubsection{Bolometer Window Spectral Transmittance}

The transmittance of the bolometer window was measured from $600 \mathrm{~nm}$ to beyond $18 \mu \mathrm{m}$. To accomplish this, several measurement systems were used: the IR SCF itself, the Vis/NIR SCF, an FTIR spectrometer, as well as measurements made using laser sources. This allowed for consistency checks of the results. One difficulty in these measurements was caused by the deflection of the transmitted beam due to the window wedge angle. Each measurement system handled this problem differently.

The IR SCF was used to measure single $\mathrm{KBr}$ window samples from $2 \mu \mathrm{m}$ to $20 \mu \mathrm{m}$. Here the deflection of the transmitted beam was handled by repositioning the detector for maximum signal for the window in and out of beam measurements. This should reduce the uncertainty due to detector spatial nonuniformity to equal or less than the repeatability uncertainty of the measurements. The transmittance is shown in Figure 6.2a with the error bars being the standard deviation of 5 to 9 repeated measurements. The mean size of the error bars is $\approx 0.1 \%$ for the region from $2 \mu \mathrm{m}$ to $16 \mu \mathrm{m}$. The IR SCF was used to measure the transmittance difference of two window samples (see Figure 6.2b). The mean difference between the two measurements was $0.016 \%$ with a standard deviation of $0.2 \%$. These small differences allow for window interchanges with the standard deviation used as the uncertainty. 


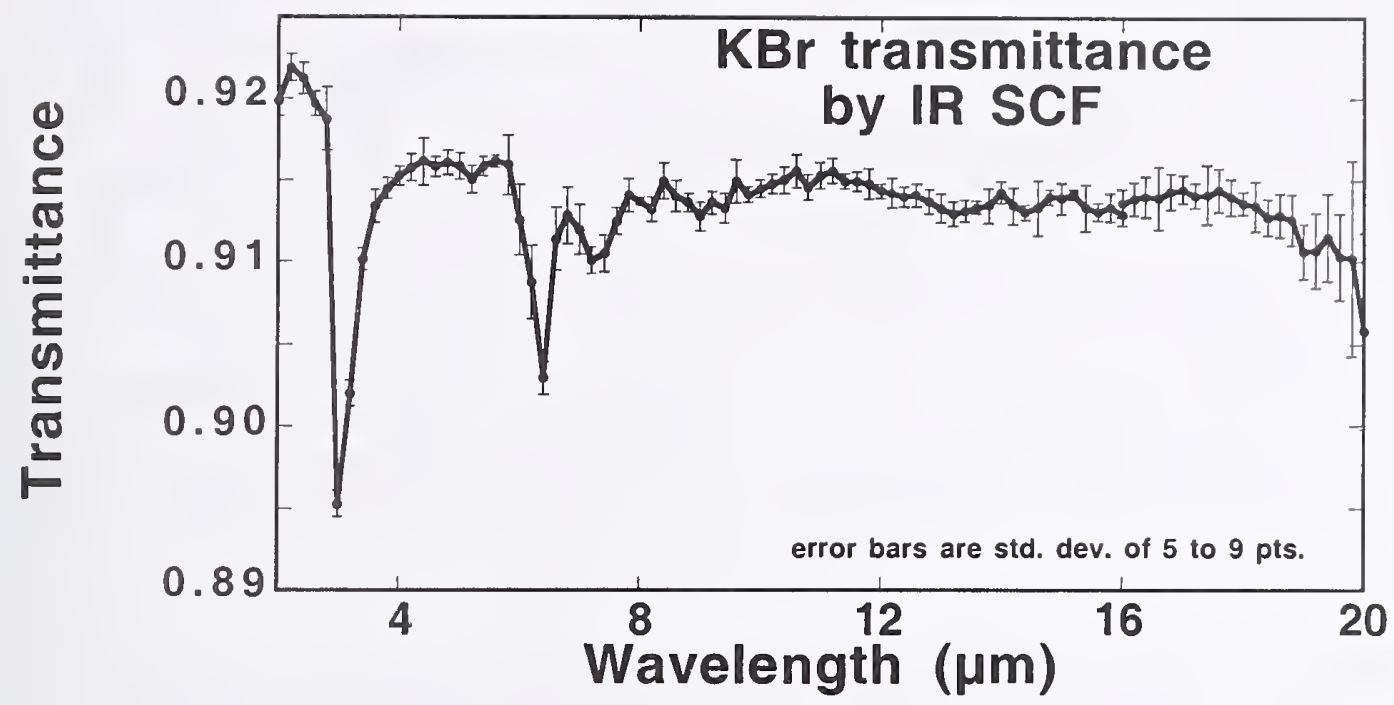

(a)

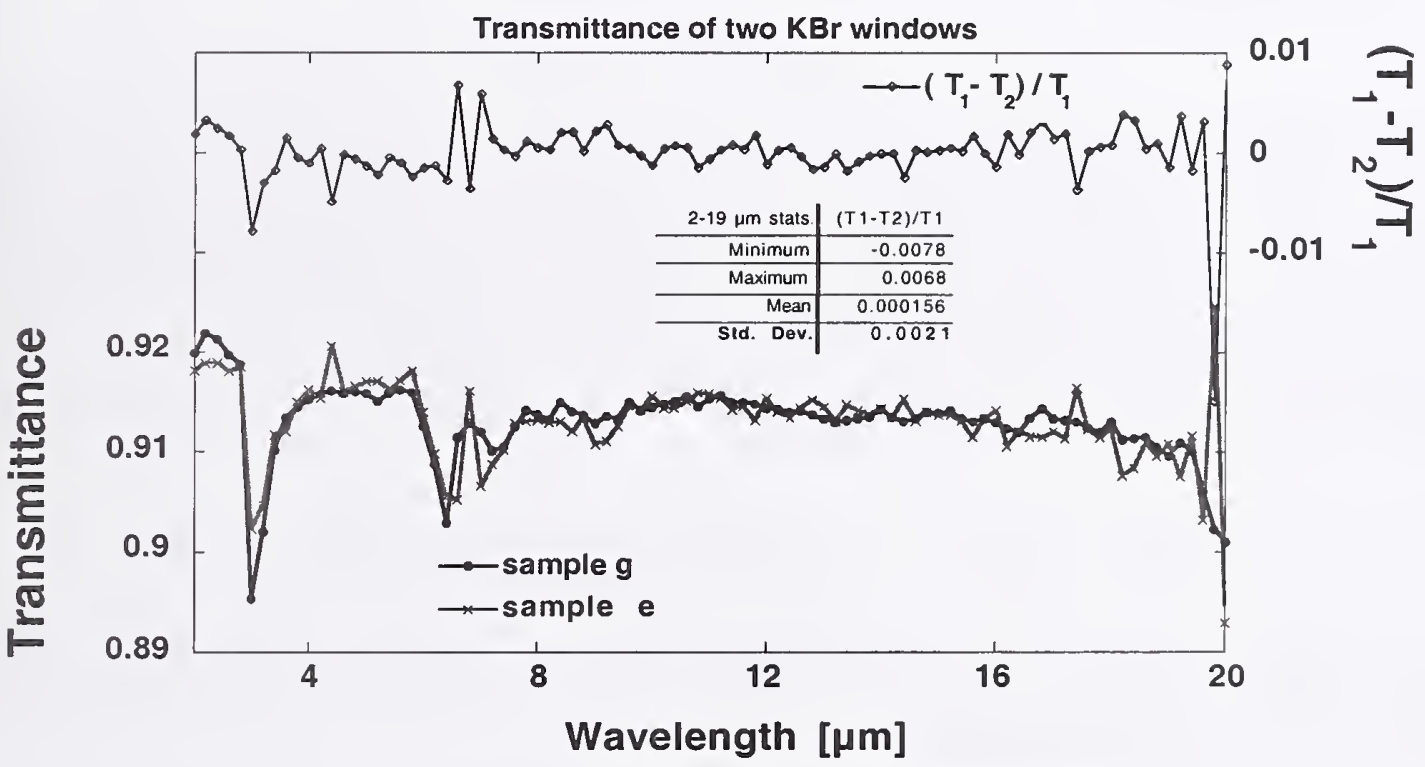

(b)

Figure 6.2 a) Spectral transmittance of a single $\mathrm{KBr}$ window as measured by IR SCF. The mean error bar is $\approx 0.1 \%$ for the region below $16 \mu \mathrm{m}$. b) Spectral transmittance of two $\mathrm{KBr}$ windows and their relative differences.

Figure 6.3 shows the transmittance with associated standard deviation of a single sample of $\mathrm{KBr}$ measured from $0.6 \mu \mathrm{m}$ to $1.8 \mu \mathrm{m}$ by the Vis/NIR SCF. The standard deviation is used as the measurement uncertainty. This measurement was included because the responsivity remains flat there, and it is frequently convenient to be able to use the bolometer for measurements in this range. 


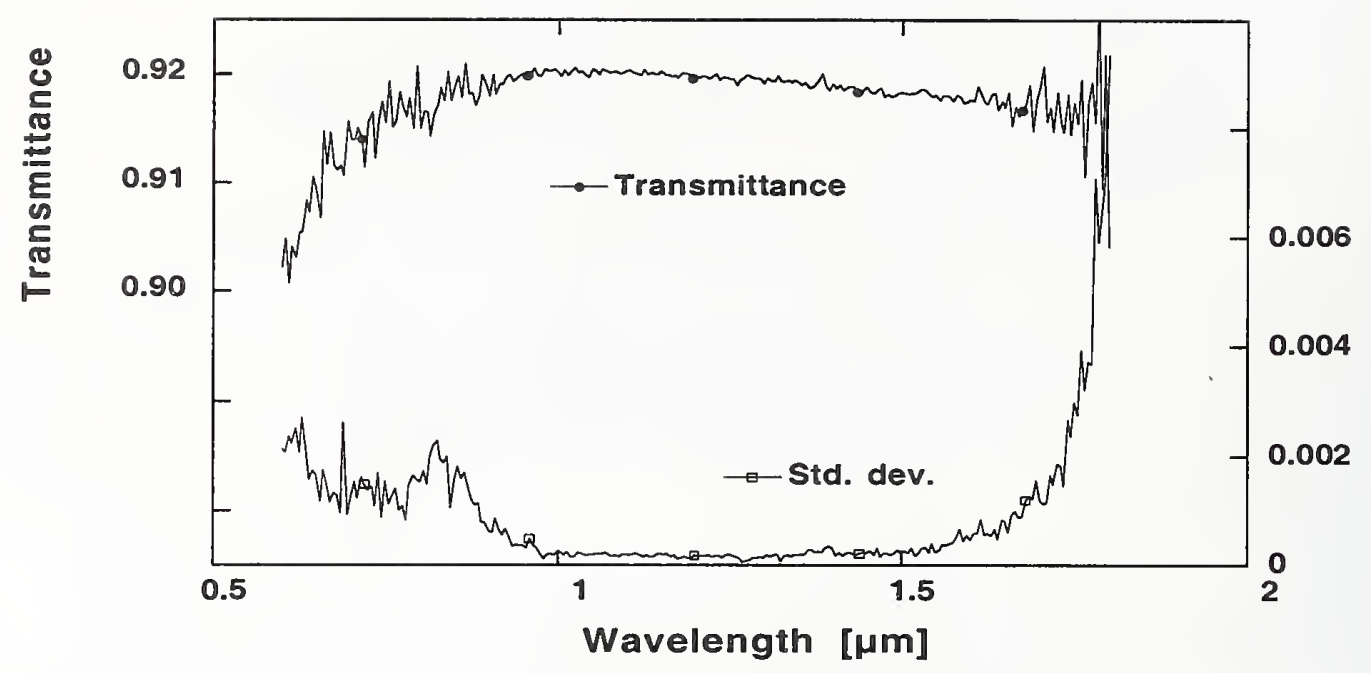

Figure 6.3 The transmittance of a single $\mathrm{KBr}$ window as measured by the Vis/NIR SCF. The lower curve is the standard deviation of the transmittance measurements.

For the window transmission measurements made by the FTIR spectrometer, an arrangement was made to avoid deflecting the transmitted beam. This was done by measuring the transmission of two windows mounted together so that their wedge angles canceled out. The transmission of a single piece was then just the square root of the combined transmission. These FTIR results, although less reproducible than the IR SCF measurements agreed with those results to within $0.2 \%$ to $0.6 \%$. Because the FTIR is more susceptible to drifts than the IR SCF measurements and because of the FTIR can be sensitive to alignment in a way that is difficult to quantify, we used the IR SCF measurements.

An attempt was made to determine the $\mathrm{KBr}$ transmittance by measuring just the reflected beams from the front and back window surfaces. This would have had the advantage of allowing window transmittance to be measured while in place on the dewar. Unfortunately the method yielded inconsistent results and was not pursued. It was suspected that the problem was due to surface scatter or surface absorptance.

\subsubsection{Relative Spectral Responsivity}

The relative spectral responsivity of the bolometer, as shown in Figure 6.4, is just the product of the window transmittance and the bolometer gold black absorptance. Except for a pair of $1 \%$ to $2 \%$ dips (most likely due to window absorption lines) the bolometer responsivity is quite flat. The uncertainty for the $1.8 \mu \mathrm{m}$ to $20 \mu \mathrm{m}$ region is $0.36 \%$, the quadrature sum of the $0.2 \% \mathrm{KBr}$ transmittance uncertainty and the $0.3 \%$ gold black absorptance uncertainty. In the $0.6 \mu \mathrm{m}$ to $2 \mu \mathrm{m}$ region the transmittance uncertainty is so small that the gold black absorptance uncertainty dominates the total uncertainty. 


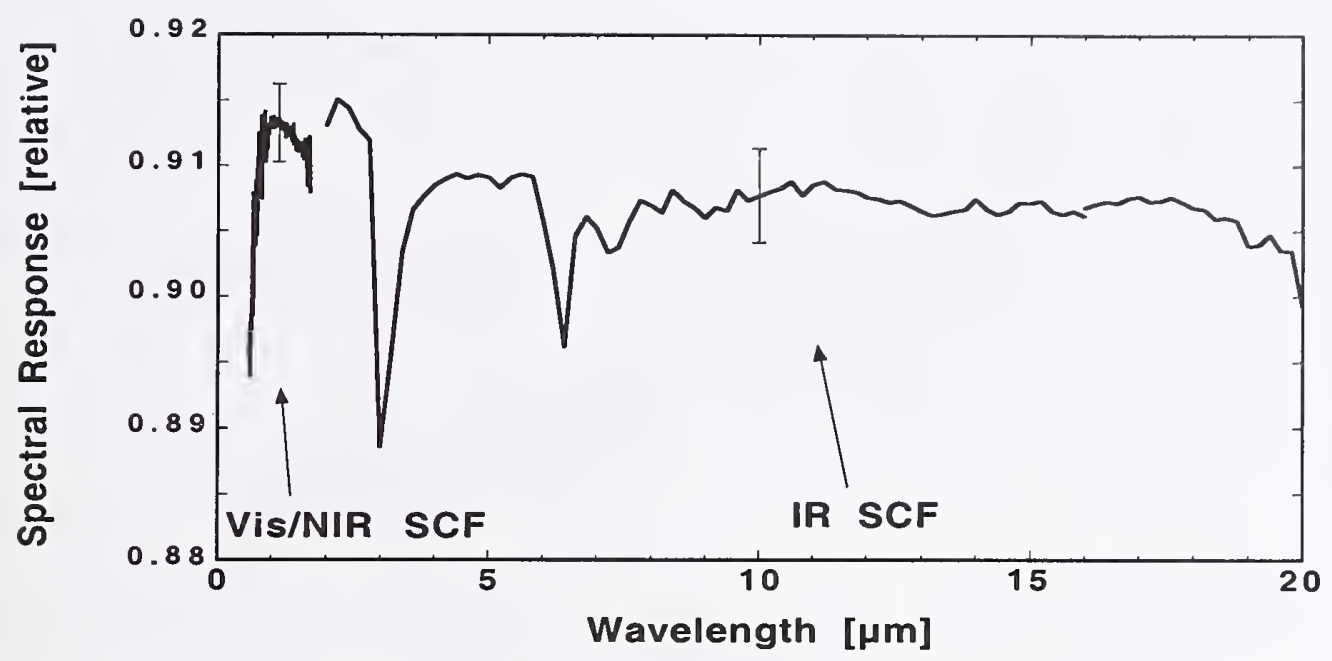

Figure 6.4 Relative spectral responsivity of the bolometer with uncertainties typical of the Vis/NIR and IR spectral regions shown.

\subsubsection{Absolute Responsivity Tie Points}

To convert the relative spectral curve just discussed into an absolute scale, it is necessary to determine the absolute responsivity at one, and preferably, several wavelengths. This was done by comparison to $\mathrm{Si}, \mathrm{Ge}$, and pyroelectric standard detectors which were themselves tied to the HACR. The comparisons were made at specific wavelengths using laser sources at $0.633 \mu \mathrm{m}$, $1.3 \mu \mathrm{m}$ and $10.6 \mu \mathrm{m}$ and over a continuous range from $600 \mathrm{~nm}$ to $900 \mathrm{~nm}$ using the quartz lamp and IR SCF itself as the source. Even though the Vis/NIR calibrations are outside of the intended range of the IR SCF, those measurements are important because of their accuracy and for verification of the spectral flatness of the bolometer.

\subsubsection{IR: Pyroelectric Detector}

The bolometer was compared to a pyroelectric transfer detector over a continuous spectral range from $6 \mu \mathrm{m}$ to $11 \mu \mathrm{m}$ using the IR SCF. This detector was calibrated against the HACR at a single wavelength point of $10.6 \mu \mathrm{m}$ using a laser source [GHE97]. To extend the calibrated spectral range of this transfer detector, a responsivity comparison was made between it and a second pyroelectric detector that did not have an absolute calibration but had a known relative spectral responsivity. This comparison was performed on the IR SCF. Because it was windowless, the relative responsivity of this second detector was found by measuring the total reflectance of its absorptive coating in the infrared using an FTIR spectrometer. This procedure yielded the spectral responsivity of the pyroelectric transfer detector as shown in Figure 6.5. The overall decrease in responsivity with increasing wavelength of the pyroelectric detector is due to decreasing absorptance. 


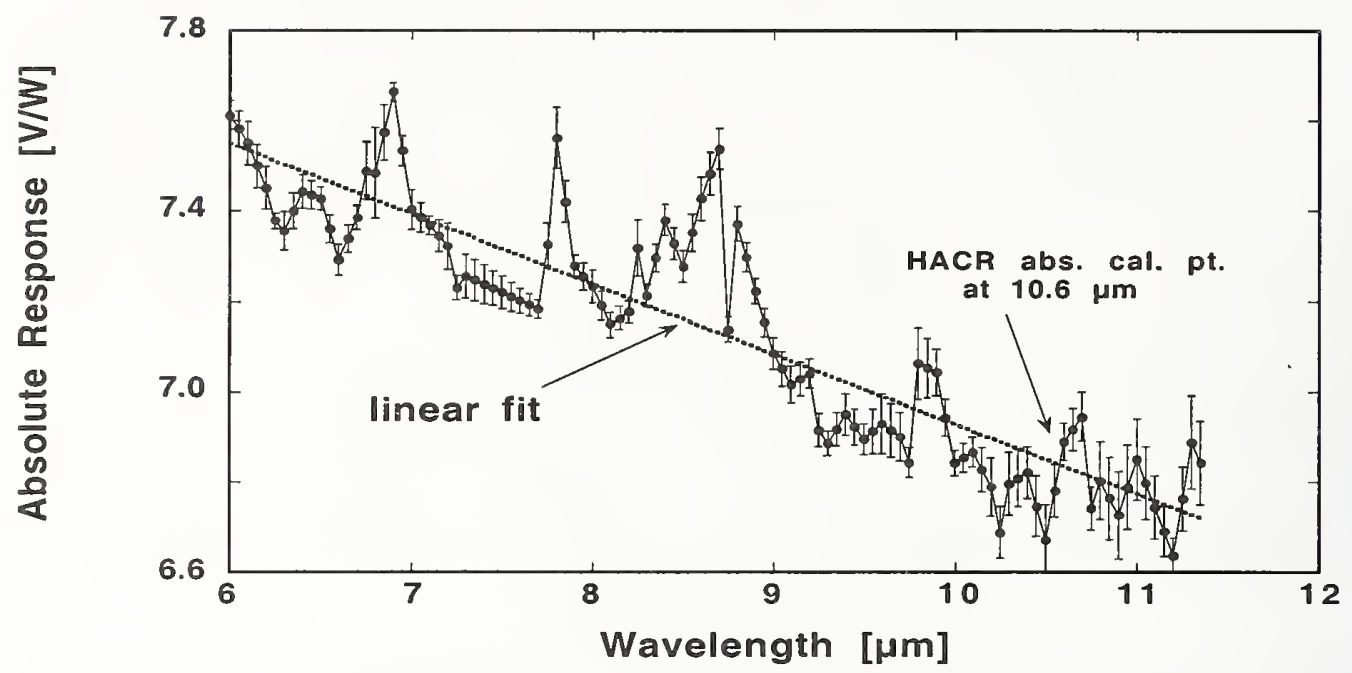

Figure 6.5 The radiant power responsivity of the pyroelectric detector used for scale transfer from the HACR to the bolometer.

The comparison of the bolometer to the pyroelectric transfer detector determined the bolometer responsivity over the $6 \mu \mathrm{m}$ to $11 \mu \mathrm{m}$ spectral range as shown in Figure 6.6. This result combines three comparisons done over different wavelength ranges. Table 6.1 shows the typical component uncertainties associated with this bolometer responsivity calibration. The top portion of the table contains the uncertainties associated with the bolometer responsivity corrections and signal handling. As indicated by the subtotals attributed to each detector, the pyroelectric detector uncertainty dominates the uncertainty of this comparison. The lower portion of the table contains the uncertainties associated with the pyroelectric measurements and pyroelectric responsivity scale. The $\lambda$ uncertainty component is due to the wavelength uncertainty of the monochromator combined with slope of the pyroelectric detector responsivity versus wavelength. The final total relative standard uncertainty for the bolometer responsivity contains all the listed components combined in quadrature with the pyroelectric responsivity scale uncertainty dominating the result. The pyroelectric responsivity scale uncertainty is flat to $1 \%$ from $6 \mu \mathrm{m}$ to $9 \mu \mathrm{m}$ and then smoothly increases to $\approx 1.6 \%$ at $11 \mu \mathrm{m}$. 


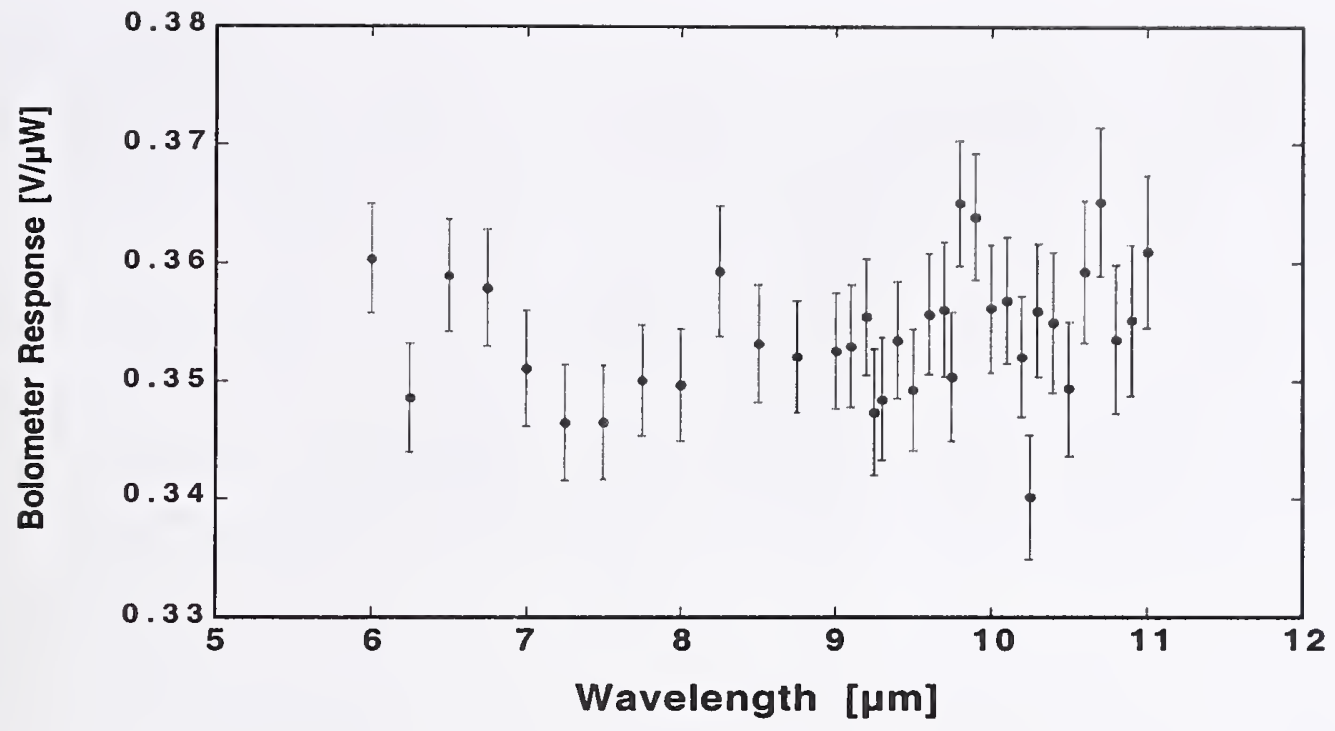

Figure 6.6 Bolometer spectral radiant power responsivity as transferred using the pyroelectric detector.

Table 6.1 Bolometer spectral radiant power responsivity uncertainty budget as transferred from the pyroelectric detector. The last column indicates whether the uncertainty is constant over the spectral range or varies with wavelength.

\begin{tabular}{|c|c|c|c|}
\hline Pyroelectric comparison & \multicolumn{2}{|c|}{$6 \mu \mathrm{m}$ to $11 \mu \mathrm{m}$} & \\
\hline $\begin{array}{l}\text { correction factor/ } \\
\text { uncertainty sources }\end{array}$ & $C$ & $\Delta C / C^{[\%]}$ & \\
\hline$C_{f_{3 \mathrm{~dB}}}(f)$ & 1.1325 & 0.29 & varies \\
\hline$C_{V_{\mathrm{d}}}$ & 1.3611 & 0.19 & varies \\
\hline$V_{\text {bias }}$ & 1.0189 & 0.10 & varies \\
\hline$G_{20 \rightarrow 100}$ & 4.9669 & 0.18 & fixed \\
\hline$C_{\mathrm{NL}}$ & 1.0482 & 0.27 & varies \\
\hline$C_{\text {lockin-output-gain }}$ & 0.9865 & 0.55 & fixed \\
\hline$C_{\text {lockin-gain }}\left(G_{\text {lockin }}=0.1 \mathrm{mV}\right)$ & 1.0032 & 0.21 & fixed \\
\hline $\begin{array}{l}\text { Bolometer signal noise } \\
\text { Bolometer spatial responsivity }\end{array}$ & & $\begin{array}{l}0.05 \\
0.3\end{array}$ & $\begin{array}{l}\text { varies } \\
\text { fixed }\end{array}$ \\
\hline Bolometer Total & & 0.79 & \\
\hline Pyroelectric scale & & $\begin{array}{l}1.18 \\
\text { @10um }\end{array}$ & varies \\
\hline$\lambda$ uncert $(1.5 \mathrm{~nm})$ & & 0.02 & varies \\
\hline Pyroelectric signal & & 0.61 & varies \\
\hline Pyroelectric Total & & 1.33 & varies \\
\hline I Rel. Std. L & & 1.51 & \\
\hline
\end{tabular}

\subsubsection{NIR: Ge Detector}

The bolometer was compared to a Ge transfer detector over a continuous spectral range from $0.9 \mu \mathrm{m}$ to $1.7 \mu \mathrm{m}$ using the IR SCF. (These Ge measurements, as well as the Si measurements described in the next section, required replacing the usual IR gratings in the monochromator with 
a $600 \mathrm{~g} / \mathrm{mm}$ grating.) This transfer detector is tied to the HACR from $0.8 \mu \mathrm{m}$ to $1.8 \mu \mathrm{m}$ via a calibration chain that includes single element and trap configuration Si detectors and a pyroelectric detector and the Vis/NIR SCF [LBP98]. The resulting bolometer responsivity is shown in Figure 6.7b. The curve showing the comparison from $0.9 \mu \mathrm{m}$ to $1.8 \mu \mathrm{m}$ was done at a gain chosen to avoid saturation at the peak Ge signal. The second comparison from $1.5 \mu \mathrm{m}$ to $1.8 \mu \mathrm{m}$ was done with 5 times larger gain, although the two curves show little significant differences. Typical values for the component uncertainties are shown in the Table 6.2 with the total being the quadrature sum. The uncertainties associated with the bolometer are grouped in the first section of the table and those associated with the Ge measurement and Ge responsivity scale are shown in the second group. The final total uncertainty for the bolometer responsivity contains all the listed components combined in quadrature with the Ge responsivity scale uncertainty dominating the result. The Ge responsivity scale uncertainty ranges from slightly less than $1 \%$ from $0.8 \mu \mathrm{m}$ to $1.1 \mu \mathrm{m}$ and then smoothly rises to $\approx 3 \%$ at $1.7 \mu \mathrm{m}$.

In addition to the continuous NIR spectral calibration made using the IR SCF, the absolute bolometer responsivity was measured at $1.31 \mu \mathrm{m}$ by comparison to the Ge standard using a diode laser source. The comparison was performed by repeatedly swapping the bolometer and the Ge detector into and out of the laser beam. The laser power as seen by the Ge detector was stable to $\pm 0.5 \%$ during this comparison. This comparison yielded an absolute bolometer responsivity of $0.3565 \mathrm{~V} / \mu \mathrm{W}$, with the uncertainty given in Table 6.2. From the subtotals it can be seen that it was the uncertainty of the Ge detector that dominates uncertainty of the bolometer-Ge comparison.
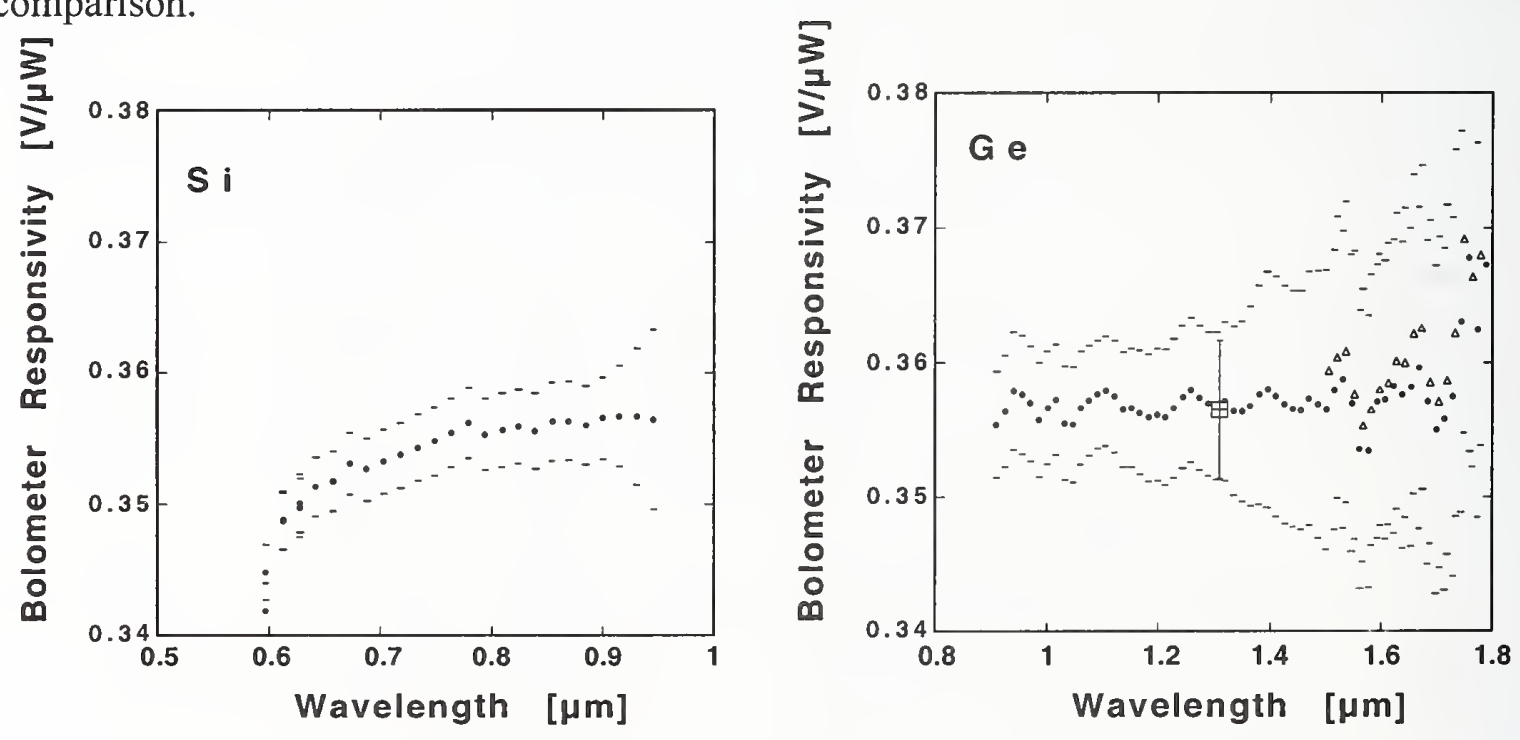

Figure 6.7 Bolometer responsivity calibration by comparison to a) $\mathrm{Si}$ and b) Ge transfer standard detectors. Only the error bar caps are shown for clarity except for the Ge comparison point made using the $1.31 \mu \mathrm{m}$ laser as a source instead of the IR SCF.

Table 6.2 Bolometer spectral radiant power responsivity uncertainty budget as transferred from the Ge detector. The last column indicates whether the uncertainty is constant over the spectral range or varies with wavelength. 


\begin{tabular}{|c|c|c|c|c|c|c|c|}
\hline Ge comparison & \multicolumn{2}{|c|}{$0.9 \mu \mathrm{m}$ to $1.8 \mu \mathrm{m}$} & \multicolumn{2}{|c|}{$1.5 \mu \mathrm{m}$ to $1.8 \mu \mathrm{m}$} & \multicolumn{2}{|c|}{$1.31 \mu \mathrm{m}$} & \\
\hline $\begin{array}{l}\text { correction factor/ } \\
\text { uncertainty sources }\end{array}$ & $C$ & $\Delta C / C^{[\%]}$ & $C$ & $\Delta C / C^{[\%]}$ & C & $\Delta C / C^{[\%]}$ & \\
\hline$C_{f_{3 \mathrm{~dB}}}(f)$ & 1.1014 & 0.05 & 1.1134 & 0.10 & 1.1184 & 0.05 & varies \\
\hline$C_{V_{\mathrm{d}}}$ & 1.3959 & 0.47 & 1.3987 & 0.18 & 1.4807 & 0.07 & varies \\
\hline$V_{\text {bias }}$ & 1.0189 & 0.03 & 1.0197 & 0.01 & 1.0122 & 0.01 & varies \\
\hline$G_{X \rightarrow 100}$ & $\begin{array}{l}0.4930 \\
X=200\end{array}$ & 0.03 & $\begin{array}{l}0.4930 \\
X=200\end{array}$ & 0.03 & $\begin{array}{c}1 \\
X=100\end{array}$ & 0 & fixed \\
\hline$C_{\mathrm{NL}}$ & 1.0004 & 0.00 & 1.0026 & 0.02 & 1.0042 & 0.03 & varies \\
\hline$C_{\text {lockin-outout-gain }}$ & 0.9865 & 0.55 & 0.9865 & 0.55 & 0.9908 & 0.55 & fixed \\
\hline$C_{\text {lockin-gain }}\left(G_{\text {lockin }}\right)$ & 1 & 0 & 1 & 0 & 1 & 0 & fixed \\
\hline Bolometer signal noise & & 0.03 & & 0.02 & & 0.03 & varies \\
\hline Bolometer spatial responsivity & & 0.3 & & 0.3 & & 0.3 & fixed \\
\hline Bolometer Total & & 0.79 & & 0.66 & & 0.63 & \\
\hline $\begin{array}{r}\text { Ge scale } \\
\lambda \text { uncert }(1.5 \mathrm{~nm}) \\
\text { Ge signal }\end{array}$ & & $\begin{array}{c}1.30 \\
@ 1.3 \mu \mathrm{m} \\
0.16 \\
@ 1.3 \mu \mathrm{m} \\
0.14\end{array}$ & & $\begin{array}{c}2.83 \\
@ 1.5 \mu \mathrm{m} \\
0.06 \\
@ 1.5 \mu \mathrm{m} \\
0.00\end{array}$ & & $\begin{array}{c}1.30 \\
@ 1.3 \mu \mathrm{m} \\
0.16 \\
@ 1.3 \mu \mathrm{m} \\
0.19\end{array}$ & $\begin{array}{l}\text { varies } \\
\text { varies } \\
\text { varies }\end{array}$ \\
\hline Ge Total & & 1.32 & & 2.83 & & 1.32 & \\
\hline Grand Total Rel. Std. Uncert. & & 1.56 & & 2.93 & & 1.47 & \\
\hline
\end{tabular}


Table 6.3 Bolometer spectral radiant power responsivity uncertainty budget as transferred from the Si detector. The last column indicates whether the uncertainty is constant over the spectral range or varies with wavelength.

\begin{tabular}{r|cc|c}
\multicolumn{1}{c|}{ Si comparison } & $0.6 \mu \mathrm{m}$ to $0.95 \mu \mathrm{m}$ & \\
\hline \multicolumn{1}{c|}{$\begin{array}{r}\text { correction factor/ } \\
\text { uncertainty sources }\end{array}$} & $C$ & $\Delta C / C[\%]$ & \\
\hline$C_{f_{\text {dda }}}(f)$ & 1.1062 & 0.10 & varies \\
$C_{V_{d}}$ & 1.4024 & 0.19 & varies \\
$V_{\text {bias }}$ & 1.0194 & 0.11 & varies \\
$G_{200 \rightarrow 100}$ & 0.4930 & 0.03 & fixed \\
$C_{\mathrm{NL}}$ & 1.0000 & 0.00 & varies \\
$C_{\text {lockin-ouput-gain }}$ & 0.9865 & 0.55 & fixed \\
$C_{\text {lockin-gain }}\left(G_{\text {lockin }}\right)$ & 1 & 0 & fixed \\
Bolometer signal noise & & 0.06 & varies \\
Bolometer spatial response & & 0.3 & fixed \\
\hline Bolometer Total & & 0.68 & \\
\hline Si scale & & 0.11 & fixed \\
$\lambda$ uncert $(1.5$ nm) & & 0.19 & varies \\
Si signal & & $0.8 \mu \mathrm{m}$ & varies \\
\hline Si Total & & 0.06 & \\
\hline Grand Total Rel. Std. Uncert. & & 0.23 & \\
\hline
\end{tabular}

\subsubsection{Vis: Si Detectors}

For added redundancy, and because the bolometer responsivity is flat down to $\approx 0.6 \mu \mathrm{m}$, the bolometer responsivity was compared to a Si photodiode from $0.6 \mu \mathrm{m}$ to $0.95 \mu \mathrm{m}$ with the results shown in Figure 6.7a. This transfer detector is tied to the HACR from $0.4 \mu \mathrm{m}$ to $0.9 \mu \mathrm{m}$ via a calibration chain that includes single element and trap configuration Si detectors and the Vis/NIR SCF [LBC96, LBP98]. The standard uncertainties of this comparison are given in Table 6.3. For this comparison, the bolometer uncertainty dominates the overall result with the lock-in gain calibration uncertainty being the largest component. This differs from the Ge and pyroelectric comparisons where the transfer detector scale uncertainties dominated.

\subsubsection{Overall Bolometer Absolute Spectral Calibration and Scale Uncertainty}

The overall bolometer spectral responsivity is shown in Figure 6.8 overlaid on the predicted responsivity calculated from the bolometer reflectance and window transmittance. The prediction, being just a relative spectral curve, requires a single scale factor to fit all of the absolute measurements. The predicted curve shown is $T \cdot(1-\mathrm{R}) \cdot 0.3913 \mathrm{~V} / \mu \mathrm{W}$. As can be seen from the figure, the predicted responsivity is a good fit to well within the uncertainties. For the Vis/NIR region, the data shows excellent agreement reflecting the quality of the Si and $\mathrm{Ge}$ transfer standards. The IR region shows much higher variation due to the poorer quality of the pyroelectric transfer detector, which had much lower signal to noise ratio, poorer spatial 
responsivity uniformity, and involved a more difficult calibration transfer. The overall good fit of the predicted curve shape to the actual measurements over the $0.6 \mu \mathrm{m}$ to $11 \mu \mathrm{m}$ range indicates good self-consistency. This should be emphasized as the overall data set includes three independent transfer detectors and a continuous spectral source as well as a laser source. This good self-consistency increases confidence in the regions where absolute measurements were not made and the relative responsivity predictions must be relied upon.

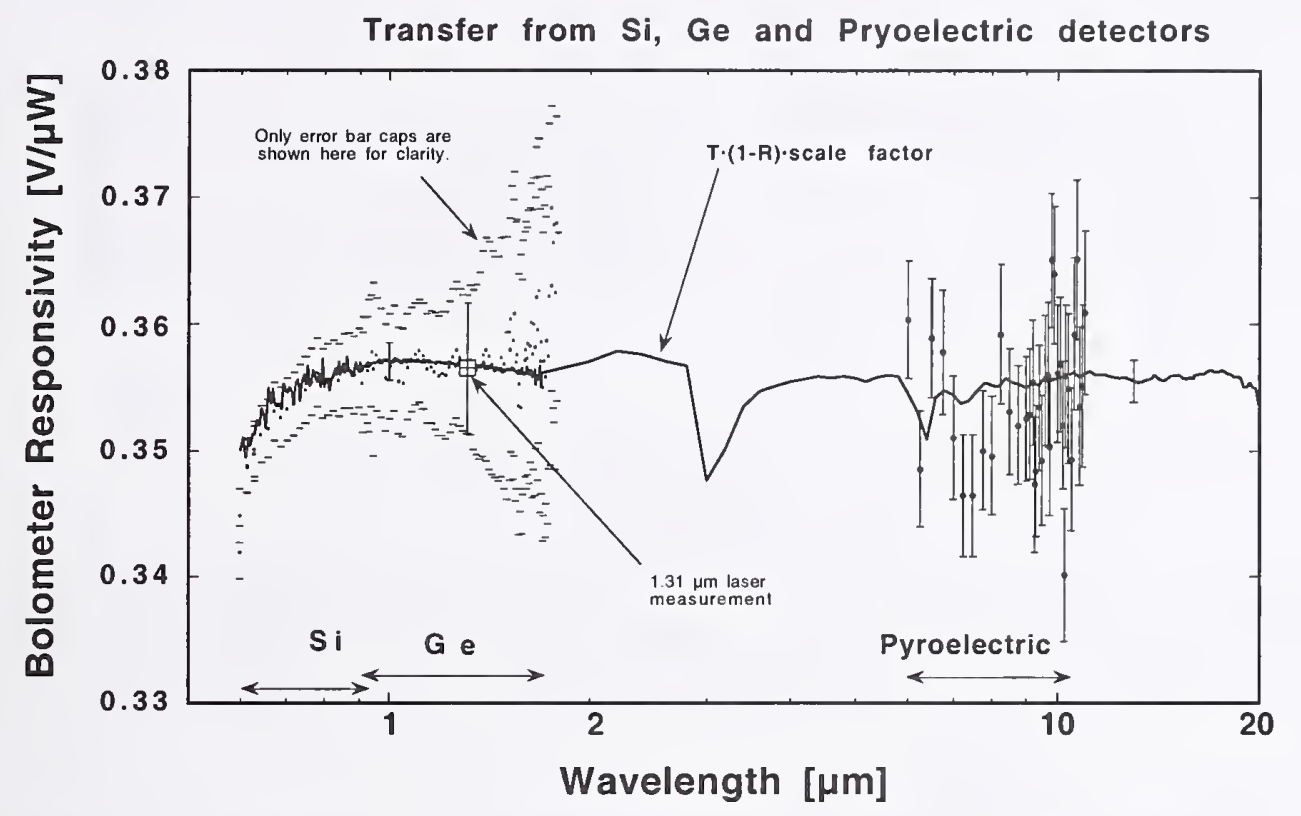

Figure 6.8 The radiant power responsivity of the bolometer in the spectral regions tied to the $\mathrm{Si}$ and $\mathrm{Ge}$ transfer standard detectors are indicated by the solid points below $1.8 \mu \mathrm{m}$. For clarity, only the error bars caps are given for these points. The responsivity measurement made using the laser at $1.31 \mu \mathrm{m}$ is indicated by the box and complete error bar. The bolometer responsivity tied to the pyroelectric transfer detector is indicated by the points between $6 \mu \mathrm{m}$ and $11 \mu \mathrm{m}$ with complete error bars. The solid curve is the $T \cdot(1-\mathrm{R})$ relative responsivity scaled by a factor to give the best overall fit. The two uncertainty error bars for the $\mathrm{T} \cdot(1-\mathrm{R})$ curve shown at $1 \mu \mathrm{m}$ and $12 \mu \mathrm{m}$ are due to the uncertainty of the relative spectral data and the spatial responsivity uncertainty of the bolometer.

The overall scale factor of the relative responsivity curve was determined by taking the ratio of bolometer responsivity to predicted relative responsivity $(T \cdot(1-R))$. The weighted mean of all the ratios was $0.3913 \pm 0.00015$. (The combined standard uncertainty is the standard deviation of the mean.) The weights used were the ratio uncertainty (the quadrature sum of the uncertainties of the responsivity measurements and the responsivity curve.) This constant is the scale factor to convert the relative responsivity curve to an absolute result. All uncertainty components were included, so the nonstatistical uncertainty correlations are ignored here. This analysis assumes a single scale factor over the entire wavelength range. To test this, the ratios of the responsivity measurements to the scaled predicted relative responsivity were plotted versus wavelength and 
fit to a line. As shown in Figure 6.9, the fit stays within $\pm 0.25 \%$ of unity indicating that any systematic tilting of the relative curve is less than this value.

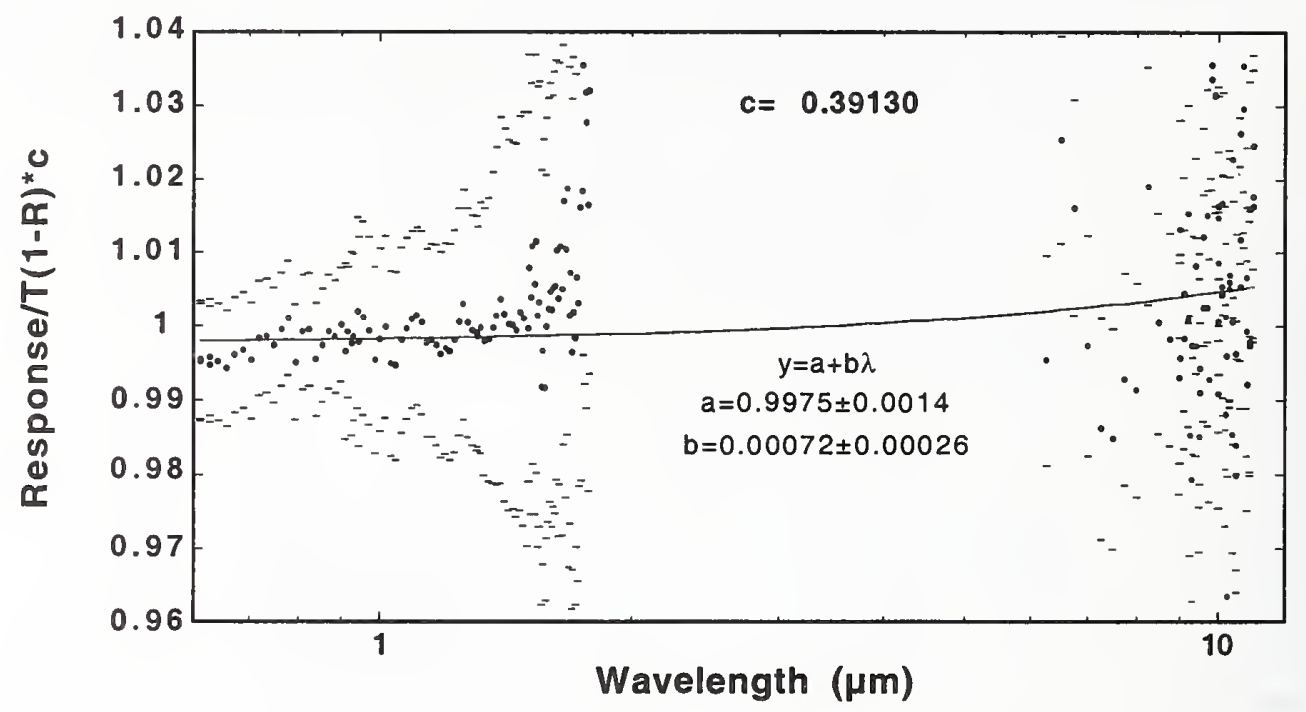

Figure 6.9 Ratio of the absolute bolometer radiant power responsivity to the scaled relative responsivity. The error bars indicate the total uncertainty of the ratios, only the error bar caps are shown for clarity. The solid line is the fit to a line.

To test the effect of neglecting correlation in the uncertainties, a second analysis was done where individual data sets were averaged separately and the determined individual scale factors plotted versus the mean wavelength of the set. Because an individual data set used only a single transfer standard, uncertainty components due to the transfer standard are likely to be correlated. To test the size of the effect on the determined scale factor two sets of weights were used. One used only the uncertainty components that are statistical while the second used the total uncertainties of each point. The resulting scale factor of each data set are shown in Figure 6.10. The differences between the two methods are $\pm 0.25 \%$ or less. The fits of these scale factors versus wavelength are similar to the result of treating all of the data points as a single set.

Taken together, these different analysis schemes support an overall combined standard uncertainty of $\pm 0.25 \%$ in the determined absolute responsivity scale given by the relative responsivity scaled by a factor of 0.3913 , shown as the solid curve in Figure 6.8. This uncertainty along with the combined standard uncertainty associated with a bolometer radiant power measurement at a specific wavelength $(\approx 0.75 \%$ from Table 6.1 to 6.3$)$ gives an overall bolometer radiant power scale combined standard uncertainty of $\approx 0.8 \%$ or expanded uncertainty of $1.6 \%$. This is the uncertainty that can be expected for a bolometer measurement of radiant power made at a single wavelength within the $1.8 \mu \mathrm{m}$ to $20 \mu \mathrm{m}$ range of the IR SCF (assuming adequate measurement time is allowed so as not to be limited by bolometer signal noise). 

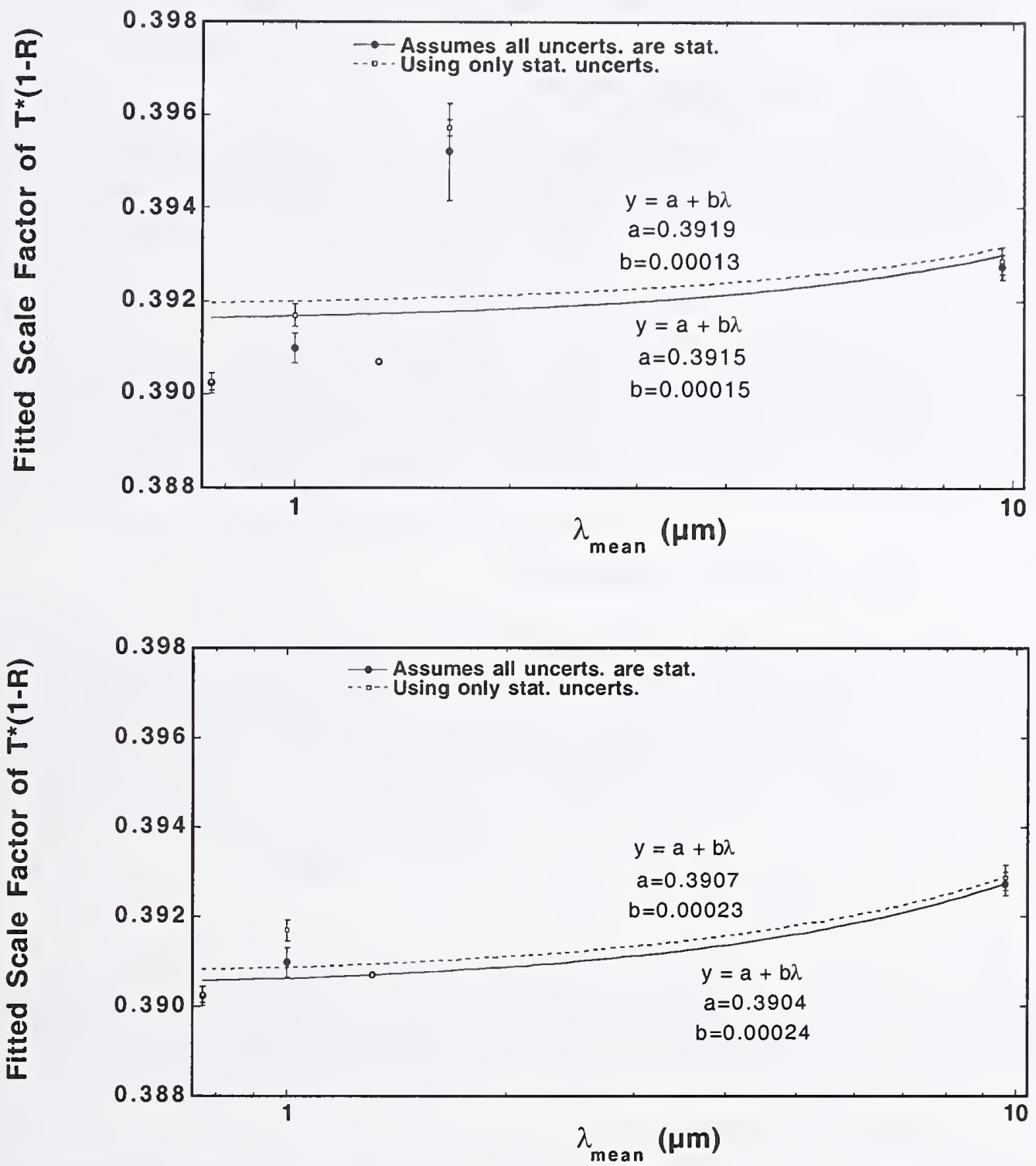

Figure 6.10 Individual data sets used to determine the scale factor of the relative responsivity curve. The solid points indicate all uncertainty components were assumed statistical. For the open points only the statistical uncertainty components were used in the weights for the fits. The solid and dashed lines indicate the fit to the solid and open points respectively. All data sets are shown in a), while the noisy $1.5 \mu \mathrm{m}$ to $1.7 \mu \mathrm{m}$ data set was removed from $\mathrm{b}$ ).

\subsection{InSb Working Standard Spectral Responsivity}

The spectral responsivity and associated uncertainties of the InSb detectors is currently being determined. Upon completion, these detectors will become the working standards for the $2 \mu \mathrm{m}$ to $5.5 \mu \mathrm{m}$ spectral range. 


\section{Operational Procedures and Computer Control}

\subsubsection{Data Taking Program}

All of the computer control and data taking is done by a single main program, which has been designed for maximum flexibility. Data may be taken as a function of a number of parameters: time, chopper frequency, monochromator wavelength, and detector position. The program allows for a number of detectors to be sequentially moved into the monochromator beam. Typically 5 measurements are made on one detector before moving on to the next. When the bolometer is measured, the system records $V_{d}$ at intervals selectable by the operator; typically $V_{d}$ is measured on the initial point and then every fifteenth point thereafter. The program requires inputting the number and position of the detectors to be measured, as well as their gains and the appropriate settings for the lock-in amplifiers. These settings are recorded in the header of the output data files.

\subsubsection{Bolometer Preparation}

The general procedures for bolometer operation are given here. Before any measurements, the $\mathrm{KBr}$ window is visually inspected for evidence of fogging which causes optical scatter. The window must be replaced if any scatter is visible. The window must remain covered with the desiccating cover until measurements require its removal. The bolometer is then prepared for operation by pumping and cooling as described in Section 5.5.1.1. During the filling operation, the state of the bolometer is monitored by manually switching the bias voltage control and verifying that the voltage drop across the bolometer is greater than $0.5 \mathrm{~V}$ indicating that the cryogen has properly cooled the bolometer.

After cooling, at least $6 \mathrm{~h}$ must elapse before any radiant power measurements are made. During this time the frequency response is repeatedly measured. The source for this measurement may be the $633 \mathrm{~nm}$ or $3.39 \mu \mathrm{m} \mathrm{HeNe}$ or the IR SCF operated with a quartz lamp or an arc source, as long as the resulting signal to noise allows for good fit results. The frequency scans typically consist of 35 measurements from $10 \mathrm{~Hz}$ to $300 \mathrm{~Hz}$. During the frequency scans, it is useful to set the data taking program to record the bolometer $V_{d}$ about every 15 points to verify that the bolometer is approaching equilibrium. Each frequency scan is individually fit to the single time constant function of eq (7). The change of the $3 \mathrm{~dB}$ frequency fit results are then in turn fit versus time as shown in Figure 5.5. The $3 \mathrm{~dB}$ point must be between $70 \mathrm{~Hz}$ and $90 \mathrm{~Hz}$ with a rate of change less than $0.3 \mathrm{~Hz} / \mathrm{h}$ before proceeding.

The bolometer is aligned to be on the optical axis of the IR SCF, as described in sec. 4.3.1. The bolometer angle is adjusted to center the input beam on the input window and center the image of the monochromator output aperture on the $3.5 \mathrm{~mm}$ bolometer aperture. Scans of the horizontal and vertical bolometer position are made. As in Figure 5.8, a flat region (to within $\pm 1 \%$ ) of at least $2 \mathrm{~mm}$ indicates proper positional and angular alignment.

\subsubsection{Detector-under-test Preparation}

The setup procedure for the detector-under-test (DUT) will vary depending on its particular characteristics, while the alignment will follow the same procedure performed for the bolometer. 


\subsubsection{Bolometer - DUT Comparison Procedure (Absolute and Relative Responsivity Measurements)}

After alignment of the bolometer and DUT, the proper settings of the detector position and gain parameters can be entered into the program. The typical procedure for comparing the bolometer and DUT versus wavelength is to sample each detector 5 times, change to the next detector before changing the wavelength. This ensures that each detector sees an identical input beam.

\subsubsection{Spatial Uniformity}

The spatial responsivity uniformity of the DUT is measured by scanning its horizontal and vertical position in the plane of the image of the exit aperture of the monochromator. The position of this plane is found by observing the width of the transition as the beam moves past the edge of the detector active area. This width also determines the resolution of the spatial measurements. Typically, $1 \mathrm{~mm}$ spot sizes can be achieved.

\subsubsection{InSb Flashing}

When using the InSb detector, care is taken to reduce the effects of "flashing." This is achieved by limiting the incident radiation below $\approx 2 \mu \mathrm{m}$ by employing cut-on filters and/or appropriate source power distributions which have relatively low short wavelength output. The detector should be monitored for a period $\approx 1 \mathrm{~h}$ to verify that flashing effects are below the desired uncertainty.

\section{Future Work}

With the realization of the IR spectral scale, this comparator is entering an operational phase. Initially, all calibrations will be performed as special tests. With time and increasing experience with both the facility and customer needs, new transfer standard detectors, standardized tests and procedures will develop. As this occurs they will be documented in subsequent editions of this document.

\section{Acknowledgments}

The creation of a document of this type requires the help of many individuals who deserve our thanks. In particular, this work was greatly aided by following the outline of SP 250-41 (1998) created by T. Larason for UV-Vis-NIR detector calibrations. Without this previous publication and the direct help of $\mathrm{T}$. Larason many months of additional work would have been required. The calibrations of the transfer detector used in the bolometer scale realization were done by $\mathrm{J}$. Houston and T. Gentile. The calibration of component transmittances and reflectances were done with the help of S. Kaplan, L. Hanssen and Y. Barnes. Discussions with N. Zhang and K. Eberhardt of the NIST Statistical Engineering Division were of great aid in the analysis of the overall system uncertainty. 


\section{References}

BRM95 J. M. Bridges and A. L. Migdall, "Characterization of Argon Arc Source in the Infrared," Metrologia, 32, 625-628 (1995/96).

BR077 J. M. Bridges and W. R. Ott, "Vacuum Ultraviolet Radiometry. 3: The Argon Mini-Arc as a New Secondary Standard of Spectral Radiance," App. Opt. 16, 367-376 (1977).

BUD83 W. Budde, Optical Radiation Measurements, Volume 4: Physical Detectors of Optical Radiation, Academic Press, Inc., Orlando, FL (1983).

CIE84 Determination of the Spectral Responsivity of Optical Radiation Detectors, Publ. 64 (Commission Internationale de L'Éclairage, Paris, 1984). (Currently available through the U.S. National Committee of the CIE, c/o T. M. Lemons, TLA-Lighting Consultants, Inc., 72 Loring Ave., Salem, MA 01970).

EMC93 G. Eppeldauer, A. L. Migdall, and C. L. Cromer, "Characterization of a High Sensitivity Composite Silicon Bolometer," Metrologia 30, 317-320 (1993).

EMC94 G. Eppeldauer, A. L. Migdall, and C. L. Cromer, "A Cryogenic Silicon Resistance Bolometer for Use as an Infrared Transfer Standard Detector," Thermal Phenomena at Molecular and Microscales and in Cryogenic Infrared Detectors, edited by M. Kaviany et al. (ASME HTD-Vol. 277, New York, NY, 1994), pp. 63-67.

EMG95 G. Eppeldauer, A. L. Migdall, T. R. Gentile, and C. L. Cromer, "Absolute Response Calibration of a Transfer Standard Cryogenic Bolometer," Photodetectors and Power Meters II, Proc. SPIE 2550, 36-46 (1995).

GHE97 T. R. Gentile, J. M. Houston, G. Eppeldauer, A. L. Migdall, and C. L. Cromer "Calibration of a Pyroelectric Detector at $10.6 \mu \mathrm{m}$ with the National Institute of Standards and Technology High-Accuracy Cryogenic Radiometer," Appl. Opt., 36, 36143621 (1997).

GWH95 D. Gupta, L. Wang, L. M. Hanssen, J. J. Hsia, and R. U. Datla, Standard Reference Materials: Polystyrene Films for Calibration the Wavelength Scale of Infrared Spectrophotometer- SRM 1921, Natl. Inst. Stand. Technol., Spec. Publ. 260-122 (1995).

HEC87 E. Hecht, Optics, 2nd edition, Addison-Wesley Publishing Company, Reading, MA (1987).

KBO88 J. Z. Klose, J. M. Bridges, and W. R. Ott, "Radiometric Calibrations of Portable Sources in the Vacuum Ultraviolet," J. Res. Natl. Bur. Stand. (U.S.) 93, 21-39 (1988).

KON78 H. J. Kostkowski and F. E. Nicodemus, An Introduction to the Measurement Equation, Chapter 5 in Self-Study Manual on Optical Radiation Measurements: Part I-Concepts, Chapters 4 and 5, F. E. Nicodemus, Editor, Natl. Bur. Stand. (U.S.), Tech. Note 910-2 (1978). 
KOS79 H. J. Kostkowski, The Relative Spectral Responsivity and Slit-Scattering Function of a Spectroradiometer, Chapter 7 in Self-Study Manual on Optical Radiation Measurements: Part I--Concepts, Chapters 7, 8, and 9, F. E. Nicodemus, Editor, Natl. Bur. Stand. (U.S.), Tech. Note 910-4 (1979).

LBC96 T. C Larason, S. S Bruce, and C. L. Cromer, "The NIST High Accuracy Scale for Absolute Specral Response From 406 nm To 920 nm, " J. Res. Natl. Inst. Stand. Technol. 101, 133-140 (1996).

LBP98 T. C. Larason, S. S. Bruce, and A. C Parr, Spectroradioetric Detector Measurements: Part I - Ultraviolet Detectors and Part II - Visible to Near-Infrared Detectors, Natl. Inst. Stand. Technol., Spec. Publ. 250-41 (1998).

NPW93 D. H. Nettleton, T. R. Prior, and T. H. Ward, "Improved Spectral Responsivity Scales at the NPL, $400 \mathrm{~nm}$ to $20 \mu \mathrm{m}$," Metrologia 30, 425-432 (1993).

OHN97 Y. Ohno, Photometric Calibrations, Natl. Inst. Stand. Technol., Spec. Publ. 25037 (1997).

PAR96 A. C. Parr, A National Measurement System for Radiometry, Photomertry, and Pyrometry Based Upon Absolute Detectors, Natl. Inst. Stand. Technol., Tech. Note 1421 (1996).

SHU83 J. B. Shumaker, Introduction to Coherence in Radiometry, Chapter 10 in SelfStudy Manual on Optical Radiation Measurements: Part I-- Concepts, Chapter 10, F. E. Nicodemus, Editor, Natl. Bur. Stand. (U.S.), Tech. Note 910-6 (1983).

TAK94 B. N. Taylor and C. E. Kuyatt, Guidelines for Evaluating and Expressing the Uncertainty of NIST Measurement Results, Natl. Inst. Stand. Technol., Tech. Note 1297 (1994 ed.). 


\section{THE SP 250 SERIES ON NIST MEASUREMENT SERVICES*}

SP 250-1 Spectral Radiance Calibrations PB87179883

SP 250-2 Far Ultraviolet Detector Standards PB87227609

SP 250-3 Radiometric Standards in the Vacuum Ultraviolet PB87227625

SP 250-4 Fricke Dosimetry in High-Energy Electron Beams PB88110374

SP 250-5 Alpha-Particle Calibrations PB88168620

SP 250-6 Regular Spectral Transmittance PB88108550

SP 250-7 Radiance Temperature Calibrations PB88123674

SP 250-8 Spectral Reflectance PB88109905

SP 250-9 Calibration of Beta-Particle-Emitting Ophthalmic Applicators PB88108535

SP 250-10 Radioactivity Calibrations with the " $4 \pi$ " Gamma Ionization Chamber and Other Radioactivity Calibration Capabilities PB88123708

SP 250-11 Dosimetry for High Dose Applications PB88201587

SP 250-12 Neutron Personnel Dosimetry PB87227617

SP 250-13 Activation Foil Irradiation with Californium Fission Sources PB88217443

SP 250-14 Activation Foil Irradiation by Reactor Cavity Fission Sources PB88217435

SP 250-15 Photometric Calibrations PB88153747

SP 250-16 Calibration of X-Ray and Gamma-Ray Measuring Instruments PB88211826

SP 250-17 The NBS Photodetector Spectral Response Calibration Transfer Program PB88201595

SP 250-18 Neutron Source Strength Calibrations PB88211818

SP 250-19 Calibration of Gamma-Ray-Emitting Brachytherapy Sources PB89193858

SP 250-20 Spectral Irradiance Calibrations PB88123781

SP 250-21 Calibration of Beta-Particle Radiation Instrumentation PB88201579

SP 250-22 Platinum Resistance Thermometer Calibrations PB88138367

SP 250-23 Liquid-in-Glass Thermometer Calibration Service PB89128888 SP 250-24 Standard Cell Calibrations
PB88123690

SP 250-25 Calibration Service for Inductive Voltage Dividers

SP 250-26 NBS Phase Angle Calibration Services PB88225636

SP 250-27 AC-DC Difference Calibrations PB892222616

SP 250-28 Solid-State DC Voltage Standard Calibrations PB88168703

SP 250-29 Traceable Frequency Calibrations PB88168364

SP 250-30 GOES Satellite Time Code Dissemination: Description and Operation PB88168760

SP 250-31 Mass Calibrations PB89153894

SP 250-32 A Calibration Service for $30 \mathrm{MHz}$ Attenuation and Phase Shift PB88238324

SP 250-33 A Calibration Service for Voltage Transformers and High-Voltage Capacitors PB882252903

SP 250-34 High Vacuum Standard and Its Use PB89193841

SP 250-35 The Calibration of Thermocouples and Thermocouple Materials PB89209340

SP 250-36 A Calibration Service for Current Transformers PB91216770

SP 250-37 Photometric Calibrations PB97148472

SP 250-38 NIST Leak Calibration Service PB92149772

SP 250-39 NIST Pressure Calibration Service PB94164043

SP 250-40 Absorbed-Dose Calibration of Ionization Chambers in a ${ }^{60} \mathrm{Co}$ Gamma-Ray Beam SN003-003-03034-1 \$2.00

SP 250-41 Spectroradiometric Detector Measurements: Part I - Ultraviolet Detectors and Part II - Visible to Near-Infrared Detectors SN003-003-03550-5 \$9.50

SP 250-43 Radiance Temperature Calibrations SN003-003-03511-4 \$10.00

SP 250-44 Radiation Processing Dosimetry Calibration Services and Measurement Assurance Program SN003-003-03513-1 \$3.75

SP 250-45 Radiation Processing Dosimetry Calibration Services: Manual of Calibration Procedures SN003-003-03514-9 \$4.00

* Entries containing a stock number (SN003-003-) and price can be purchased from the Superintendent of Documents, U.S. Government Printing Office, Washington, DC 20402. GPO will accept checks, money orders, VISA, and MasterCard. For more infomation, or to place an order, call (202) 512-1800. Be sure to cite the stock number on all orders.

Entries containing PB numbers can be purchased from the National Technical Information Service, Springfield, VA 22161. NTIS will accept American Express in addition to the payment methods listed for GPO. For more information call (703)487-4650; to place an order call (800) 553-6487. Fax: (703) 321-8547. Be sure to cite the PB number on all orders.

Entries without stock or PB numbers are in preparation. 
SP 250-46 NIST Multifunction Calibration System SN003-003-03515-7 \$2.75

SP 250-47 NIST Calibration Service for Capacitance Standards at Low Frequencies SN003-003-03549-1 \$7.00

SP 250-48 Spectral Reflectance SN003-003-03545-9 $\$ 14.00$

* Entries containing a stock number (SN003-003-) and price can be purchased from the Superintendent of Documents, U.S. Government Printing Office, Washington, DC 20402. GPO will accept checks, money orders, VISA, and MasterCard. For more infomation, or to place an order, call (202) 512-1800. Be sure to cite the stock number on all orders.

Entries containing PB numbers can be purchased from the National Technical Information Service, Springfield, VA 22161. NTIS will accept American Express in addition to the payment methods listed for GPO. For more information call (703)487-4650; to place an order call (800) 553-6487. Fax: (703) 321-8547. Be sure to cite the PB number on all orders.

Entries without stock or PB numbers are in preparation. 


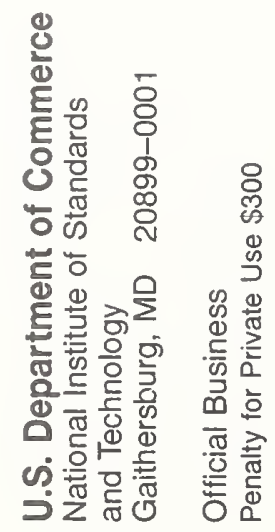

